\title{
First Total Synthesis and Structural Reassignment of (-)-Aplysiallene
}

\author{
Jian Wang and Brian L. Pagenkopf ${ }^{\star}$ \\ Department of Chemistry, University of Western Ontario \\ 1151 Richmond St., London, ON, N6A 5B7, Canada \\ bpagenko@uwo.ca
}

Experimental Details

All reactions were run under an atmosphere of argon unless otherwise indicated. Flasks were oven or flame dried and allowed to cool under argon or in a desiccator prior to use. A solution of anhydrous tert-butylhydroperoxide (TBHP) in isooctane was prepared according to the previously described procedure. ${ }^{1}$ All other reagents and chemicals were obtained from common commercial sources and used without further purification unless otherwise noted.

Tetrahydrofuran (THF), diethyl ether $\left(\mathrm{Et}_{2} \mathrm{O}\right)$, hexanes, dichloromethane $\left(\mathrm{CH}_{2} \mathrm{Cl}_{2}\right)$ were dried by passage through a column of alumina using a solvent dispensing system. Triethylamine $\left(\mathrm{Et}_{3} \mathrm{~N}\right)$ was dried by distillation over $\mathrm{CaH}_{2}$ under nitrogen. Methanol $(\mathrm{MeOH})$ was dried by distillation over $\mathrm{Mg}$ under nitrogen. Progress of reactions was monitored by thin layer chromatography (TLC) performed on EM 250 Kieselgel 60 F254 silica gel plates. The plates were visualized by staining with ceric ammonium molybdate or potassium permanganate $\left(1.5 \mathrm{~g} \mathrm{KMnO}_{4}, 10 \mathrm{~g}\right.$ $\mathrm{K}_{2} \mathrm{CO}_{3}, 1.25 \mathrm{~mL} 10 \%$ aqueous $\mathrm{NaOH}$ in $200 \mathrm{~mL}$ water). Column chromatography was performed with Sorbent Technologies 32-63 $\mu \mathrm{m}$ silica gel according to the method of Still. ${ }^{2}$ The ${ }^{1} \mathrm{H}$ and ${ }^{13} \mathrm{C}$ nuclear magnetic resonance (NMR) spectra were obtained on a Varian Inova 600 MHz NMR spectrometer. Chemical shifts are reported in parts per million (ppm) downfield from tetramethylsilane and are, in all cases, referenced to the residual proton resonance peaks: $\delta 7.26$ for $\mathrm{CDCl}_{3}, 7.16$ for $\mathrm{C}_{6} \mathrm{D}_{6}$. When peak multiplicities are given, the following abbreviations are used: s, singlet; d, doublet; t, triplet; q, quartet; dd, doublet of a doublet; dt, doublet of a triplet; $\mathrm{m}$, multiplet; brs, broad singlet. The ${ }^{13} \mathrm{C}$ NMR chemical shifts were reported in ppm relative to the center peak of the multiplet for deuterated solvents: $\delta 77.0$ (t) for $\mathrm{CDCl}_{3}, 128.0$ (t) for $\mathrm{C}_{6} \mathrm{D}_{6}$. ${ }^{13} \mathrm{C}$ NMR spectra were run with broadband ${ }^{1} \mathrm{H}$ decoupling. Coupling constants for all spectra are reported in Hertz $(\mathrm{Hz})$.

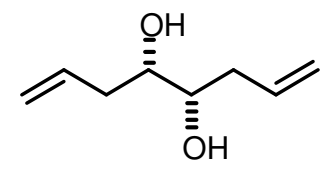

(4S,5S)-Octa-1,7-diene-4,5-diol (ent-5)

To a suspension of $\mathrm{CuBr}(28.0 \mathrm{~g}, 195 \mathrm{mmol}, 1.6$ equiv) in THF $(60 \mathrm{~mL})$ at $-30{ }^{\circ} \mathrm{C}$ is added vinyl magnesium bromide $(0.49 \mathrm{M}$ in THF, $600 \mathrm{~mL}, 294$ mmol, 2.4 equiv) dropwise. After $0.5 \mathrm{~h}$ a solution of (S,S)-diepoxybutane (10.8 g, $125 \mathrm{mmol}, 1.0$ equiv) in THF $(70 \mathrm{~mL})$ is added dropwise. After $4 \mathrm{~h}$ the mixture was allowed to slowly warm to $0{ }^{\circ} \mathrm{C}$ and saturated aqueous $\mathrm{NaHCO}_{3}(150 \mathrm{~mL})$ was added. The 
precipitates were filtered off and extracted with EtOAc in a Soxhlet apparatus, and the filtrate was separated and the aqueous layer was extrated with EtOAc $(3 \times 100 \mathrm{~mL})$. The organic extracts were combined, dried $\left(\mathrm{MgSO}_{4}\right)$, concentrated under reduced pressure and purified by flash chromatography on silica gel (elution gradient: 5\% EtOAc/hexanes to 50\% EtOAc/hexanes) to give the title compound as a white solid $(12.7 \mathrm{~g}, 72 \%)$.

Rf 0.37 (50\% EtOAc/hexanes); mp 42.0 - $44.0{ }^{\circ} \mathrm{C},{ }^{1} \mathrm{H}$ NMR $\left(600 \mathrm{MHz}, \mathrm{CDCl}_{3}\right) \delta 5.85-5.78(\mathrm{~m}$, 2H), 5.12-5.07 (m, 4H), 3.51-3.47 (m, 2H), 2.90-2.89 (m, 2H), 2.33-2.29 (m, 2H), 2.24-2.19 $(\mathrm{m}, 2 \mathrm{H}) ;{ }^{13} \mathrm{C}$ NMR $\left(150 \mathrm{MHz}, \mathrm{CDCl}_{3}\right) \delta 134.4,117.7,72.7,38.0 ;[\alpha]_{\mathrm{D}}=-43.8^{\circ}(\mathrm{c}=0.016$, $\mathrm{EtOH})$.

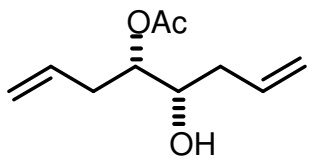

Acetic acid (1S,2S)-1-allyl-2-hydroxypent-4-enyl ester (7)

A solution of ent-5 (2.42 g, $17 \mathrm{mmol}, 1.0$ equiv), triethyl orthoacetate (5.59 $\mathrm{g}, 34.5 \mathrm{mmol}, 2.0$ equiv) and $p$-toluenesulfonic acid monohydrate $(97 \mathrm{mg}$, $0.5 \mathrm{mmol}, 0.03$ equiv) in THF $(10 \mathrm{~mL})$ was allowed to stand at room temperature for $20 \mathrm{~h}$. The mixture was then concentrated under reduced pressure, dissolved in a mixture of $\mathrm{AcOH}(9 \mathrm{~mL})$ and $\mathrm{H}_{2} \mathrm{O}(1.8 \mathrm{~mL})$ and allowed to stand at room temperature for $1 \mathrm{~h}$. The mixture was then concentrated under reduced pressure, mixed with saturated aqueous $\mathrm{NaHCO}_{3}(20 \mathrm{~mL})$, extracted with EtOAc $(3 \times 20 \mathrm{~mL})$, dried $\left(\mathrm{MgSO}_{4}\right)$, concentrated under reduced pressure and purified by flash chromatography on silica gel (33\% EtOAc/hexanes) to give the title compound as a colorless oil (3.12 g, 100\%).

Rf 0.43 (33\% EtOAc/hexanes); ${ }^{1} \mathrm{H}$ NMR (600 MHz, $\left.\mathrm{CDCl}_{3}\right) \delta 5.85-5.72(\mathrm{~m}, 2 \mathrm{H})$, 5.15-5.11 (m, $3 \mathrm{H}), 5.08(\mathrm{dd}, J=10.0,1.2 \mathrm{~Hz}, 1 \mathrm{H}), 4.92-4.89(\mathrm{~m}, 1 \mathrm{H}), 3.73-3.69(\mathrm{~m}, 1 \mathrm{H}), 2.49-2.45(\mathrm{~m}, 1 \mathrm{H})$, 2.42-2.37 (m, 1H), 2.30-2.26 (m, 1H), 2.23-2.18 (m, 1H), $2.09(\mathrm{~s}, 3 \mathrm{H}), 1.83-1.82(\mathrm{br}, 1 \mathrm{H}) ;{ }^{13} \mathrm{C}$ NMR $\left(150 \mathrm{MHz}, \mathrm{CDCl}_{3}\right) \delta 170.6,133.8,133.3,118.4,118.1,74.8,71.0,38.2,35.3,21.0 ;$ HRMS $m / z 185.1173$ (calc'd for $\mathrm{C}_{10} \mathrm{H}_{17} \mathrm{O}_{3}[\mathrm{M}+\mathrm{H}]^{+}, 185.1178$ ).

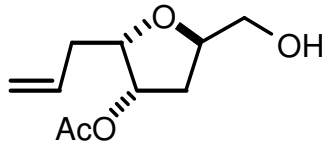

Acetic acid (2S,3S,5R)-2-allyl-5-hydroxymethyltetrahydrofuran-3-yl ester (8)

A solution of TBHP (5.33 $\mathrm{M}$ in isooctane, $1.7 \mathrm{~mL}, 9.2 \mathrm{mmol}, 1.0$ equiv) was added at room temperature to a solution of $7(1.70 \mathrm{~g}, 9.2 \mathrm{mmol}, 1.0$ equiv) and $\mathrm{Co}^{\mathrm{II}}(\mathrm{Modp})^{2}(500 \mathrm{mg}, 0.92 \mathrm{mmol}, 0.1$ equiv) in $i \mathrm{PrOH}(90 \mathrm{~mL})$. The reaction mixture was heated to $50{ }^{\circ} \mathrm{C}$ under 1 atm of $\mathrm{O}_{2}$ (balloon) for $24 \mathrm{~h}$, then concentrated under reduced pressure and purified by flash chromatography on silica gel (elution gradient: 33\% EtOAc/hexanes to $50 \%$ EtOAc/hexanes) to give the title compound as a light green oil (1.47 g, $80 \%)$.

Rf 0.21 (50\% EtOAc/hexanes); ${ }^{1} \mathrm{H}$ NMR (600 MHz, $\left.\mathrm{CDCl}_{3}\right) \delta 5.77$ (dddd, $J=17.0,10.5,8.2$, $7.0 \mathrm{~Hz}, 1 \mathrm{H}), 5.34-5.33(\mathrm{~m}, 1 \mathrm{H}), 5.10$ (dddd, $J=17.0,4.7,1.8,1.8 \mathrm{~Hz}, 1 \mathrm{H}), 5.06-5.05$ (m, 1H), $4.32-4.28(\mathrm{~m}, 1 \mathrm{H}), 4.03(\mathrm{ddd}, J=10.0,7.0,3.5 \mathrm{~Hz}, 1 \mathrm{H}), 3.75-3.73(\mathrm{br}, 1 \mathrm{H}), 3.52-3.48(\mathrm{~m}, 1 \mathrm{H})$, 2.41-2.36 (m, 1H), 2.34-2.29 (m, 1H), 2.10-2.06 (m, 1H), $2.08(\mathrm{~s}, 3 \mathrm{H}), 2.05-2.00(\mathrm{~m}, 2 \mathrm{H}) ;{ }^{13} \mathrm{C}$ NMR $\left(150 \mathrm{MHz}, \mathrm{CDCl}_{3}\right) \delta 170.3,134.1,117.3,80.7,77.8,75.1,64.4,34.5,33.9,21.0$; HRMS $m / z 201.1121$ (calc'd for $\mathrm{C}_{10} \mathrm{H}_{17} \mathrm{O}_{4}[\mathrm{M}+\mathrm{H}]^{+}, 201.1127$ ). 


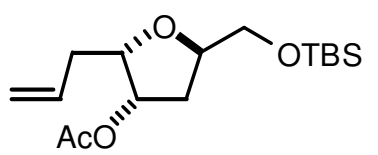

Acetic acid (2S,3S,5R)-2-allyl-5-(tert-

butyldimethylsilanyloxymethyl)-tetrahydrofuran-3-yl ester (17)

A solution of 8 (1.44 g, $7.2 \mathrm{mmol}, 1.0$ equiv), TBSCl (1.35 g, $9 \mathrm{mmol}$, 1.25 equiv) and imidazole ( $1.35 \mathrm{~g}, 20 \mathrm{mmol}, 2.75$ equiv) in DMF $(9 \mathrm{~mL})$ was allowed to stand at room temperature for $15 \mathrm{~h}$. The mixture was then diluted with $\mathrm{Et}_{2} \mathrm{O}$ (50 $\mathrm{mL})$, washed with $\mathrm{H}_{2} \mathrm{O}(50 \mathrm{~mL})$, dried $\left(\mathrm{MgSO}_{4}\right)$, concentrated under reduced pressure and purified by flash chromatography on silica gel (elution gradient: 10\% EtOAc/hexanes to 20\% EtOAc/hexanes) to give the title compound as a colorless oil (2.14 g, 95\%).

$\mathrm{R} f 0.50$ (20\% EtOAc/hexanes); ${ }^{1} \mathrm{H}$ NMR (600 MHz, $\left.\mathrm{CDCl}_{3}\right) \delta 5.81-5.75(\mathrm{~m}, 1 \mathrm{H}), 5.32(\mathrm{t}, J=4.1$ $\mathrm{Hz}, 1 \mathrm{H}), 5.11-5.08(\mathrm{~m}, 1 \mathrm{H}), 5.05-5.03(\mathrm{~m}, 1 \mathrm{H}), 4.27-4.23(\mathrm{~m} 1 \mathrm{H}), 4.02(\mathrm{dt}, J=6.4,3.5 \mathrm{~Hz}$, $1 \mathrm{H}), 3.69$ (dd, $J=10.5,4.1 \mathrm{~Hz}, 1 \mathrm{H}), 3.61$ (ddd, $J=11.7,4.1,1.2 \mathrm{~Hz}, 1 \mathrm{H}), 2.40-2.35(\mathrm{~m}, 1 \mathrm{H})$, $2.33-2.28(\mathrm{~m}, 1 \mathrm{H}), 2.19(\mathrm{ddd}, J=14.1,8.2,5.3 \mathrm{~Hz}, 1 \mathrm{H}), 2.08(\mathrm{~d}, J=1.2 \mathrm{~Hz}, 3 \mathrm{H}), 2.02(\mathrm{dd}, J=$ $14.1,7.0 \mathrm{~Hz}, 1 \mathrm{H}), 0.89$ (s, 9H), $0.05(\mathrm{~s}, 6 \mathrm{H}) ;{ }^{13} \mathrm{C} \mathrm{NMR}\left(150 \mathrm{MHz}, \mathrm{CDCl}_{3}\right) \delta 170.5,134.4,116.9$, 80.8, 77.7, 75.5, 65.4, 34.9, 34.0, 25.9, 21.1, 18.3, -5.3, -5.4; HRMS $m / z 315.1980$ (calc'd for $\left.\mathrm{C}_{16} \mathrm{H}_{31} \mathrm{O}_{4} \mathrm{Si}[\mathrm{M}+\mathrm{H}]^{+}, 315.1992\right)$.

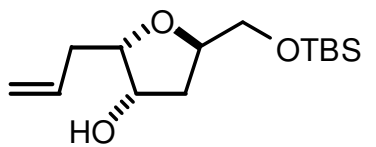

(2S,3S,5R)-2-Allyl-5-(tert-butyldimethylsilanyloxymethyl)tetrahydrofuran-3-ol (18)

A portion of a solution of $\mathrm{EtBr}\left(1.5 \mathrm{~mL}, 20.5 \mathrm{mmol}, 3.0\right.$ equiv) in $\mathrm{Et}_{2} \mathrm{O}$ (7 mL) was added to $\mathrm{Mg}$ (500 $\mathrm{mg}, 20.5 \mathrm{mmol}, 3.0$ equiv) to initiate a gentle reflux, after which the rest of the solution was added dropwise. The mixture was then heated to reflux for $10 \mathrm{~min}$, cooled to room temperature, and slowly added to a solution of $\mathbf{1 7}$ $\left(2.14 \mathrm{~g}, 6.8 \mathrm{mmol}, 1.0\right.$ equiv) in $\mathrm{Et}_{2} \mathrm{O}(7 \mathrm{~mL})$ at $0{ }^{\circ} \mathrm{C}$. The mixture was then allowed to stand at room temperature for $1 \mathrm{~h}$, cooled to $0{ }^{\circ} \mathrm{C}$, and saturated aqueous $\mathrm{NH}_{4} \mathrm{Cl}(20 \mathrm{~mL})$ was added. The layers were separated and the aqueous layer was extracted with EtOAc $(2 \times 20 \mathrm{~mL})$. The organic extracts were combined, dried $\left(\mathrm{MgSO}_{4}\right)$, concentrated under reduced pressure and purified by flash chromatography on silica gel (elution gradient: 20\% EtOAc/hexanes to $33 \%$ EtOAc/hexanes) to give the title compound as a colorless oil (1.83 g, 99\%).

$\mathrm{R} f 0.38$ (33\% EtOAc/hexanes); ${ }^{1} \mathrm{H}$ NMR $\left(600 \mathrm{MHz}, \mathrm{CDCl}_{3}\right) \delta 5.86$ (ddt, $J=17.0,10.5,7.0 \mathrm{~Hz}$, $1 \mathrm{H}), 5.17(\mathrm{dq}, J=17.0,1.8 \mathrm{~Hz}, 1 \mathrm{H}), 5.08(\mathrm{dd}, J=10.0,1.8 \mathrm{~Hz}, 1 \mathrm{H}), 4.30-4.26(\mathrm{~m}, 2 \mathrm{H}), 3.89$ (dt, $J=7.0,2.9 \mathrm{~Hz}, 1 \mathrm{H}), 3.68(\mathrm{dd}, J=10.5,4.1 \mathrm{~Hz}, 1 \mathrm{H}), 3.61(\mathrm{dd}, J=10.5,4.1 \mathrm{~Hz}, 1 \mathrm{H}), 2.48-2.43$ (m, 1H), 2.41-2.36 (m, 1H), 2.10-2.04 (m 1H), 2.01-1.97 (m, 1H), $1.55(\mathrm{~d}, J=5.9 \mathrm{~Hz}, 1 \mathrm{H}), 0.89$ $(\mathrm{s}, 9 \mathrm{H}), 0.06(\mathrm{~s}, 6 \mathrm{H}) ;{ }^{13} \mathrm{C}$ NMR $\left(150 \mathrm{MHz}, \mathrm{CDCl}_{3}\right) \delta 134.8,117.0,82.0,77.4,73.4,65.6,37.1$, 33.8, 25.9, 18.3, -5.3, -5.4; HRMS $m / z, 273.1892$ (calc'd for $\mathrm{C}_{14} \mathrm{H}_{29} \mathrm{O}_{3} \mathrm{Si}[\mathrm{M}+\mathrm{H}]^{+}, 273.1886$ ).

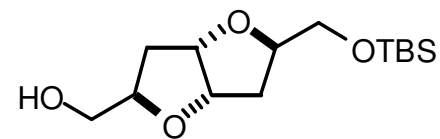

[(2R,3aS,5R,6aS)-5-(tert-Butyldimethylsilanyloxymethyl)hexahydrofuro[3,2-b]furan-2-yl]-methanol (ent-4)

A solution of TBHP $(5.33 \mathrm{M}$ in isooctane, $1.3 \mathrm{~mL}, 6.7 \mathrm{mmol}, 1.0$ equiv) was added at room temperature to a solution of $\mathbf{1 8}(1.83 \mathrm{~g}, 6.7 \mathrm{mmol}, 1.0$ equiv) and $\mathrm{Co}^{\mathrm{II}}(\mathrm{Modp})^{2}$ (361 mg, $0.67 \mathrm{mmol}, 0.1$ equiv) in $i \mathrm{PrOH}(65 \mathrm{~mL})$. The reaction mixture was heated to $50{ }^{\circ} \mathrm{C}$ under $1 \mathrm{~atm}$ of $\mathrm{O}_{2}$ (balloon) for $16 \mathrm{~h}$, then concentrated under reduced pressure and purified by flash chromatography on silica gel (elution gradient: 33\% EtOAc/hexanes to 50\% EtOAc/hexanes) to give the title compound as a light green oil (1.58 g, 82\%). 
Rf 0.18 (50\% EtOAc/hexanes); ${ }^{1} \mathrm{H}$ NMR (600 MHz, $\left.\mathrm{C}_{6} \mathrm{D}_{6}\right) \delta 4.47(\mathrm{t}, J=4.4 \mathrm{~Hz}, 1 \mathrm{H}), 4.42(\mathrm{t}, J=$ $4.5 \mathrm{~Hz}, 1 \mathrm{H}), 4.11$ (ddt, $J=9.7,5.9,4.1 \mathrm{~Hz}, 1 \mathrm{H}), 4.05$ (ddt, $J=10.2,5.3,3.2 \mathrm{~Hz}, 1 \mathrm{H}), 3.59$ (dd, $J$ $=10.8,3.8 \mathrm{~Hz}, 1 \mathrm{H}), 3.56-3.52(\mathrm{~m}, 1 \mathrm{H}), 3.47(\mathrm{dd}, J=10.8,4.1 \mathrm{~Hz}, 1 \mathrm{H}), 3.27-3.23(\mathrm{~m}, 1 \mathrm{H}), 2.04$ (brs, $1 \mathrm{H}), 2.01(\mathrm{dd}, J=13.3,6.0 \mathrm{~Hz}, 1 \mathrm{H}), 1.85(\mathrm{dd}, J=13.2,5.6 \mathrm{~Hz}, 1 \mathrm{H}), 1.72$ (ddd, $J=13.2$, 9.1, $5.1 \mathrm{~Hz}, 1 \mathrm{H}), 1.56(\mathrm{ddd}, J=13.2,10.0,5.0 \mathrm{~Hz}, 1 \mathrm{H}), 0.97(\mathrm{~s}, 9 \mathrm{H}), 0.06(\mathrm{~s}, 6 \mathrm{H}) ;{ }^{13} \mathrm{C} \mathrm{NMR}$ $\left(150 \mathrm{MHz}, \mathrm{C}_{6} \mathrm{D}_{6}\right) \delta 84.7,84.5,80.7,80.6,65.7,64.1,37.1,36.5,26.1,18.5,-5.2,-5.2$; HRMS $m / z, 289.1840$ (calc'd for $\mathrm{C}_{14} \mathrm{H}_{29} \mathrm{O}_{4} \mathrm{Si}[\mathrm{M}+\mathrm{H}]^{+}, 289.1835$ ).

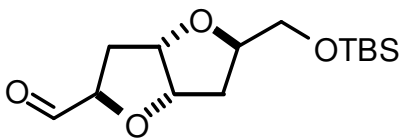

\section{(2R,3aS,5R,6aS)-5-(tert-Butyldimethylsilanyloxymethyl)- hexahydrofuro[3,2-b]furan-2-carbaldehyde (9)}

A solution of oxalyl chloride $(0.46 \mathrm{~mL}, 5.3 \mathrm{mmol}, 1.1$ equiv) in $\mathrm{CH}_{2} \mathrm{Cl}_{2}(17 \mathrm{~mL})$ was cooled to $-78{ }^{\circ} \mathrm{C}$ and DMSO (0.75 mL, $10.6 \mathrm{mmol}, 2.2$ equiv) in $\mathrm{CH}_{2} \mathrm{Cl}_{2}$ $(17 \mathrm{~mL})$ was added dropwise. After $1 \mathrm{~h}$ ent-4 (1.40 g, $4.8 \mathrm{mmol}, 1.0$ equiv) in $\mathrm{CH}_{2} \mathrm{Cl}_{2}(9.5 \mathrm{~mL})$ was added and after another $1.5 \mathrm{~h} \mathrm{Et}_{3} \mathrm{~N}(3.3 \mathrm{~mL}, 24 \mathrm{mmol}, 5.0$ equiv) was added. The solution was maintained at $-78^{\circ} \mathrm{C}$ for $0.5 \mathrm{~h}$, then allowed to warm to room temperature and stand for 0.5 h. The organic layer was washed with saturated aqueous $\mathrm{NaCl}(50 \mathrm{~mL})$ and the aqueous layer was back extracted with $\mathrm{CH}_{2} \mathrm{Cl}_{2}(2 \times 20 \mathrm{~mL})$. The organic extracts were combined, dried $\left(\mathrm{MgSO}_{4}\right)$, concentrated under reduced pressure and purified by flash chromatography on silica gel (elution gradient: $25 \% \mathrm{EtOAc/hexanes} \mathrm{to} 50 \% \mathrm{EtOAc/hexanes)} \mathrm{to} \mathrm{give} \mathrm{the} \mathrm{title} \mathrm{compound} \mathrm{as}$ a colorless oil $(1.20 \mathrm{~g}, 86 \%)$.

Rf 0.45 (50\% EtOAc/hexanes); ${ }^{1} \mathrm{H}$ NMR $\left(600 \mathrm{MHz}, \mathrm{C}_{6} \mathrm{D}_{6}\right) \delta 9.33(\mathrm{~d}, J=1.8 \mathrm{~Hz}, 1 \mathrm{H}), 4.32(\mathrm{t}, J$ $=4.7 \mathrm{~Hz}, 1 \mathrm{H}), 4.27(\mathrm{t}, J=4.4 \mathrm{~Hz}, 1 \mathrm{H}), 4.11(\mathrm{ddd}, J=8.8,6.4,1.8 \mathrm{~Hz}, 1 \mathrm{H}), 3.97$ (ddt, $J=9.4$, $5.9,3.5 \mathrm{~Hz}, 1 \mathrm{H}), 3.53(\mathrm{dd}, J=10.8,3.8 \mathrm{~Hz}, 1 \mathrm{H}), 3.38(\mathrm{dd}, J=11.1,4.1 \mathrm{~Hz}), 1.96-1.91(\mathrm{~m}, 2 \mathrm{H})$, 1.64 (ddd, $J=13.5,9.4,5.3 \mathrm{~Hz}, 1 \mathrm{H}), 1.43$ (ddd, $J=13.5,9.4,5.3 \mathrm{~Hz}, 1 \mathrm{H}), 0.95$ (s, 9H), 0.04 (s, $6 \mathrm{H}) ;{ }^{13} \mathrm{C}$ NMR $\left(150 \mathrm{MHz}, \mathrm{C}_{6} \mathrm{D}_{6}\right) \delta 200.1,85.9,83.6,83.4,80.5,65.3,36.3,35.8,26.0,18.5$, 5.2, -5.3; HRMS m/z 287.1670 (calc'd for $\mathrm{C}_{14} \mathrm{H}_{27} \mathrm{O}_{4} \mathrm{Si}[\mathrm{M}+\mathrm{H}]^{+}, 287.1679$ ).

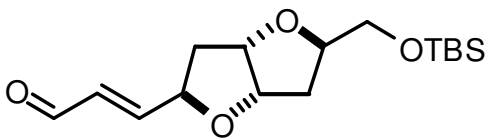

(E)-3-[(2R,3aS,5R,6aS)-5-(tertButyldimethylsilanyloxymethyl)-hexahydrofuro[3,2-b]furan2-yl]-propenal (11)

A solution of formylmethylenetriphenylphosphorane (1.25 g, $4.1 \mathrm{mmol}, 1.0$ equiv) and 9 (1.15 g, $4.0 \mathrm{mmol}, 1.0$ equiv) in MeCN (16 mL) was heated to reflux for $2 \mathrm{~h}$, then concentrated under reduced pressure and purified by flash chromatography on silica gel (elution gradient: $25 \%$ $\mathrm{EtOAc/hexanes} \mathrm{to} 33 \% \mathrm{EtOAc/hexanes)}$ to give the title compound as a light yellow oil (1.11 g, $88 \%)$.

$\mathrm{R} f 0.36$ (33\% EtOAc/hexanes); ${ }^{1} \mathrm{H}$ NMR $\left(600 \mathrm{MHz}, \mathrm{C}_{6} \mathrm{D}_{6}\right) \delta 9.31(\mathrm{~d}, J=7.6 \mathrm{~Hz}, 1 \mathrm{H}), 6.22$ (ddd, $J=15.7,7.8,1.8 \mathrm{~Hz}, 1 \mathrm{H}), 6.03(\mathrm{dd}, J=15.5,4.4 \mathrm{~Hz}, 1 \mathrm{H}), 4.41-4.34(\mathrm{~m}, 3 \mathrm{H}), 4.05$ (ddt, $J=$ 10.0, 6.4, 4.1 Hz, 1H), 3.56 (dd, $J=11.1,4.1 \mathrm{~Hz}, 1 \mathrm{H}), 3.42$ (dd, $J=10.5,4.1 \mathrm{~Hz}, 1 \mathrm{H}), 1.94$ (dd, $J=12.9,5.9 \mathrm{~Hz}, 2 \mathrm{H}), 1.69$ (ddd, $J=13.5,8.8,5.3 \mathrm{~Hz}, 1 \mathrm{H}), 1.14$ (ddd, $J=12.9,10.0,4.1 \mathrm{~Hz}$, $1 \mathrm{H}), 0.96(\mathrm{~s}, 9 \mathrm{H}), 0.06(\mathrm{~s}, 6 \mathrm{H}) ;{ }^{13} \mathrm{C}$ NMR $\left(150 \mathrm{MHz}, \mathrm{C}_{6} \mathrm{D}_{6}\right) \delta 192.2,154.9,130.8,84.8,84.1$, 80.9, 77.9, 41.0, 36.8, 26.1, 18.5, -5.2, -5.3; HRMS m/z 313.1842 (calc'd for $\mathrm{C}_{16} \mathrm{H}_{29} \mathrm{O}_{4} \mathrm{Si}$ $\left.[\mathrm{M}+\mathrm{H}]^{+}, 313.1835\right)$. 


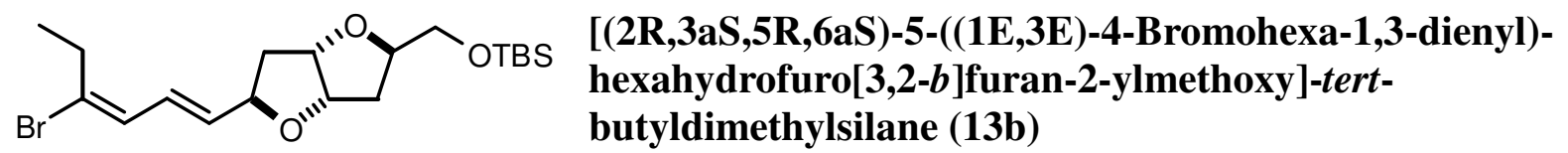

To a suspension of $12(1.54 \mathrm{~g}, 2.8 \mathrm{mmol}, 1.1$ equiv) in THF $(25 \mathrm{~mL})$ was added $n \mathrm{BuLi}(2.50 \mathrm{M}$ in hexanes, $1.0 \mathrm{~mL}, 2.5 \mathrm{mmol}, 1.0$ equiv) at $-78^{\circ} \mathrm{C}$. The mixture was maintained at $-78{ }^{\circ} \mathrm{C}$ for 1 $\mathrm{h}$ before a solution of $\mathbf{1 1}(780 \mathrm{mg}, 2.5 \mathrm{mmol}, 1.0$ equiv) in THF $(15 \mathrm{~mL})$ was added, and the mixture was maintained at $-78{ }^{\circ} \mathrm{C}$ for another $1.5 \mathrm{~h}$, allowed to warm to room temperature, concentrated under reduced pressure, filtered through a pad of silica gel and washed with $25 \%$ EtOAc/hexanes. The filtrate was concentrated under reduced pressure and purified by flash chromatography on silica gel (5\% $\mathrm{Et}_{2} \mathrm{O} /$ hexanes) to give $\mathbf{1 3 b}(368 \mathrm{mg}, 35 \%)$ as a colorless oil, 13a (377 mg, 36\%) as a colorless oil, and their mixture (107 mg, 11\%).

Rf $0.27\left(10 \%\right.$ EtOAc/hexanes); ${ }^{1} \mathrm{H}$ NMR $\left(600 \mathrm{MHz}, \mathrm{CDCl}_{3}\right) \delta 6.41(\mathrm{~d}, J=11.1 \mathrm{~Hz}, 1 \mathrm{H}), 6.33$ $(\mathrm{dd}, J=15.8,11.1 \mathrm{~Hz}, 1 \mathrm{H}), 5.62(\mathrm{dd}, J=14.9,6.7 \mathrm{~Hz}, 1 \mathrm{H}), 4.77(\mathrm{t}, J=4.4 \mathrm{~Hz}, 1 \mathrm{H}), 4.71(\mathrm{t}, J=$ $4.1 \mathrm{~Hz}, 1 \mathrm{H}), 4.54-4.50(\mathrm{~m}, 1 \mathrm{H}), 4.21-4.17(\mathrm{~m}, 1 \mathrm{H}), 3.67(\mathrm{dd}, J=11.1,4.1 \mathrm{~Hz}, 1 \mathrm{H}), 3.59(\mathrm{dd}, J$ $=11.1,4.1 \mathrm{~Hz}, 1 \mathrm{H}), 2.57(\mathrm{q}, J=7.6 \mathrm{~Hz}, 2 \mathrm{H}), 2.23(\mathrm{dd}, J=13.5,5.3 \mathrm{~Hz}, 1 \mathrm{H}), 2.10(\mathrm{dd}, J=13.5$, $5.9 \mathrm{~Hz}, 1 \mathrm{H}), 1.94$ (ddd, $J=13.8,8.5,5.3 \mathrm{~Hz}, 1 \mathrm{H}), 1.68(\mathrm{ddd}, J=13.0,10.0,4.7 \mathrm{~Hz}, 1 \mathrm{H}), 1.12(\mathrm{t}$, $J=7.3 \mathrm{~Hz}, 3 \mathrm{H}), 0.89(\mathrm{~s}, 9 \mathrm{H}), 0.06(\mathrm{~s}, 6 \mathrm{H}) ;{ }^{13} \mathrm{C} \mathrm{NMR}\left(150 \mathrm{MHz}, \mathrm{CDCl}_{3}\right) \delta 133.5,131.8,130.4$, $125.6,84.3,84.2,80.8,79.4,65.6,41.6,36.8,29.6,25.9,18.3,13.3,-5.3,-5.4$; HRMS m/z 416.1403 (calc'd for $\mathrm{C}_{19} \mathrm{H}_{33} \mathrm{BrO}_{3} \mathrm{Si}[\mathrm{M}]^{+}, 416.1382$ ).

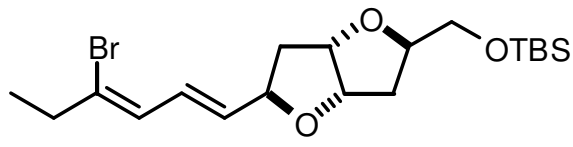

\section{[(2R,3aS,5R,6aS)-5-((1E,3Z)-4-Bromohexa-1,3-dienyl)- hexahydrofuro[3,2-b]furan-2-ylmethoxy]-tert- butyldimethylsilane (13a)}

$\mathrm{R} f 0.24\left(10 \%\right.$ EtOAc/hexanes); ${ }^{1} \mathrm{H}$ NMR $\left(600 \mathrm{MHz}, \mathrm{CDCl}_{3}\right) \delta 6.52(\mathrm{dd}, J=15.2,10.0,1 \mathrm{H}), 6.23$ $(\mathrm{d}, J=9.4 \mathrm{~Hz}, 1 \mathrm{H}), 5.72(\mathrm{dd}, J=15.2,7.0 \mathrm{~Hz}, 1 \mathrm{H}), 4.76(\mathrm{t}, J=4.4 \mathrm{~Hz}, 1 \mathrm{H}), 4.70(\mathrm{t}, J=4.4 \mathrm{~Hz}$, $1 \mathrm{H}$ ), 4.57-4.53 (m, 1H), 4.19 (dddd, $J=8.8,6.4,4.1,4.1 \mathrm{~Hz}, 1 \mathrm{H}), 3.67$ (dd, $J=11.1,4.1 \mathrm{~Hz}$, $1 \mathrm{H}), 3.58(\mathrm{dd}, J=11.1,4.1 \mathrm{~Hz}, 1 \mathrm{H}), 2.50(\mathrm{q}, J=7.6 \mathrm{~Hz}, 2 \mathrm{H}), 2.23(\mathrm{dd}, J=13.5,5.3 \mathrm{~Hz}, 1 \mathrm{H})$, $2.10(\mathrm{dd}, J=13.5,5.9 \mathrm{~Hz}, 1 \mathrm{H}), 1.93(\mathrm{ddd}, J=14.1,8.8,5.9 \mathrm{~Hz}, 1 \mathrm{H}), 1.68(\mathrm{ddd}, J=13.2,10.2$, $4.7 \mathrm{~Hz}, 1 \mathrm{H}), 1.12(\mathrm{t}, J=7.3 \mathrm{~Hz}, 3 \mathrm{H}), 0.88(\mathrm{~s}, 9 \mathrm{H}), 0.05(\mathrm{~s}, 6 \mathrm{H}) ;{ }^{13} \mathrm{C} \mathrm{NMR}\left(150 \mathrm{MHz}, \mathrm{CDCl}_{3}\right) \delta$ $134.5,131.9,129.3,125.7,84.4,84.2,80.8,79.6,65.6,41.6,36.8,35.3,25.9,18.3,13.4,-5.3$, 5.4; HRMS $m / z 416.1377$ (calc'd for $\mathrm{C}_{19} \mathrm{H}_{33} \mathrm{BrO}_{3} \mathrm{Si}[\mathrm{M}]^{+}$, 416.1382).

\section{Ozonolysis of 13a and 13b}

A mixture of 13a and 13b (60 mg, $0.14 \mathrm{mmol}, 1.0$ equiv) in $\mathrm{MeOH}(2 \mathrm{~mL})$ was cooled to $-78{ }^{\circ} \mathrm{C}$ and then $\mathrm{O}_{3}$ was bubbled through until a blue color persisted. The solution was then purged with Ar for $5 \mathrm{~min}$, then $\mathrm{NaBH}_{4}(150 \mathrm{mg}, 3.9 \mathrm{mmol}, 28.0$ equiv) was added and the solution was maintained at $-78{ }^{\circ} \mathrm{C}$ for $45 \mathrm{~min}$, then allowed to warm to and stand at room temperature for 45 min. The mixture was then concentrated under reduced pressure and purified by flash chromatography on silica gel (elution gradient: $33 \% \mathrm{EtOAc/hexanes} \mathrm{to} \mathrm{50 \%} \mathrm{EtOAc/hexanes)} \mathrm{to}$ give a colorless oil (22 mg, 53\%), the spectral data of which was identical to ent-4. 


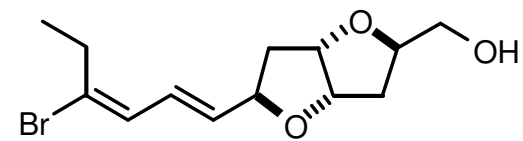

\section{[(2R,3aS,5R,6aS)-5-((1E,3E)-4-Bromohexa-1,3-dienyl)- hexahydrofuro[3,2-b]furan-2-yl]-methanol (19)}

To a solution of $\mathbf{1 3 b}(1.02 \mathrm{~g}, 2.4 \mathrm{mmol}, 1.0$ equiv) in THF (10 $\mathrm{mL}$ ) was added TBAF (1.0 M in THF, $3.6 \mathrm{~mL}, 3.6 \mathrm{mmol}, 1.5$ equiv) at room temperature. The mixture was allowed to stand at room temperature for $1 \mathrm{~h}$, then EtOAc $(10 \mathrm{~mL}), \mathrm{H}_{2} \mathrm{O}(5 \mathrm{~mL})$ and saturated aqueous $\mathrm{NaCl}(5 \mathrm{~mL})$ was added. The layers were separated, and the aqueous layer was extracted with EtOAc $(2 \times 20 \mathrm{~mL})$. The organic extracts were combined, dried $\left(\mathrm{MgSO}_{4}\right)$, concentrated under reduced pressure and purified by flash chromatography on silica gel (EtOAc) to give the title compound (700 mg, 96\%) as a yellow oil.

R $f 0.30$ (EtOAc); ${ }^{1} \mathrm{H}$ NMR $\left(600 \mathrm{MHz}, \mathrm{CDCl}_{3}\right) \delta 6.39(\mathrm{~d}, J=11.1 \mathrm{~Hz}, 1 \mathrm{H}), 6.31(\mathrm{dd}, J=14.6$, $11.1 \mathrm{~Hz}, 1 \mathrm{H}), 5.59(\mathrm{dd}, J=14.9,6.7 \mathrm{~Hz}, 1 \mathrm{H}), 4.75(\mathrm{t}, J=4.7 \mathrm{~Hz}, 1 \mathrm{H}), 4.71(\mathrm{t}, J=4.4 \mathrm{~Hz}, 1 \mathrm{H})$, $4.53-4.50(\mathrm{~m}, 1 \mathrm{H}), 4.23-4.19(\mathrm{~m}, 1 \mathrm{H}), 3.74(\mathrm{~d}, J=12.3 \mathrm{~Hz}, 1 \mathrm{H}), 3.48-3.44(\mathrm{~m}, 1 \mathrm{H}), 2.55(\mathrm{q}, J=$ $7.4 \mathrm{~Hz}, 2 \mathrm{H}), 2.24(\mathrm{dd}, J=13.2,5.0 \mathrm{~Hz}, 1 \mathrm{H}), 2.19(\mathrm{t}, J=5.3 \mathrm{~Hz}, 1 \mathrm{H}), 2.08(\mathrm{dd}, J=13.5,5.9 \mathrm{~Hz}$, $1 \mathrm{H}), 1.87$ (ddd, $J=13.5,10.0,4.7 \mathrm{~Hz}, 1 \mathrm{H}), 1.71$ (ddd, $J=13.5,10.3,4.4 \mathrm{~Hz}, 1 \mathrm{H}), 1.10$ (t, $J=$ $7.6 \mathrm{~Hz}, 3 \mathrm{H}) ;{ }^{13} \mathrm{C} \mathrm{NMR}\left(150 \mathrm{MHz}, \mathrm{CDCl}_{3}\right) \delta 133.3,132.0,130.3,125.7,84.2,84.1,80.6,79.9$, 64.1, 41.7, 36.2, 29.6, 13.3; HRMS $m / z, 302.0512$ (calc'd for $\mathrm{C}_{13} \mathrm{H}_{20} \mathrm{BrO}_{3}[\mathrm{M}+\mathrm{H}]^{+}, 302.0518$ ).

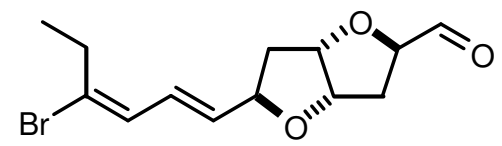

(2R,3aS,5R,6aS)-5-((1E,3E)-4-Bromohexa-1,3-dienyl)hexahydrofuro[3,2-b]furan-2-carbaldehyde (ent-3)

A solution of oxalyl chloride $(0.24 \mathrm{~mL}, 2.8 \mathrm{mmol}, 1.2$ equiv) in $\mathrm{CH}_{2} \mathrm{Cl}_{2}$ (7.2 mL) was cooled to $-78{ }^{\circ} \mathrm{C}$ and DMSO (0.39 mL, $5.5 \mathrm{mmol}, 2.4$ equiv) in $\mathrm{CH}_{2} \mathrm{Cl}_{2}$ (7.2 mL) was added dropwise. After $45 \mathrm{~min} 19$ (700 mg, $2.3 \mathrm{mmol}, 1.0$ equiv) in $\mathrm{CH}_{2} \mathrm{Cl}_{2}(4 \mathrm{~mL})$ was added and after another $1 \mathrm{~h} \mathrm{Et}_{3} \mathrm{~N}(1.6 \mathrm{~mL}, 11.5 \mathrm{mmol}, 5.0$ equiv) was added. The solution was maintained at $-78{ }^{\circ} \mathrm{C}$ for $15 \mathrm{~min}$, then allowed to warm to room temperature and stand for $15 \mathrm{~min}$. The organic layer was washed with saturated aqueous $\mathrm{NaCl}(20 \mathrm{~mL})$ and the aqueous layer was back extracted with $\mathrm{CH}_{2} \mathrm{Cl}_{2}(2 \times 20 \mathrm{~mL})$. The organic extracts were combined, dried $\left(\mathrm{MgSO}_{4}\right)$, concentrated under reduced pressure and purified by flash chromatography on silica gel (elution gradient: 50\% EtOAc/hexanes to $70 \%$ EtOAc/hexanes) to give the title compound as an orange oil (580 $\mathrm{mg}, 84 \%)$.

Rf 0.45 (75\% EtOAc/hexanes); ${ }^{1} \mathrm{H}$ NMR (600 MHz, $\left.\mathrm{CDCl}_{3}\right) \delta ;{ }^{13} \mathrm{C}$ NMR $\left(150 \mathrm{MHz}, \mathrm{CDCl}_{3}\right) \delta$ 200.6, 132.8, 132.3, 130.2, 126.1, 85.8, 83.7, 83.1, 79.8, 41.2, 35.9, 29.7, 13.3; HRMS m/z 300.0365 (calc'd for $\mathrm{C}_{13} \mathrm{H}_{17} \mathrm{BrO}_{3}[\mathrm{M}]^{+}, 300.0361$ ).

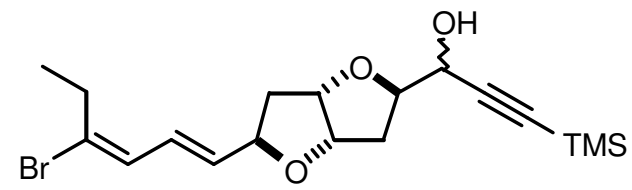

\section{1-[(2R,3aS,5R,6aS)-5-((1E,3E)-4-Bromohexa-1,3- dienyl)-hexahydrofuro[3,2- $b]$ furan-2-yl]-3- trimethylsilanylprop-2-yn-1-ol (ent-2)}

A solution of (trimethylsilyl)acetylene $(1.3 \mathrm{~mL}, 9.6 \mathrm{mmol}$, 5.0 equiv) in $\mathrm{Et}_{2} \mathrm{O}(40 \mathrm{~mL})$ was cooled to $0{ }^{\circ} \mathrm{C}$ and treated with $n \mathrm{BuLi}(2.60 \mathrm{M}$ in hexanes, 2.6 $\mathrm{mL}, 6.8 \mathrm{mmol}, 3.5$ equiv). After $10 \mathrm{~min}$ the solution was cooled to $-78{ }^{\circ} \mathrm{C}$ and $\mathrm{ClTi}(\mathrm{OiPr})_{3}(0.87$ $\mathrm{M}$ in hexanes, $7.8 \mathrm{~mL}, 6.8 \mathrm{mmol}, 3.5$ equiv) was added. After $30 \mathrm{~min}$ at $-78{ }^{\circ} \mathrm{C}$ ent-3 $(580 \mathrm{mg}$, $1.9 \mathrm{mmol}, 1.0$ equiv) in $\mathrm{Et}_{2} \mathrm{O}(10 \mathrm{~mL})$ was added. The solution was maintained at $-78{ }^{\circ} \mathrm{C}$ for an additional $1 \mathrm{~h}$, then slowly warmed to $-10{ }^{\circ} \mathrm{C}$ overnight. Aqueous L-tartaric acid ( $\left.1 \mathrm{M}, 63 \mathrm{~mL}\right)$ was added and the mixture was allowed to stand at room temperature for $1 \mathrm{~h}$, extracted with EtOAc $(3 \times 30 \mathrm{~mL})$, dried $\left(\mathrm{MgSO}_{4}\right)$, concentrated under reduced pressure and purified by flash 
chromatography on silica gel (elution gradient: $20 \%$ EtOAc/hexanes to $33 \%$ EtOAc/hexanes) to give the title compound as an orange oil $(623 \mathrm{mg}, 81 \%)$ consisting of two inseparable epimers.

Rf 0.31 (25\% EtOAc/hexanes); ${ }^{1} \mathrm{H}$ NMR $\left(600 \mathrm{MHz}, \mathrm{CDCl}_{3}\right) \delta 6.41(\mathrm{~d}, J=11.1 \mathrm{~Hz}, 1 \mathrm{H}), 6.34$ $(\mathrm{dd}, J=14.6,11.1 \mathrm{~Hz}, 1 \mathrm{H}), 5.62(\mathrm{dd}, J=14.9,6.7 \mathrm{~Hz}, 1 \mathrm{H}), 4.80-4.79(\mathrm{~m}, 2 \mathrm{H}), 4.78-4.74(\mathrm{~m}$, minor), 4.55-4.51 (m,1H), 4.47 (dd, $J=5.3,2.9 \mathrm{~Hz}, 1 \mathrm{H}), 4.31$ (ddd, $J=8.9,6.3,2.9 \mathrm{~Hz}, 1 \mathrm{H})$, 4.22-4.20 (m, minor), 2.57 (q, $J=7.4 \mathrm{~Hz}, 2 \mathrm{H}), 2.33$ (d, $J=5.3 \mathrm{~Hz}, 1 \mathrm{H}), 2.26$ (dd, $J=13.2,5.0$ $\mathrm{Hz}, 1 \mathrm{H}), 2.22-2.17(\mathrm{~m}, 2 \mathrm{H}), 1.72-1.67(\mathrm{~m}, 1 \mathrm{H}), 1.12(\mathrm{t}, J=7.3 \mathrm{~Hz}, 3 \mathrm{H}), 0.16(\mathrm{~s}, 9 \mathrm{H}) ;{ }^{13} \mathrm{C} \mathrm{NMR}$ $\left(150 \mathrm{MHz}, \mathrm{CDCl}_{3}\right) \delta 133.2,132.1,130.4,125.9,102.6,91.3,85.4,84.0,82.6,79.6,64.3,41.6$, 35.1, 29.7, 13.4, -0.2; HRMS m/z 398.0901 (calc'd for $\mathrm{C}_{18} \mathrm{H}_{27} \mathrm{BrO}_{3} \mathrm{Si}[\mathrm{M}]^{+}$, 398.0913).

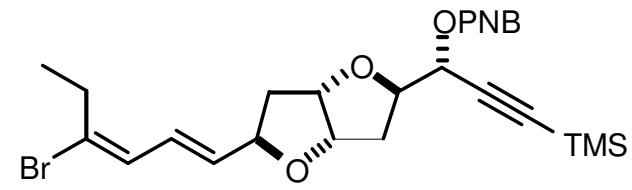

4-Nitrobenzoic acid (R)-1-[(2R,3aS,5R,6aS)-5((1E,3E)-4-bromohexa-1,3-dienyl)-hexahydrofuro[3,2b]furan-2-yl]-3-trimethylsilanylprop-2-ynyl ester (15b)

To a solution of ent-2 and $\mathbf{2 b}(593 \mathrm{mg}, 1.5 \mathrm{mmol}, 1.0$ equiv) in THF $(9 \mathrm{~mL})$ at $0{ }^{\circ} \mathrm{C}$ was added $\mathrm{Ph}_{3} \mathrm{P}(865 \mathrm{mg}, 3.3 \mathrm{mmol}, 2.2$ equiv) and $p$-nitrobenzoic acid (552 mg, $3.3 \mathrm{mmol}, 2.2$ equiv), then DIAD (0.65 mL, $3.3 \mathrm{mmol}, 2.2$ equiv) was added dropwise. The mixture was then allowed to stand at room temperature for $1 \mathrm{~h}$, filtered through a pad of silica and washed with $20 \%$ EtOAc/hexanes, then concentrated under reduced pressure and purified by flash chromatography on silica gel (elution gradient: $10 \% \mathrm{Et}_{2} \mathrm{O} /$ hexanes to $30 \%$ $\mathrm{Et}_{2} \mathrm{O} /$ hexanes) to give 15b as a light yellow oil (566 mg, 69\%) and 15a as a colorless oil (45 mg, $6 \%$ ), both of which turned into a white foam under vacuum.

Rf 0.47 (25\% EtOAc/hexanes); ${ }^{1} \mathrm{H}$ NMR $\left(600 \mathrm{MHz}, \mathrm{CDCl}_{3}\right) \delta 8.30(\mathrm{~d}, J=8.8 \mathrm{~Hz}, 2 \mathrm{H}), 8.25(\mathrm{~d}$, $J=8.8 \mathrm{~Hz}, 2 \mathrm{H}), 6.41(\mathrm{~d}, J=11.1 \mathrm{~Hz}, 1 \mathrm{H}), 6.34(\mathrm{dd}, J=14.6,11.1 \mathrm{~Hz}, 1 \mathrm{H}), 5.63-5.60(\mathrm{~m}, 2 \mathrm{H})$, $4.82(\mathrm{t}, J=4.4 \mathrm{~Hz}, 1 \mathrm{H}), 4.78(\mathrm{t}, J=4.1 \mathrm{~Hz}, 1 \mathrm{H}), 4.57-4.53(\mathrm{~m}, 1 \mathrm{H}), 4.47(\mathrm{q}, J=7.4 \mathrm{~Hz}, 1 \mathrm{H})$, $2.57(\mathrm{q}, J=7.2 \mathrm{~Hz}, 2 \mathrm{H}), 2.36(\mathrm{dd}, J=14.1,6.4 \mathrm{~Hz}, 1 \mathrm{H}), 2.26(\mathrm{dd}, J=13.5,4.7 \mathrm{~Hz}, 1 \mathrm{H}), 2.16$ (ddd, $J=13.9,8.3,5.3 \mathrm{~Hz}, 1 \mathrm{H}), 1.70(\mathrm{ddd}, J=13.5,10.5,4.7 \mathrm{~Hz}, 1 \mathrm{H}), 1.12(\mathrm{t}, J=7.3 \mathrm{~Hz}, 3 \mathrm{H})$, 0.17 (s, 9H); ${ }^{13} \mathrm{C}$ NMR $\left(150 \mathrm{MHz}, \mathrm{CDCl}_{3}\right) \delta 163.4,150.7,135.1,132.9,132.2,131.1,130.3$, 126.0, 123.5, 98.8, 93.1, 85.1, 83.4, 80.6, 79.5, 67.8, 41.3, 37.6, 29.7, 13.3, -0.4; HRMS $\mathrm{m} / z$ 547.1036 (calc'd for $\left.\mathrm{C}_{25} \mathrm{H}_{30} \mathrm{BrNO}_{6} \mathrm{Si}[\mathrm{M}]^{+}, 547.1026\right) ;[\alpha]_{\mathrm{D}}=-5.9^{\circ}\left(\mathrm{c}=0.025, \mathrm{CHCl}_{3}\right)$.

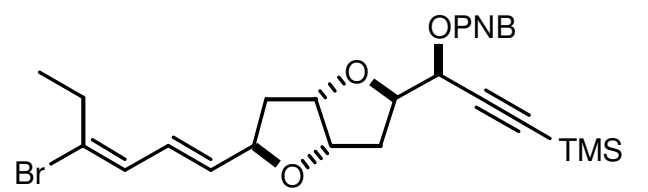

4-Nitrobenzoic acid (S)-1-[(2R,3aS,5R,6aS)-5-((1E,3E)4-bromohexa-1,3-dienyl)-hexahydrofuro[3,2-b]furan-

2-yl]-3-trimethylsilanylprop-2-ynyl ester (15a)

Rf 0.51 (25\% EtOAc/hexanes); ${ }^{1} \mathrm{H}$ NMR (600 MHz, $\left.\mathrm{CDCl}_{3}\right) \delta 8.29(\mathrm{~d}, J=9.4 \mathrm{~Hz}, 2 \mathrm{H}), 8.24(\mathrm{~d}, J=9.4 \mathrm{~Hz}, 2 \mathrm{H}), 6.41(\mathrm{~d}, J=11.1 \mathrm{~Hz}, 1 \mathrm{H}), 6.33(\mathrm{dd}, J$ $=15.2,11.1 \mathrm{~Hz}, 1 \mathrm{H}), 5.78(\mathrm{~d}, J=2.9 \mathrm{~Hz}, 1 \mathrm{H}), 5.61(\mathrm{dd}, J=14.9,6.7 \mathrm{~Hz}, 1 \mathrm{H}), 4.83(\mathrm{t}, J=4.4$ $\mathrm{Hz}, 1 \mathrm{H}), 4.78(\mathrm{t}, J=4.1 \mathrm{~Hz}, 1 \mathrm{H}), 4.55-4.49(\mathrm{~m}, 2 \mathrm{H}), 2.37(\mathrm{dd}, J=13.5,6.4 \mathrm{~Hz}, 1 \mathrm{H}), 2.31-2.23$ $(\mathrm{m}, 2 \mathrm{H}), 1.69(\mathrm{ddd}, J=13.5,10.3,4.4 \mathrm{~Hz}, 1 \mathrm{H}), 1.11(\mathrm{t}, J=7.3 \mathrm{~Hz}, 3 \mathrm{H}), 0.17(\mathrm{~s}, 9 \mathrm{H}) ;{ }^{13} \mathrm{C} \mathrm{NMR}$ $\left(150 \mathrm{MHz}, \mathrm{CDCl}_{3}\right) \delta 163.4,150.7,135.0,133.0,132.2,131.0,130.3,125.9,123.6,98.2,93.2$, 85.8, 83.5, 80.9, 79.7, 67.1, 41.4, 36.2, 29.7, 13.3, -0.4; HRMS $\mathrm{m} / \mathrm{z} 547.1020$ (calc'd for $\mathrm{C}_{25} \mathrm{H}_{30} \mathrm{BrNO}_{6} \mathrm{Si}[\mathrm{M}]^{+}$, 547.1026). 


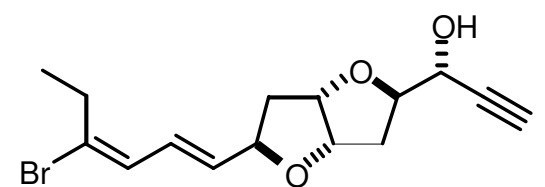

(R)-1-[(2R,3aS,5R,6aS)-5-((1E,3E)-4-Bromohexa-1,3dienyl)-hexahydrofuro[3,2-b]furan-2-yl]-prop-2-yn-1-ol

(16b)

A solution of $\mathbf{1 5 b}(140 \mathrm{mg}, 0.26 \mathrm{mmol}, 1.0$ equiv) in $\mathrm{MeOH}$ $(4.5 \mathrm{~mL})$ was stirred over $\mathrm{K}_{2} \mathrm{CO}_{3}\left(177 \mathrm{mg}, 1.28 \mathrm{mmol}, 5.0\right.$ equiv) at $0{ }^{\circ} \mathrm{C}$ for $1.5 \mathrm{~h}$. The mixture was then allowed to warm to room temperature, diluted with $\mathrm{Et}_{2} \mathrm{O}(10 \mathrm{~mL})$, filtered through Celite, concentrated under reduced pressure and purified by flash chromatography over silica gel (elution gradient: $20 \% \mathrm{EtOAc/hexanes} \mathrm{to} 50 \% \mathrm{EtOAc} / \mathrm{hexanes}$ ) to give the title compound as a colorless oil (77 mg, 92\%).

R $f 0.31\left(40 \%\right.$ EtOAc/hexanes); ${ }^{1} \mathrm{H}$ NMR $\left(600 \mathrm{MHz}, \mathrm{CDCl}_{3}\right) \delta 6.41(\mathrm{~d}, J=11.1 \mathrm{~Hz}, 1 \mathrm{H}), 6.33$ (dd, $J=14.6,11.1 \mathrm{~Hz}, 1 \mathrm{H}), 5.61(\mathrm{dd}, J=14.6,7.0 \mathrm{~Hz}, 1 \mathrm{H}), 4.78-4.75(\mathrm{~m}, 2 \mathrm{H}), 4.56-4.52(\mathrm{~m}$, $1 \mathrm{H}), 4.26-4.22(\mathrm{~m}, 2 \mathrm{H}), 2.57(\mathrm{q}, J=7.6 \mathrm{~Hz}, 2 \mathrm{H}), 2.48(\mathrm{~d}, J=1.8 \mathrm{~Hz}, 1 \mathrm{H}), 2.46(\mathrm{~d}, J=5.3 \mathrm{~Hz}$, $1 \mathrm{H}), 2.31(\mathrm{dd}, J=13.5,5.3 \mathrm{~Hz}, 1 \mathrm{H}), 2.24(\mathrm{dd}, J=13.5,5.3 \mathrm{~Hz}, 1 \mathrm{H}), 2.00(\mathrm{ddd}, J=13.6,8.6,5.3$ $\mathrm{Hz}, 1 \mathrm{H}), 1.71$ (ddd, $J=13.8,10.3,4.1 \mathrm{~Hz}, 1 \mathrm{H}), 1.12(\mathrm{t}, J=7.3 \mathrm{~Hz}, 3 \mathrm{H}) ;{ }^{13} \mathrm{C}$ NMR $(150 \mathrm{MHz}$, $\left.\mathrm{CDCl}_{3}\right) \delta 133.1,132.1,130.3,125.9,85.0,83.8,82.8,81.9,79.8,74.0,64.6,41.5,36.8,29.7$, 13.3; HRMS $m / z 326.0512$ (calc'd for $\mathrm{C}_{15} \mathrm{H}_{19} \mathrm{BrO}_{3}[\mathrm{M}]^{+}, 326.0518$ ).

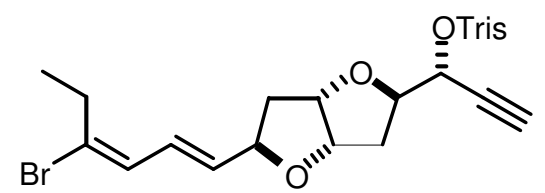

2,4,6-Triisopropyl-benzenesulfonic acid (R)-1[(2R,3aS,5R,6aS)-5-((1E,3E)-4-bromohexa-1,3-dienyl)hexahydrofuro[3,2-b]furan-2-yl]-prop-2-ynyl ester (14b) A solution of $\mathbf{1 6 b}$ ( $77 \mathrm{mg}, 0.24 \mathrm{mmol}, 1.0$ equiv), DMAP (87 $\mathrm{mg}, 0.72 \mathrm{mmol}, 3.0$ equiv) and 2,4,6-triisopropylbenzenesulfonyl chloride (148 mg, $0.48 \mathrm{mmol}$, 2.0 equiv) in $\mathrm{CH}_{2} \mathrm{Cl}_{2}(4.8 \mathrm{~mL})$ was allowed to stand at room temperature for $1.5 \mathrm{~h}$, filtered through a pad of silica and washed with $25 \%$ EtOAc/hexanes, then concentrated under reduced pressure and purified by flash chromatography over silica gel (10\% EtOAc/hexanes) to give the title compound as a colorless oil $(111 \mathrm{mg}, 78 \%)$.

Rf 0.36 (17\% EtOAc/hexanes); ${ }^{1} \mathrm{H}$ NMR (600 MHz, $\left.\mathrm{CDCl}_{3}\right) \delta 7.16$ (s, 2H), 6.40 (d, $J=11.1 \mathrm{~Hz}$, $1 \mathrm{H}), 6.32(\mathrm{dd}, J=15.2,11.1 \mathrm{~Hz}, 1 \mathrm{H}), 5.60(\mathrm{dd}, J=14.9,6.7 \mathrm{~Hz}, 1 \mathrm{H}), 5.15(\mathrm{dd}, J=5.3,1.8 \mathrm{~Hz}$, $1 \mathrm{H}), 4.78(\mathrm{t}, J=4.7 \mathrm{~Hz}, 1 \mathrm{H}), 4.73(\mathrm{t}, J=4.1 \mathrm{~Hz}, 1 \mathrm{H}), 4.50-4.47(\mathrm{~m}, 1 \mathrm{H}), 4.42-4.38(\mathrm{~m}, 1 \mathrm{H})$, 4.13 (septet, $J=7.0 \mathrm{~Hz}, 2 \mathrm{H}), 2.90$ (septet, $J=7.0 \mathrm{~Hz}, 1 \mathrm{H}), 2.57(\mathrm{q}, J=7.4 \mathrm{~Hz}, 2 \mathrm{H}), 2.30$ (d, $J=$ $2.3 \mathrm{~Hz}, 1 \mathrm{H}), 2.28(\mathrm{dd}, J=13.8,6.7 \mathrm{~Hz}, 1 \mathrm{H}), 2.24(\mathrm{dd}, J=13.5,5.3 \mathrm{~Hz}, 1 \mathrm{H}), 2.15(\mathrm{ddd}, J=13.9$, $8.3,5.3 \mathrm{~Hz}, 1 \mathrm{H}), 1.65(\mathrm{ddd}, J=13.5,10.5,4.1 \mathrm{~Hz}, 1 \mathrm{H}), 1.27(\mathrm{t}, J=6.7 \mathrm{~Hz}, 12 \mathrm{H}), 1.24(\mathrm{~d}, J=7.0$ $\mathrm{Hz}, 6 \mathrm{H}), 1.12(\mathrm{t}, J=7.3 \mathrm{~Hz}, 3 \mathrm{H}) ;{ }^{13} \mathrm{C}$ NMR $\left(150 \mathrm{MHz}, \mathrm{CDCl}_{3}\right) \delta 153.9,150.7,133.0,132.1$, 130.4, 130.3, 125.9, 123.6, 110.0, 85.3, 83.5, 80.4, 79.4, 76.7, 70.9, 41.2, 36.7, 34.2, 29.7, 24.7, 24.6, 23.6, 13.3; HRMS m/z 592.1855 (calc'd for $\mathrm{C}_{30} \mathrm{H}_{41} \mathrm{BrO}_{5} \mathrm{~S}[\mathrm{M}]^{+}$, 592.1858).

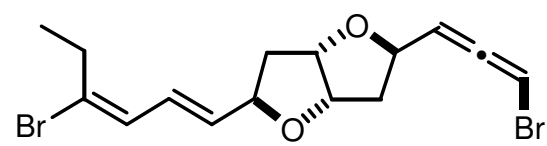

\section{Aplysiallene (1b)}

To a solution of 14b (111 $\mathrm{mg}, 0.18 \mathrm{mmol}, 1.0$ equiv) in THF $\mathrm{Br}(4 \mathrm{~mL})$ is added $\mathrm{LiCuBr}_{2}(0.25 \mathrm{M}$ in THF, $3.8 \mathrm{~mL}, 0.95 \mathrm{mmol}$, 5.0 equiv) and the mixture is heated to $70{ }^{\circ} \mathrm{C}$ for $2 \mathrm{~h}$, then cooled to room temperature and saturated aqueous $\mathrm{NH}_{4} \mathrm{Cl}(10 \mathrm{~mL})$ was added. The layers were separated and the aqueous layer was extracted with EtOAc $(3 \times 10 \mathrm{~mL})$. The organic extracts were combined, dried $\left(\mathrm{MgSO}_{4}\right)$, concentrated under reduced pressure and purified by flash chromatography over silica gel (10\% $\mathrm{Et}_{2} \mathrm{O} /$ hexanes) to give the title compound as a light yellow oil (42 mg, $\left.60 \%\right)$. 
$\mathrm{R} f 0.39$ (17\% EtOAc/hexanes); ${ }^{1} \mathrm{H}$ NMR $\left(600 \mathrm{MHz}, \mathrm{CDCl}_{3}\right) \delta 6.41(\mathrm{~d}, J=11.1 \mathrm{~Hz}, 1 \mathrm{H}), 6.33$ (ddd, $J=14.6,11.1,1.2 \mathrm{~Hz}, 1 \mathrm{H}), 6.09(\mathrm{dd}, J=5.9,1.8 \mathrm{~Hz}, 1 \mathrm{H}), 5.61(\mathrm{dd}, J=14.9,6.7 \mathrm{~Hz}, 1 \mathrm{H})$, $5.38(\mathrm{t}, J=6.2 \mathrm{~Hz}, 1 \mathrm{H}), 4.81(\mathrm{t}, J=5.0 \mathrm{~Hz}, 1 \mathrm{H}), 4.75-4.70(\mathrm{~m}, 2 \mathrm{H}), 4.54-4.50(\mathrm{~m}, 1 \mathrm{H}), 2.57(\mathrm{q}$, $J=7.2 \mathrm{~Hz}, 2 \mathrm{H}), 2.29(\mathrm{dd}, J=13.5,5.9 \mathrm{~Hz}, 1 \mathrm{H}), 2.26(\mathrm{dd}, J=13.8,5.0 \mathrm{~Hz}, 1 \mathrm{H}), 1.96(\mathrm{ddd}, J=$ $13.5,8.2,5.3 \mathrm{~Hz}, 1 \mathrm{H}), 1.73(\mathrm{ddd}, J=13.5,10.3,5.0 \mathrm{~Hz}, 1 \mathrm{H}), 1.13(\mathrm{t}, J=7.3 \mathrm{~Hz}, 3 \mathrm{H}) ;{ }^{13} \mathrm{C} \mathrm{NMR}$ $\left(150 \mathrm{MHz}, \mathrm{CDCl}_{3}\right) \delta 201.8,133.1,132.1,130.3,125.9,101.0,84.0,83.7,79.7,76.6,73.8,41.3$, 40.6, 29.7, 13.3; HRMS $m / z 389.9657$ (calc'd for $\mathrm{C}_{15} \mathrm{H}_{18}{ }^{79} \mathrm{Br}^{81} \mathrm{BrO}_{2}[\mathrm{M}]^{+}, 389.9653$ ); $[\alpha]_{\mathrm{D}}=-$ $192^{\circ}\left(\mathrm{c}=0.002, \mathrm{CHCl}_{3}\right)$.

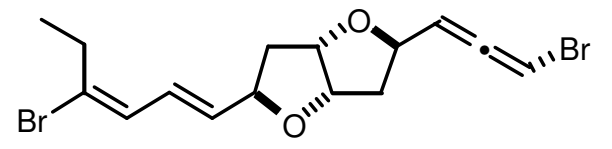

\section{1-epi-Aplysiallene (ent-1)}

$\mathrm{R} f 0.36$ (17\% EtOAc/hexanes); ${ }^{1} \mathrm{H}$ NMR $(600 \mathrm{MHz}$, $\left.\mathrm{CDCl}_{3}\right) \delta 6.41(\mathrm{~d}, J=11.1 \mathrm{~Hz}, 1 \mathrm{H}), 6.33(\mathrm{dd}, J=14.6,11.1 \mathrm{~Hz}, 1 \mathrm{H}), 6.07(\mathrm{dd}, J=5.6,1.5 \mathrm{~Hz}$, $1 \mathrm{H}), 5.61(\mathrm{dd}, J=15.2,7.0 \mathrm{~Hz}, 1 \mathrm{H}), 5.40(\mathrm{t}, J=5.6 \mathrm{~Hz}, 1 \mathrm{H}), 4.79(\mathrm{t}, J=4.4 \mathrm{~Hz}, 1 \mathrm{H}), 4.75-4.70$ (m, 2H), 4.54-4.50 (m, 1H), $2.57(\mathrm{q}, J=7.4 \mathrm{~Hz}, 2 \mathrm{H}), 2.30-2.25(\mathrm{~m}, 2 \mathrm{H}), 1.95$ (ddd, $J=13.6$, 8.3, 5.6 Hz, 1H), $1.73(\mathrm{ddd}, J=14.6,10.0,4.7 \mathrm{~Hz}, 1 \mathrm{H}), 1.12(\mathrm{t}, J=7.3 \mathrm{~Hz}, 3 \mathrm{H}),{ }^{13} \mathrm{C}$ NMR $(150$ $\left.\mathrm{MHz}, \mathrm{CDCl}_{3}\right) \delta 201.5,133.1,132.1,130.3,126.0,101.4,84.2,83.6,79.6,76.2,73.9,41.3,40.8$, 29.7, 13.3; HRMS $m / z 387.9706$ (calc'd for $\left.\mathrm{C}_{15} \mathrm{H}_{18}{ }^{79} \mathrm{Br}_{2} \mathrm{O}_{2}[\mathrm{M}]^{+}, 387.9674\right) ;[\alpha]_{\mathrm{D}}=+166^{\circ}(\mathrm{c}=$ $\left.0.002, \mathrm{CHCl}_{3}\right)$.

1. Gao, Y.; Klunder, J. M.; Hanson, R. M.; Masamune, H.; Ko, S. Y.; Sharpless, K. B. J. Am. Che. Soc. 1987, 109, 5765-5780.

2. Still, W. C.; Kahn, M.; Mitra, A. A. J. Org. Chem. 1978, 43, 2923-2925. 


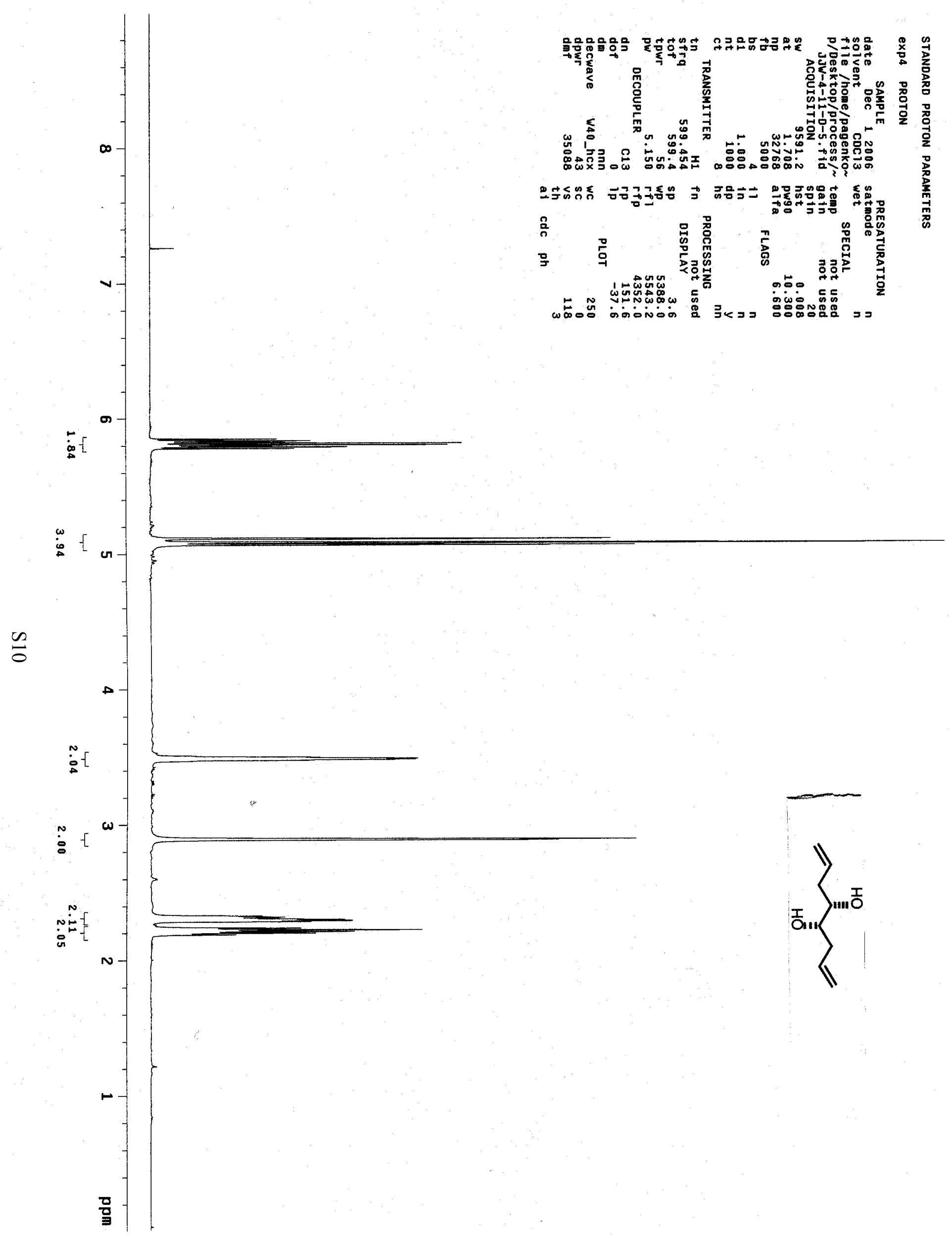




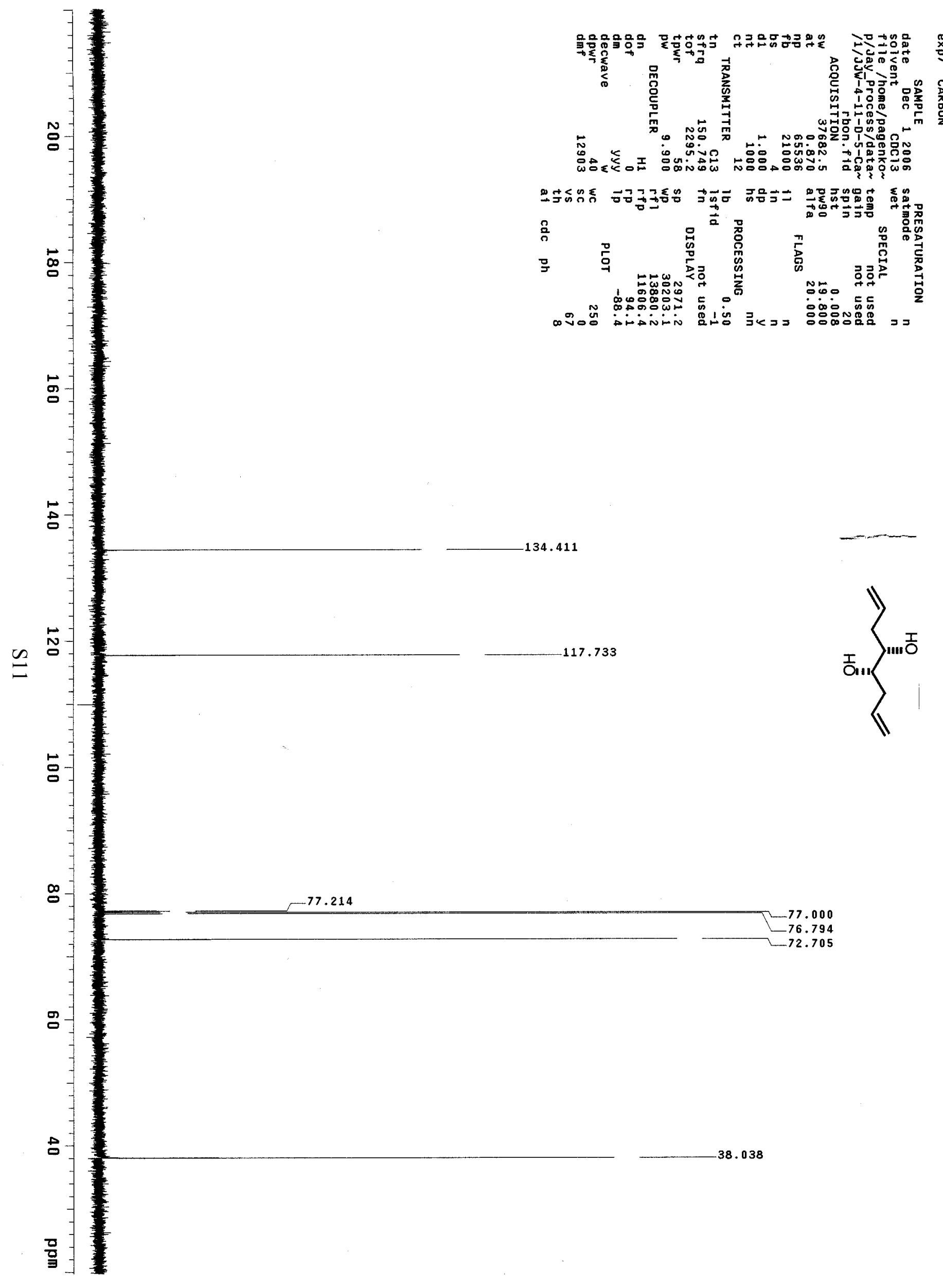




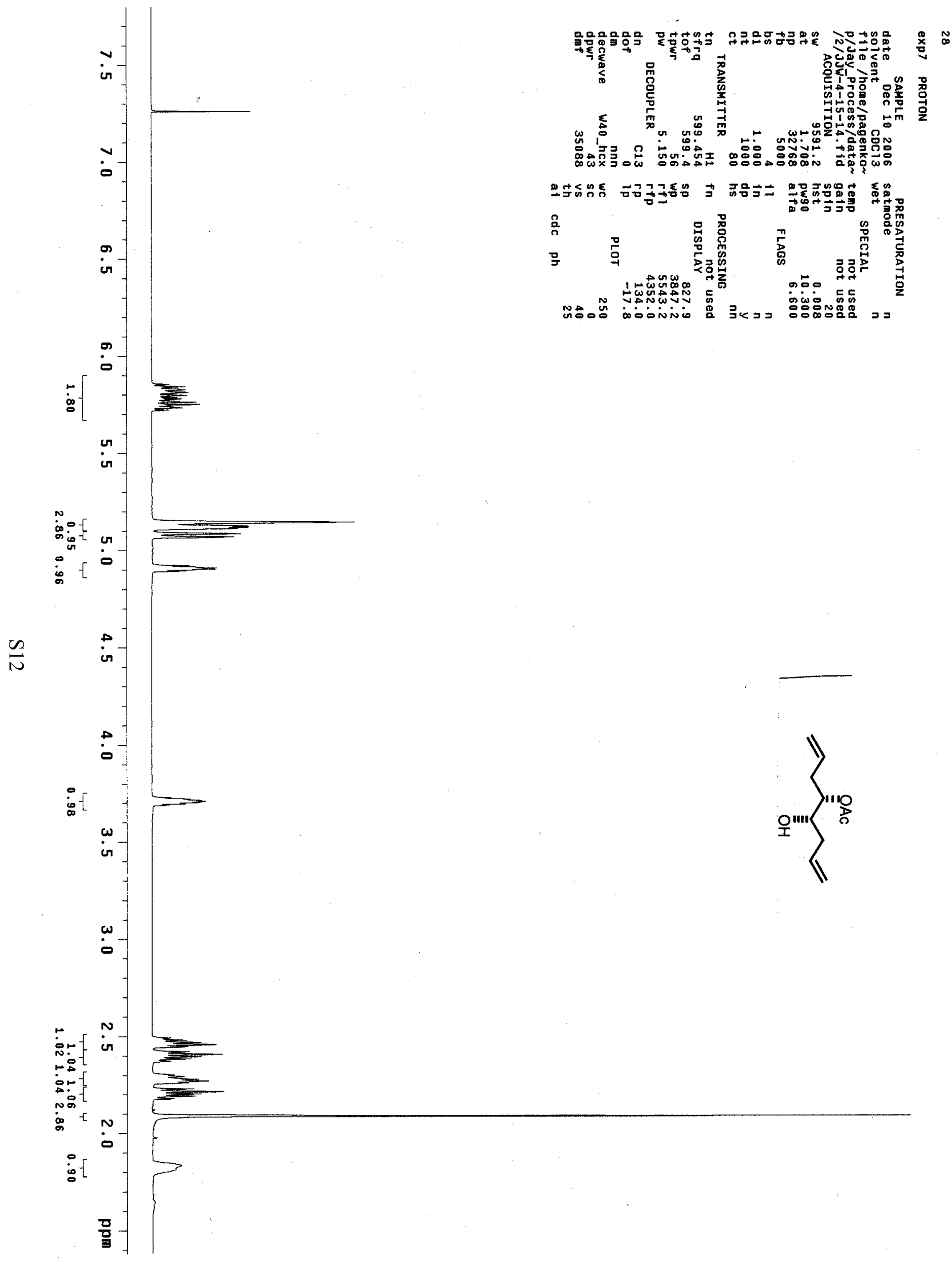




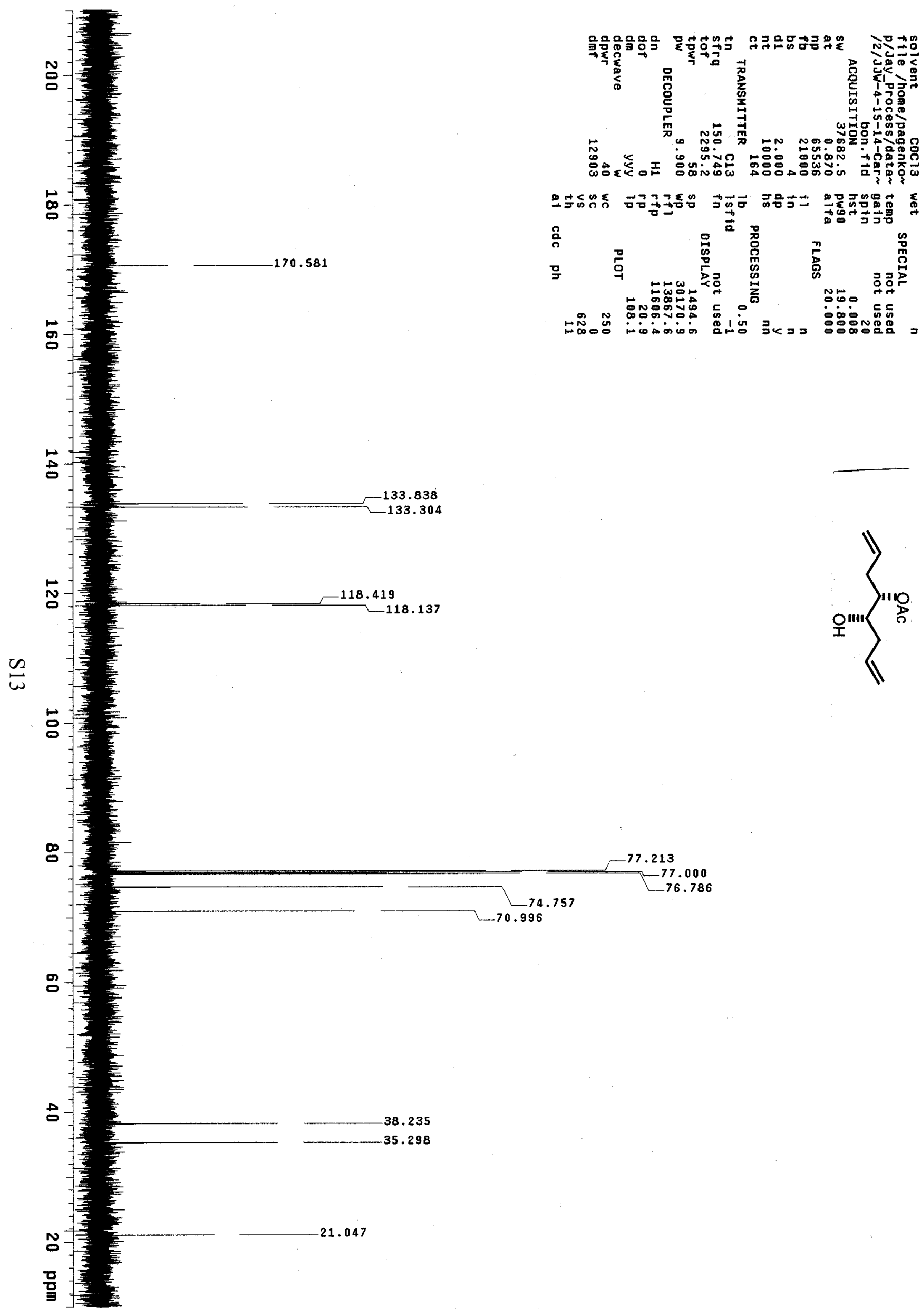




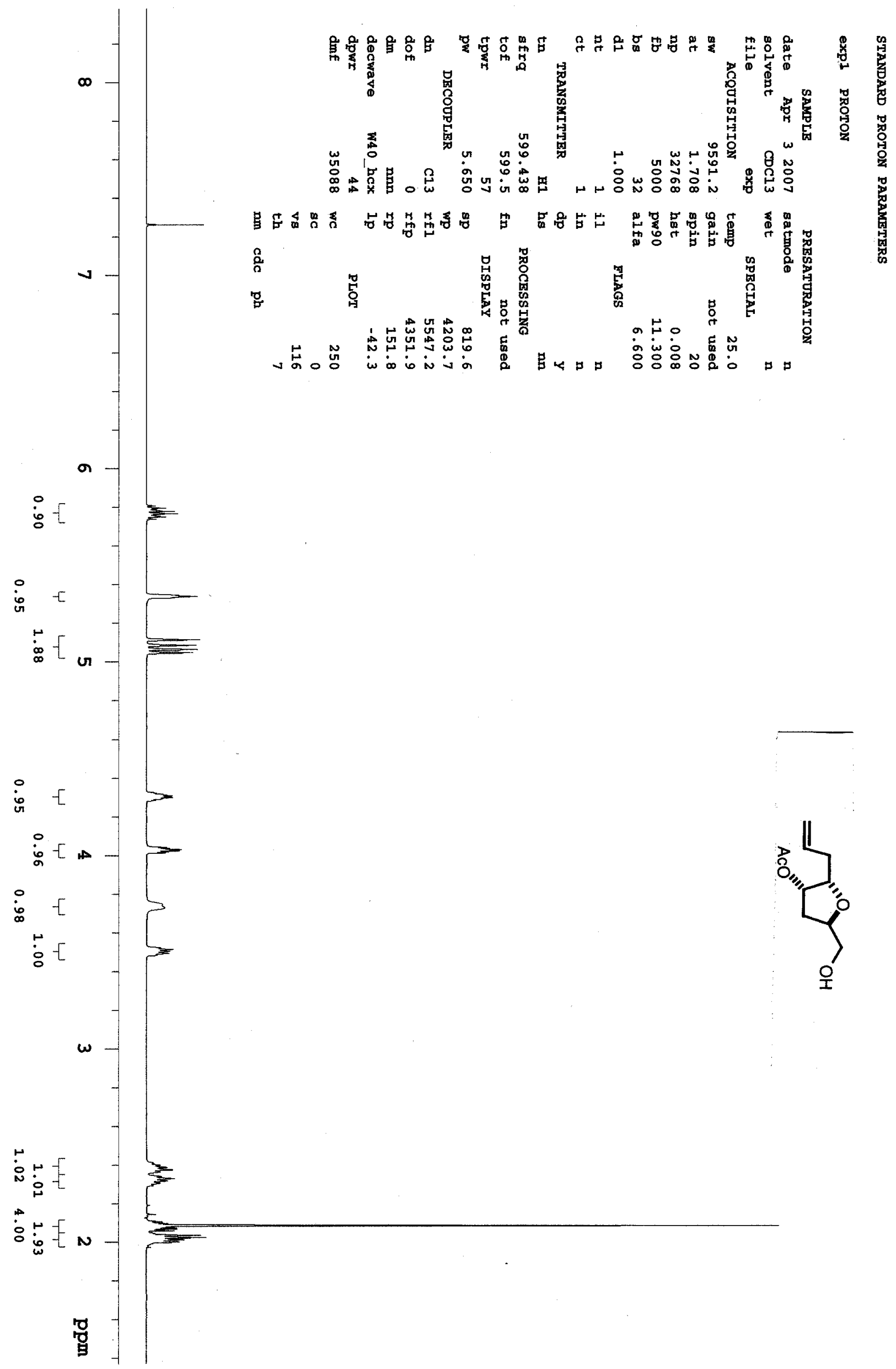




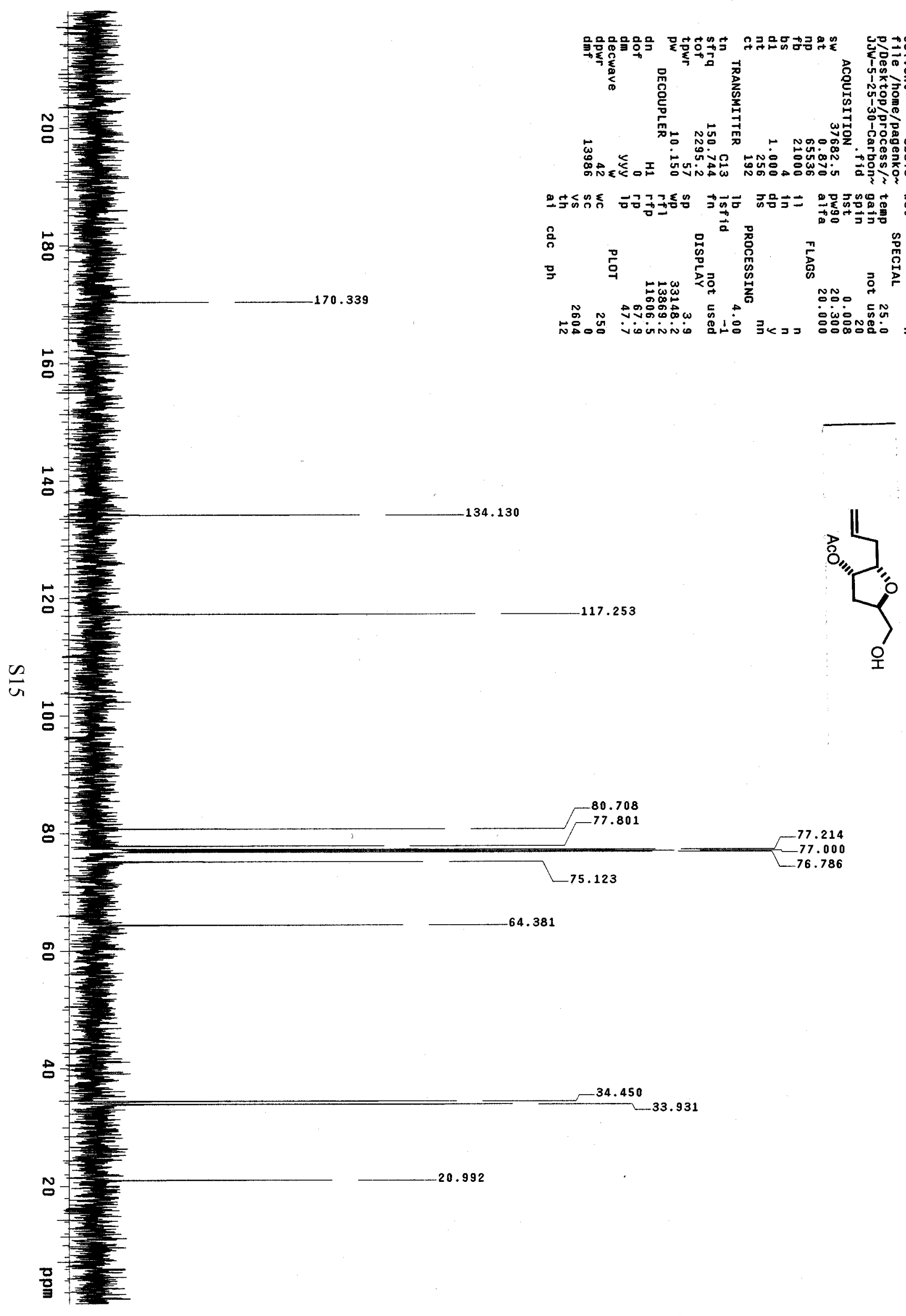




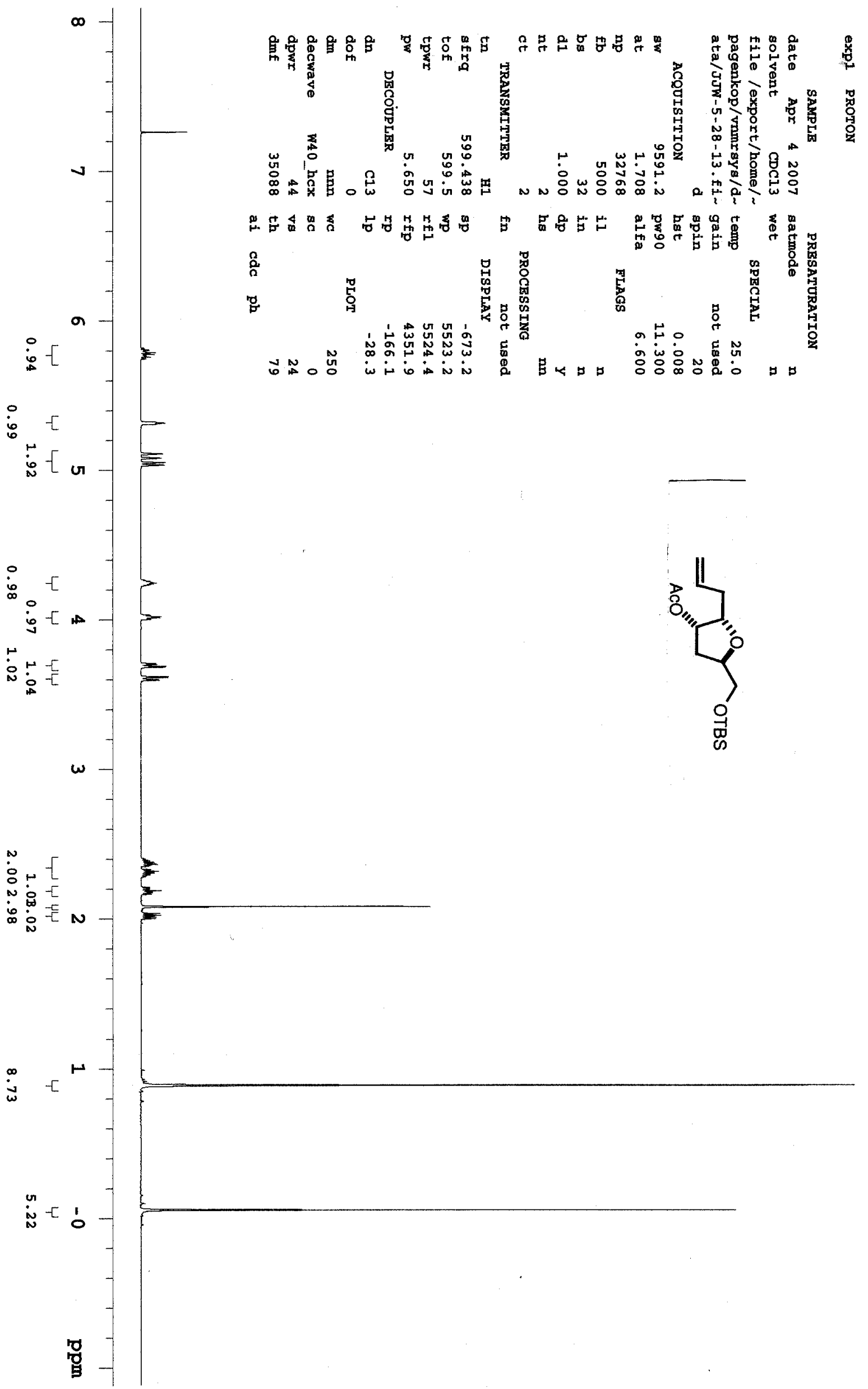




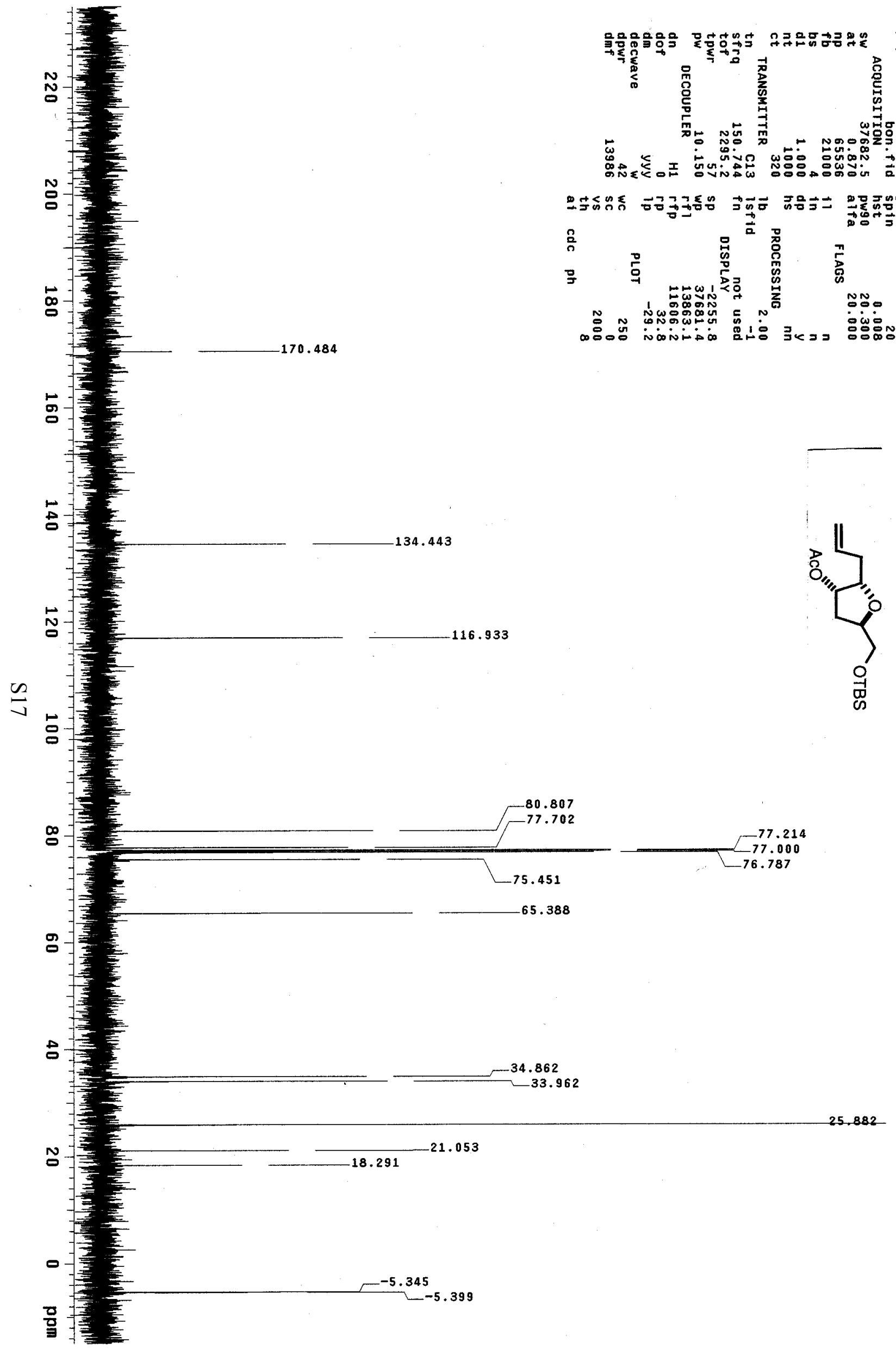




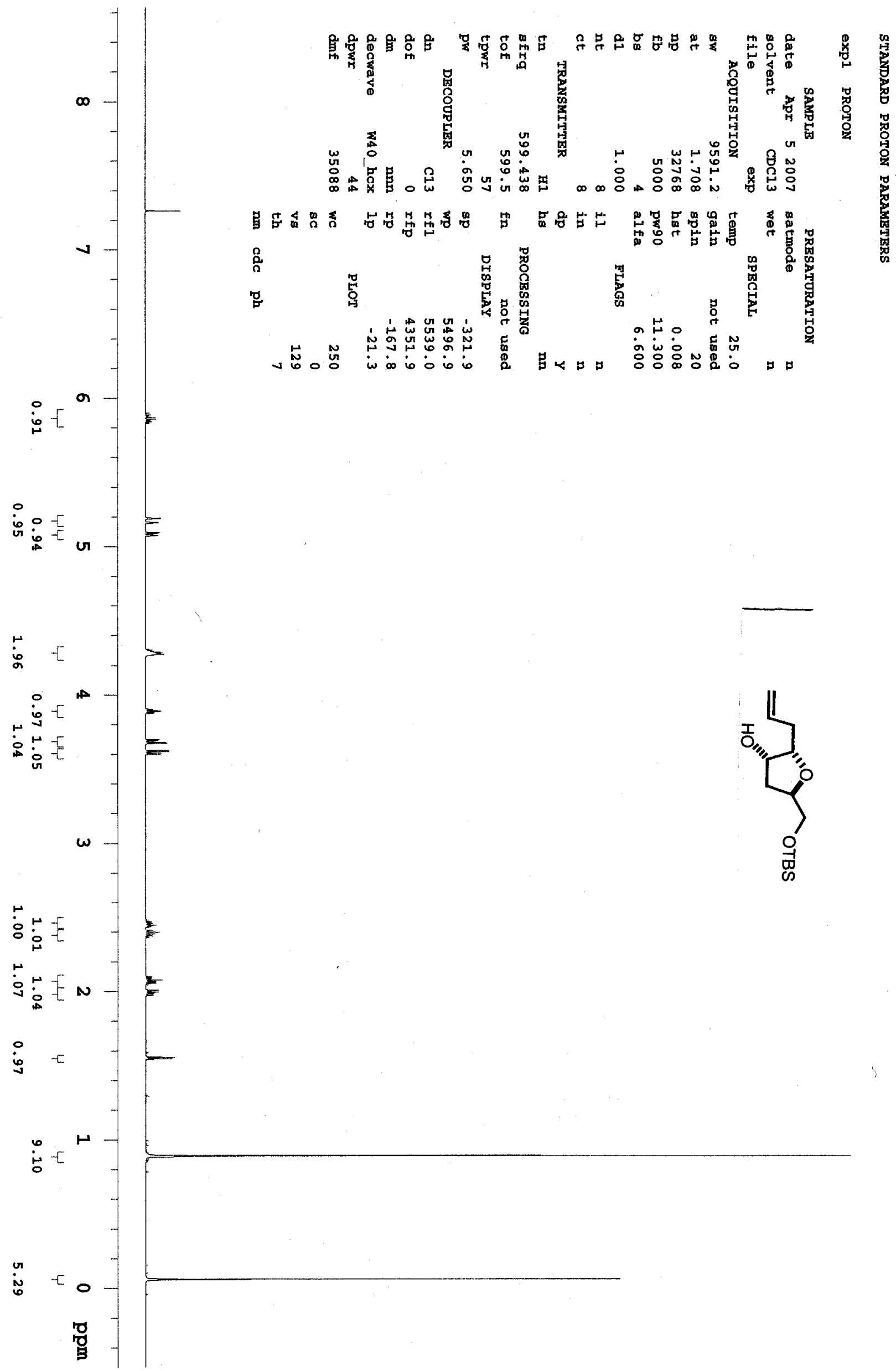




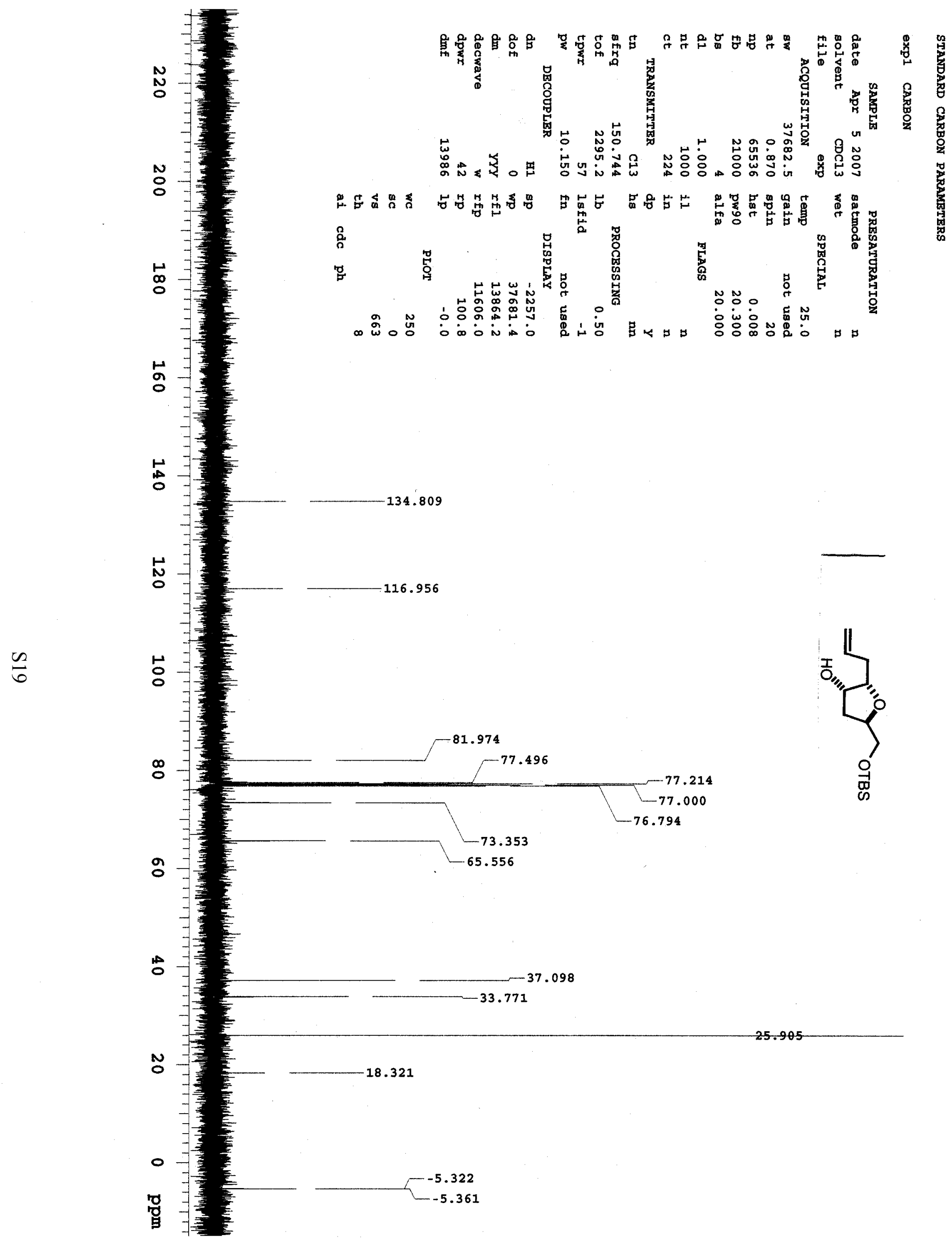




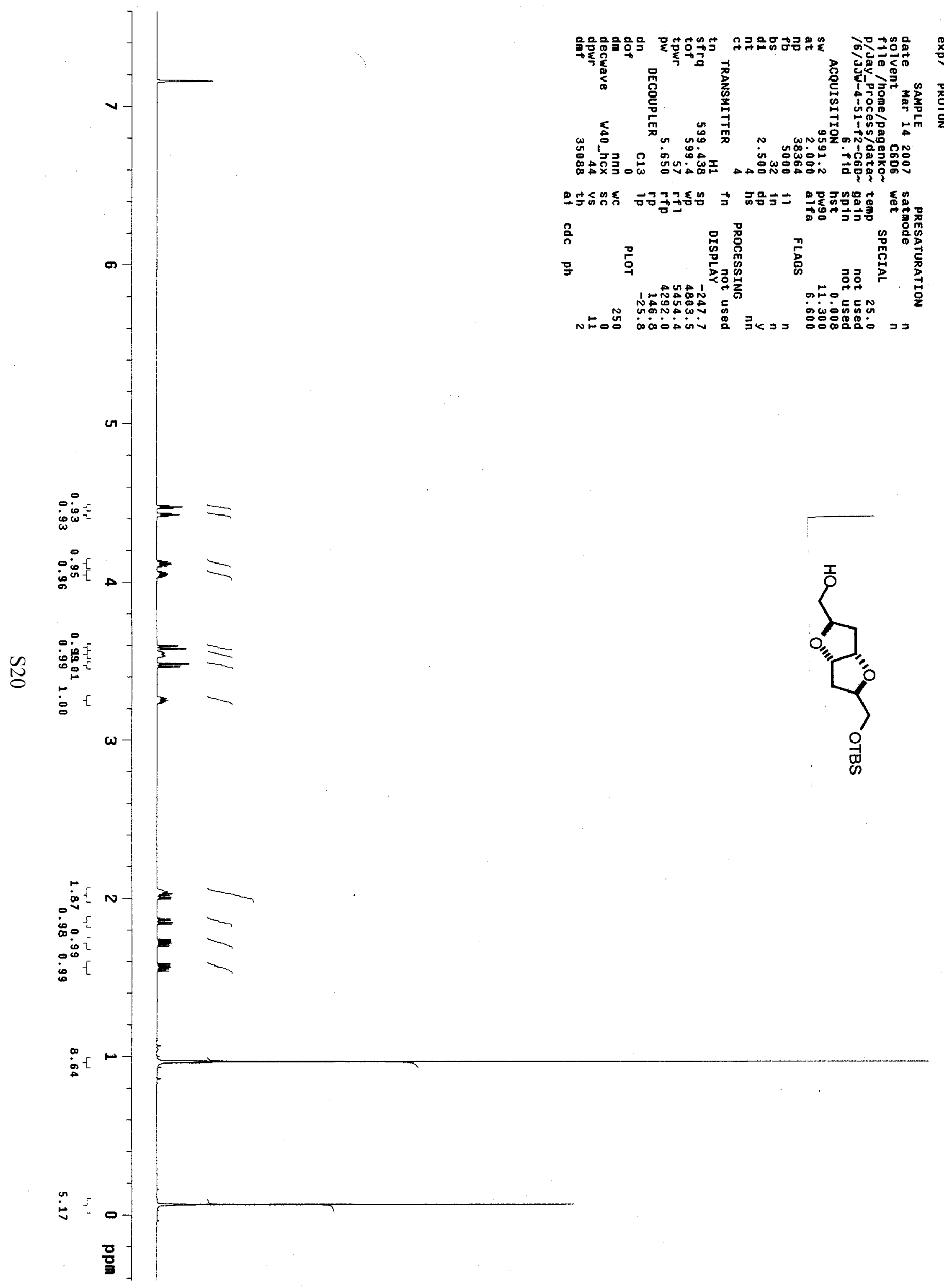




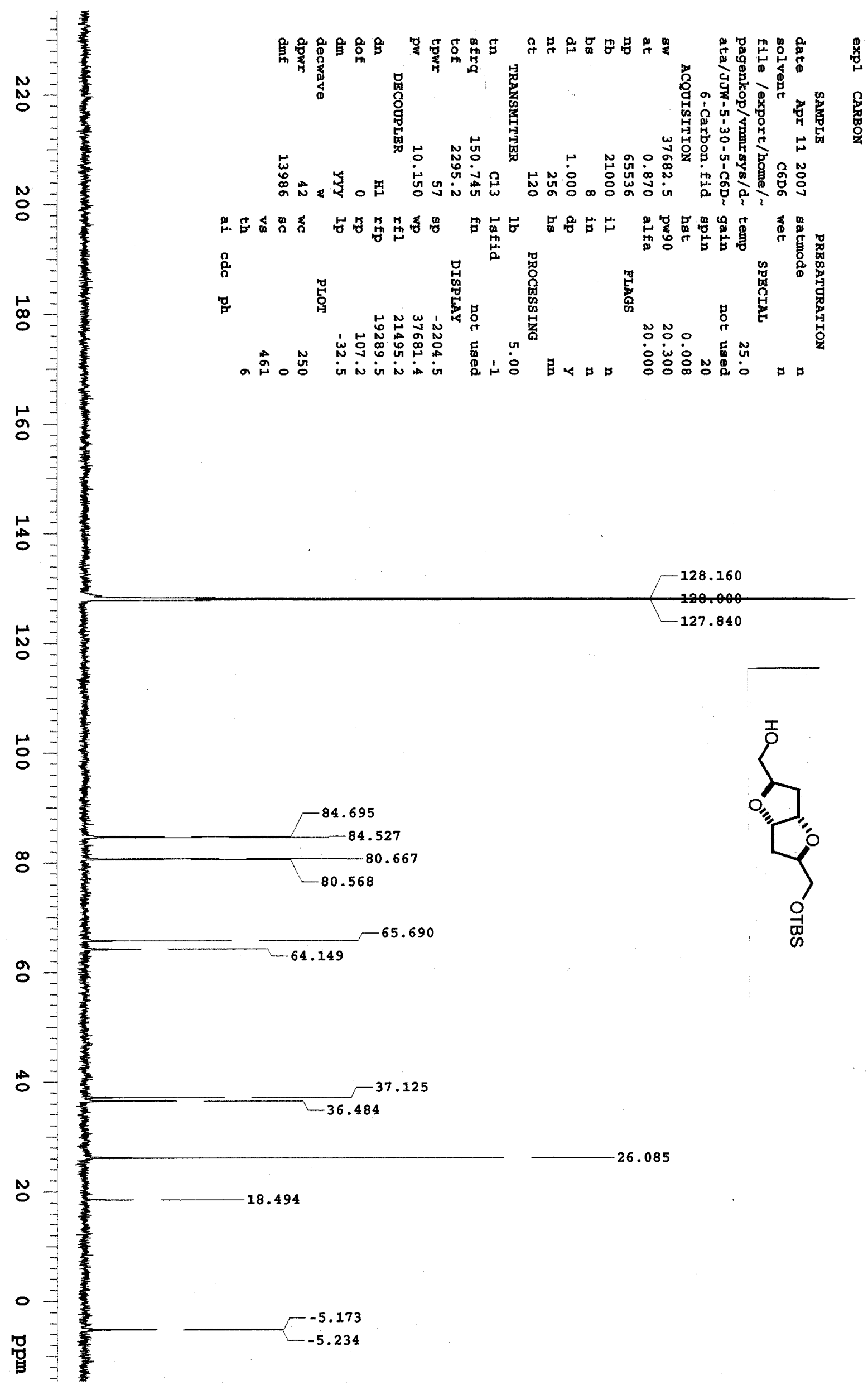




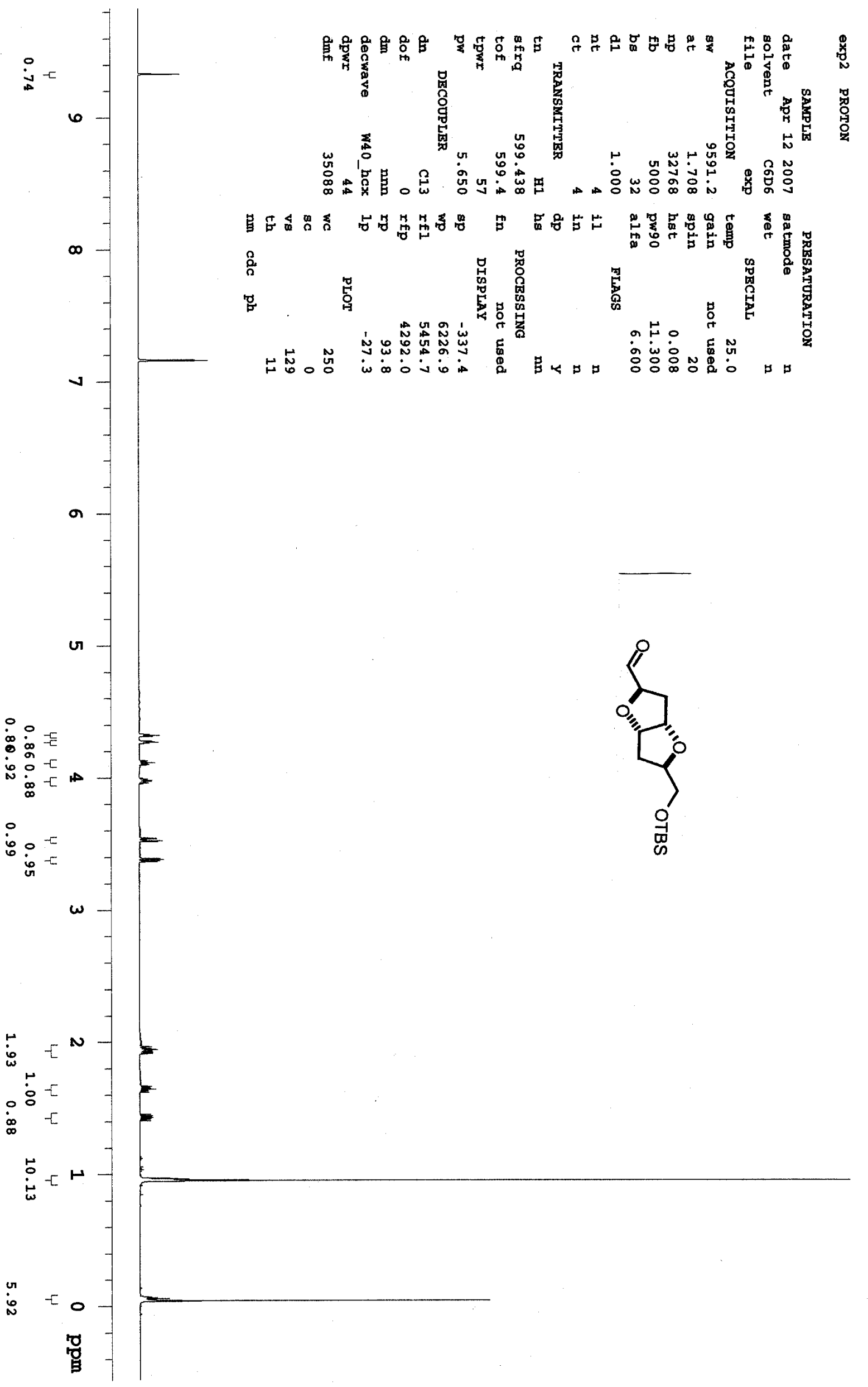




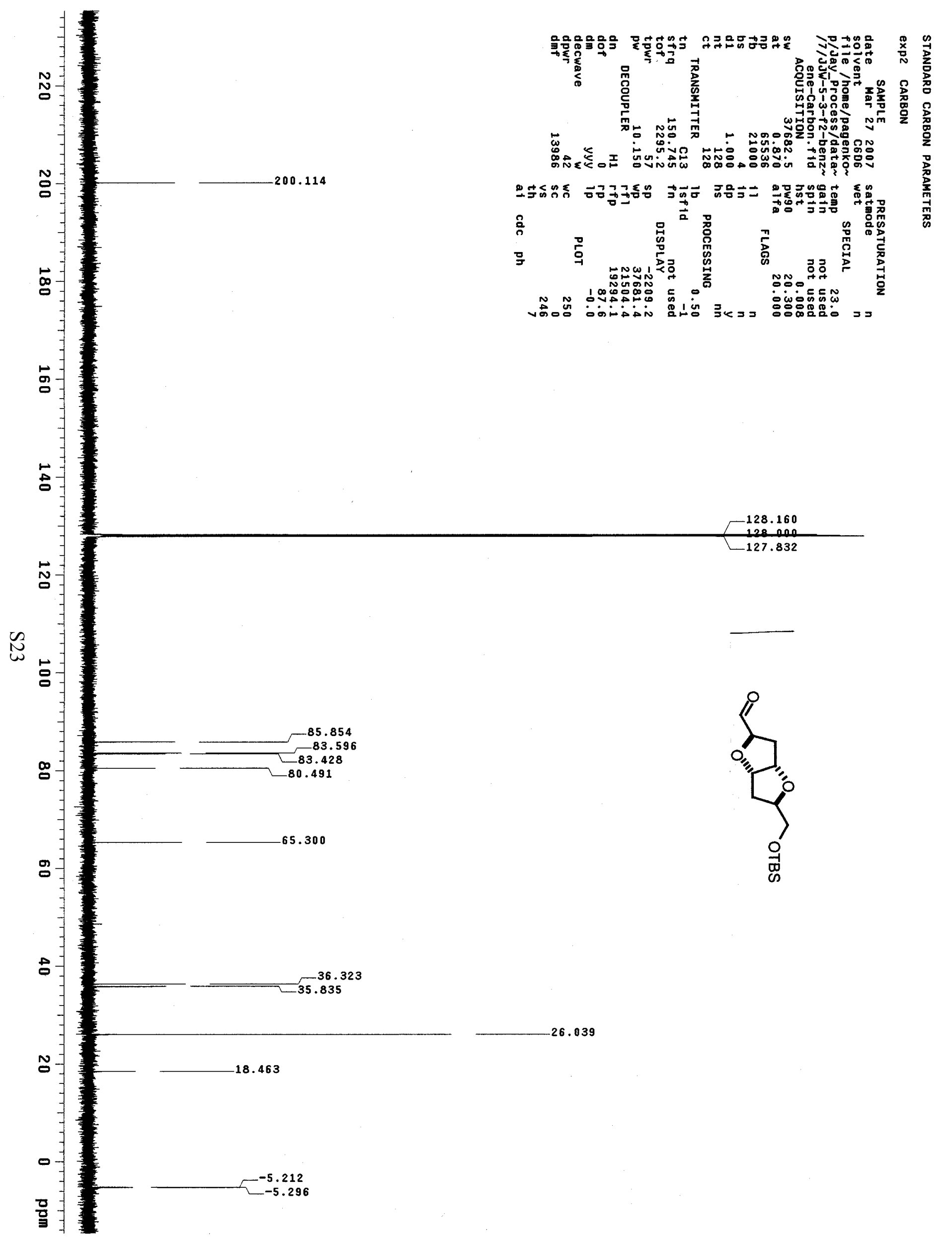




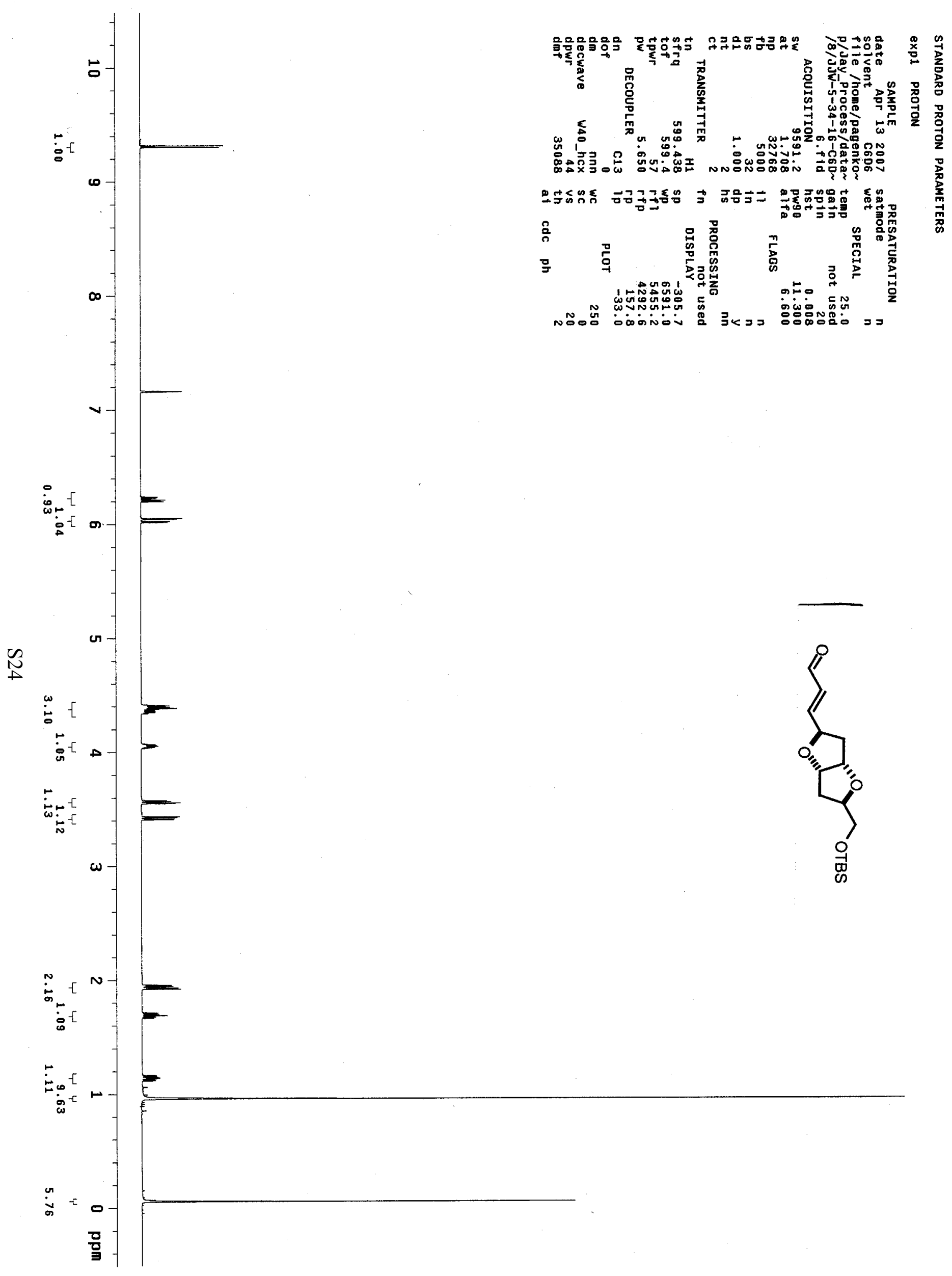




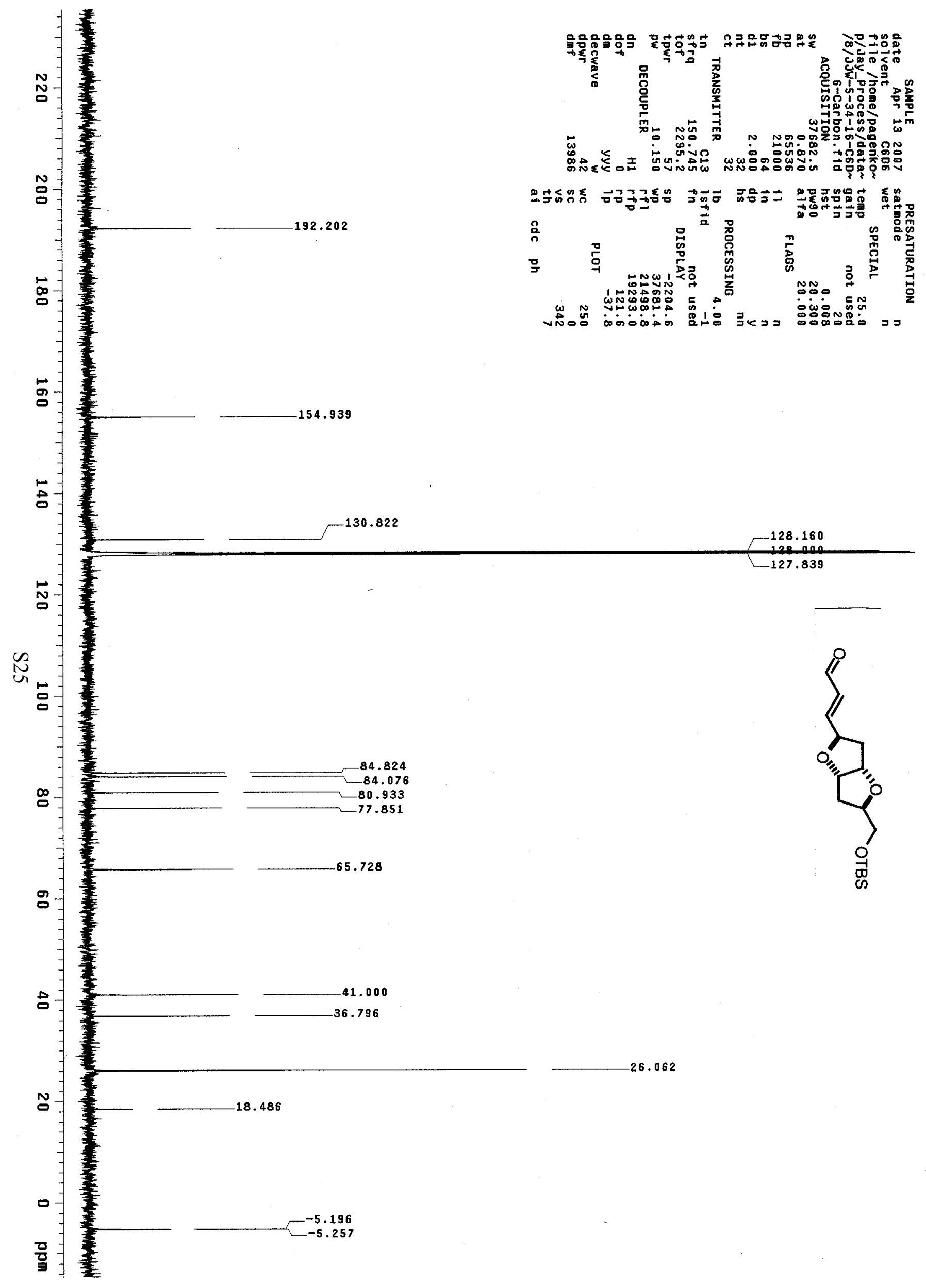




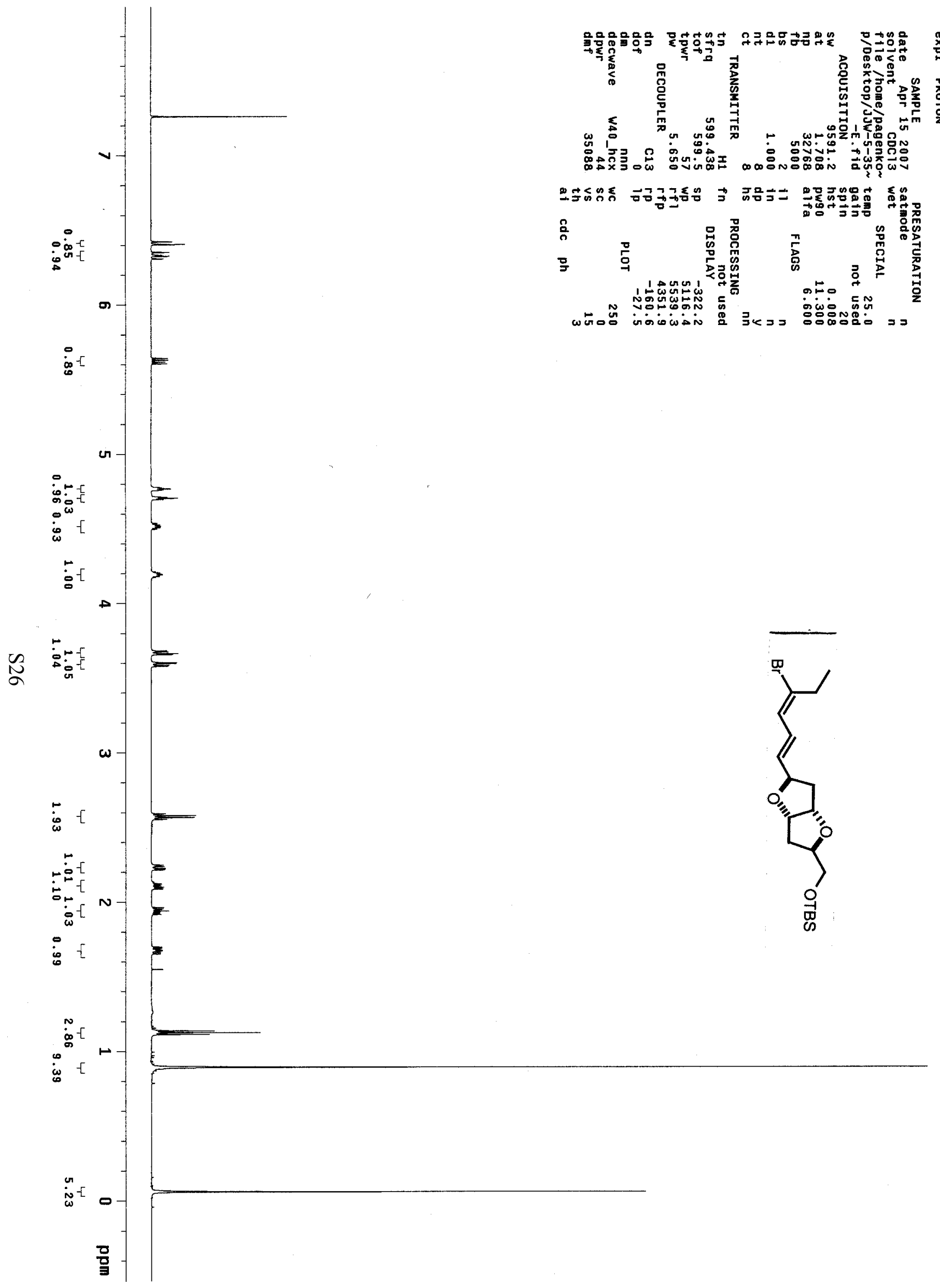




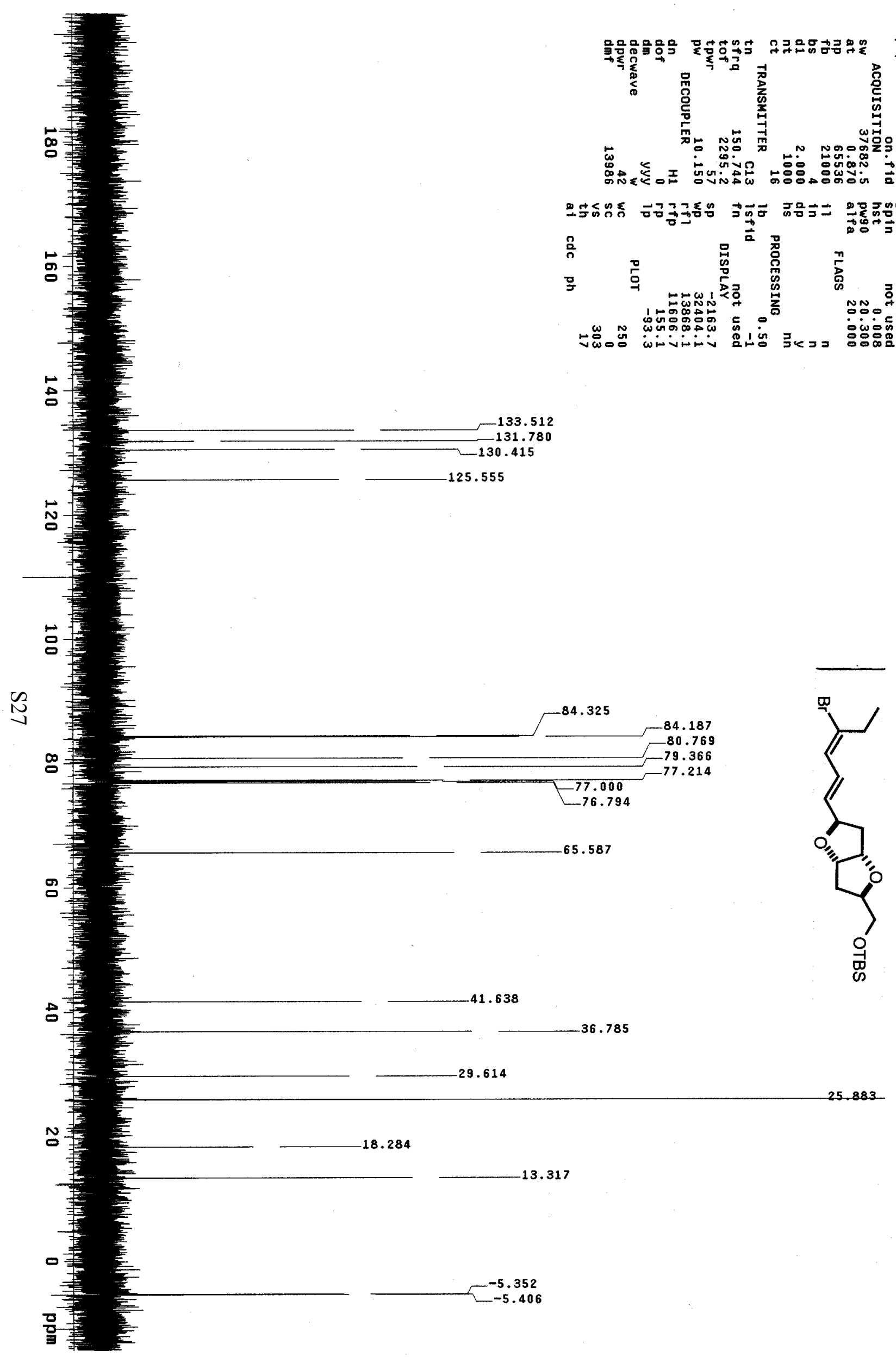




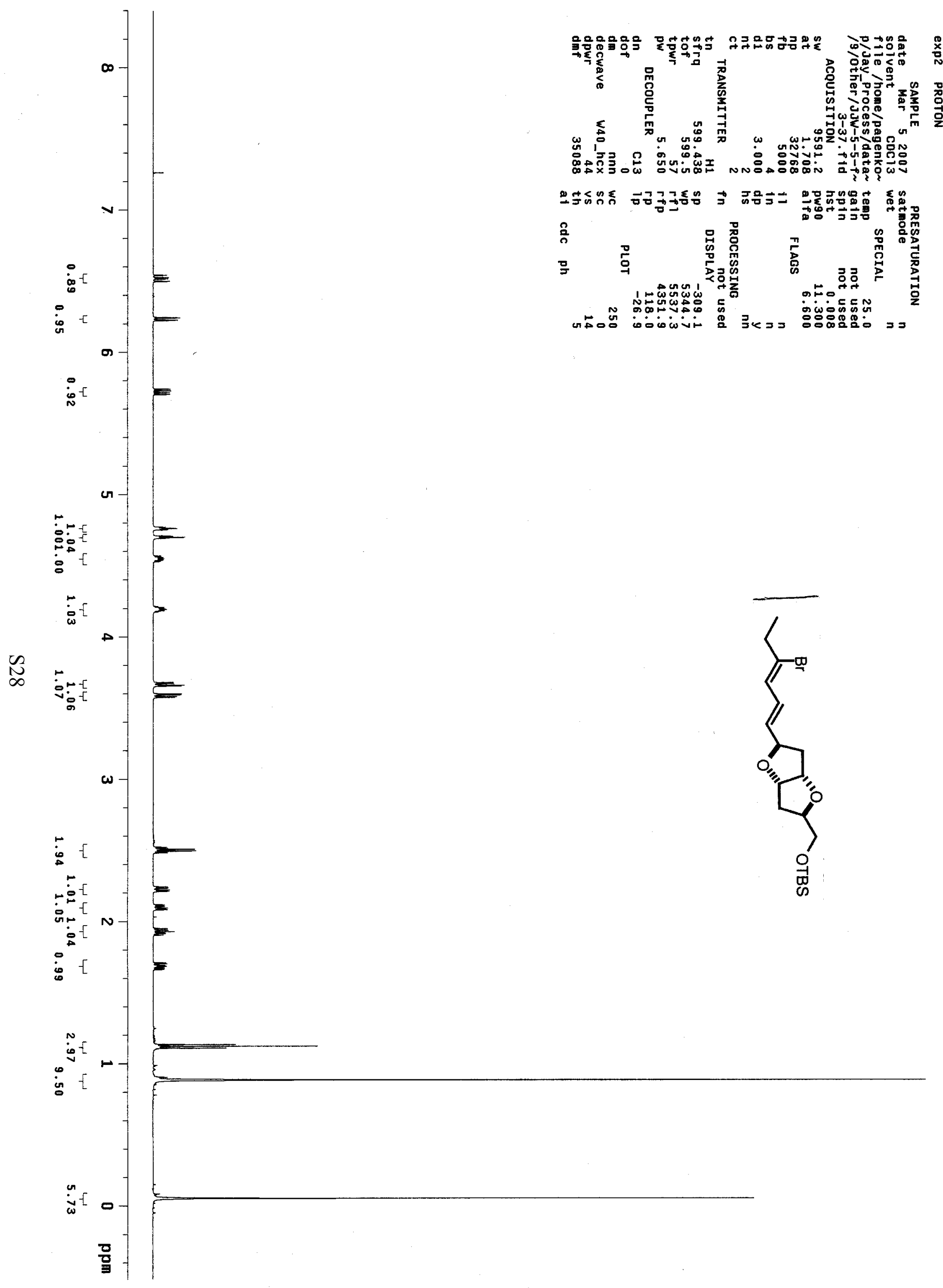




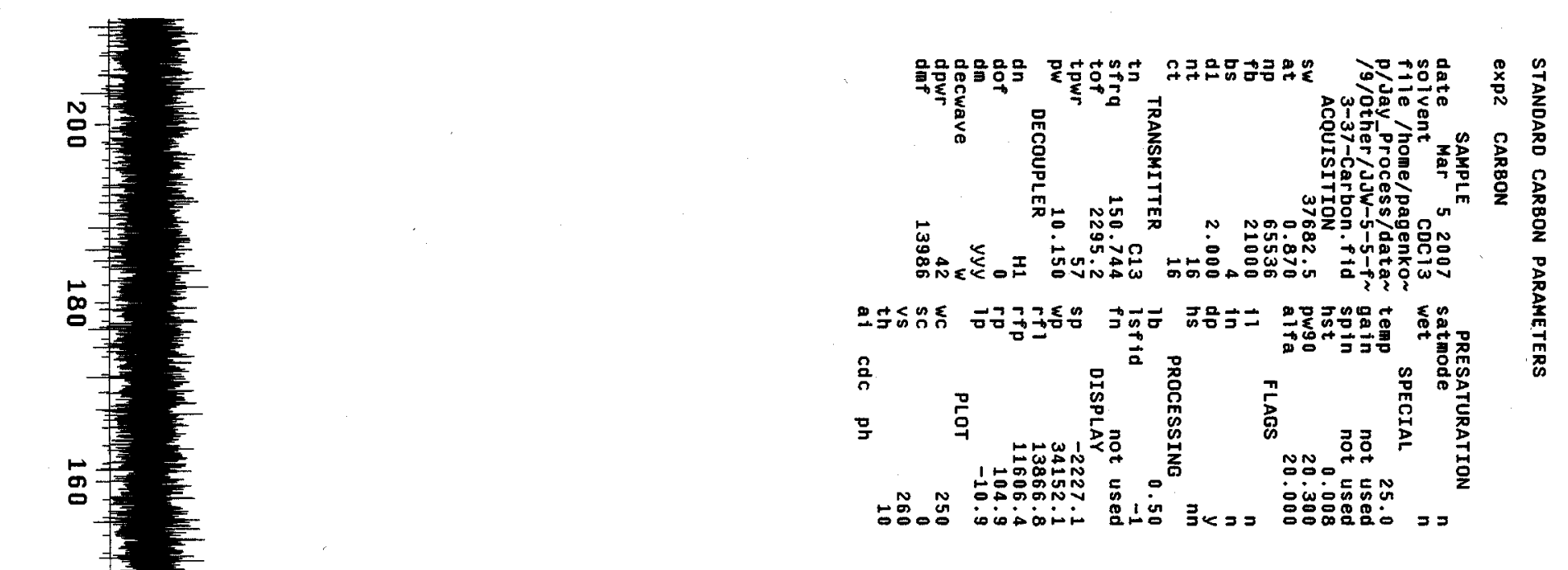

$-125.714$

Nู

응

$\stackrel{\bullet}{\circ}$

合

134.541

$-129.315$

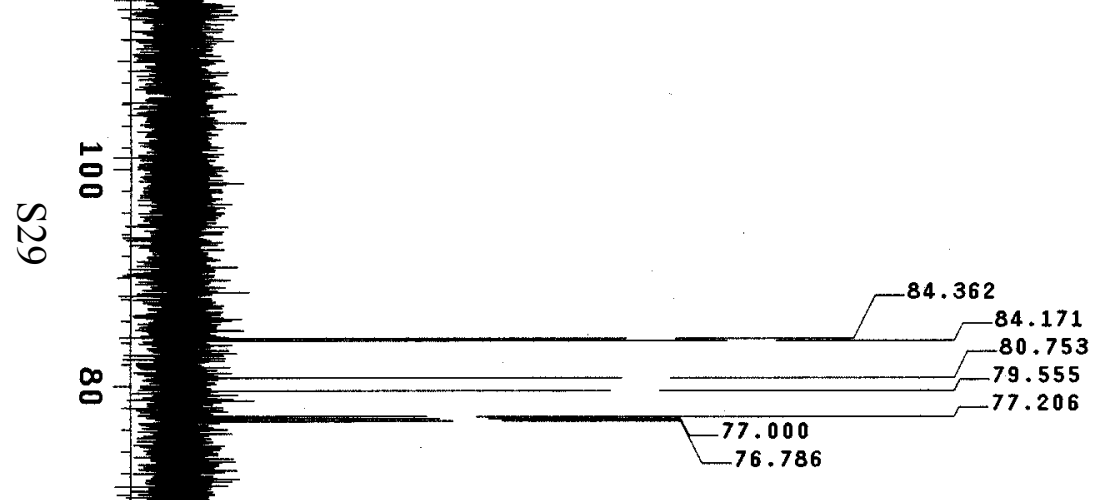

65.578

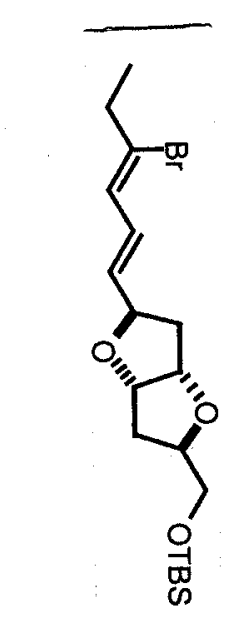

g

$-41.599$

吕
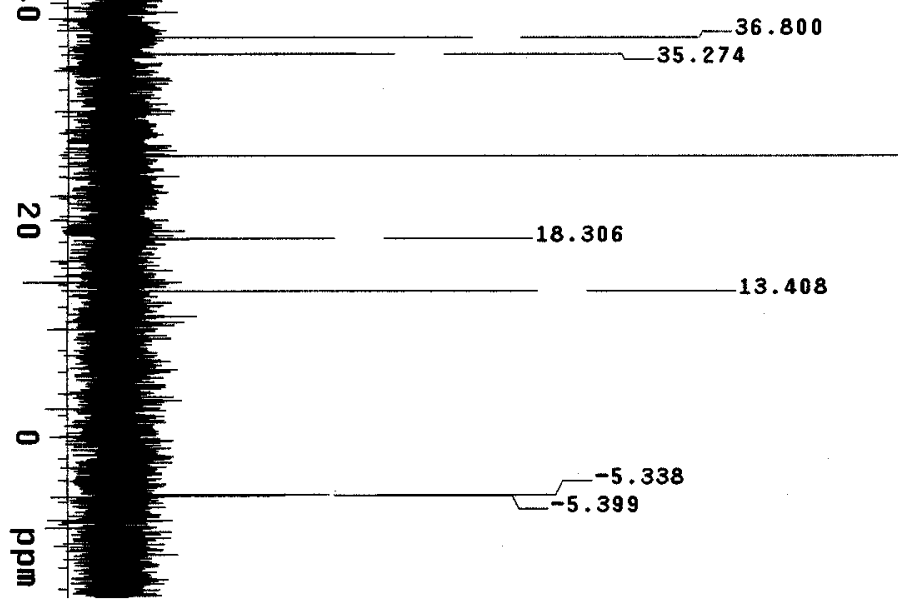

25.897 


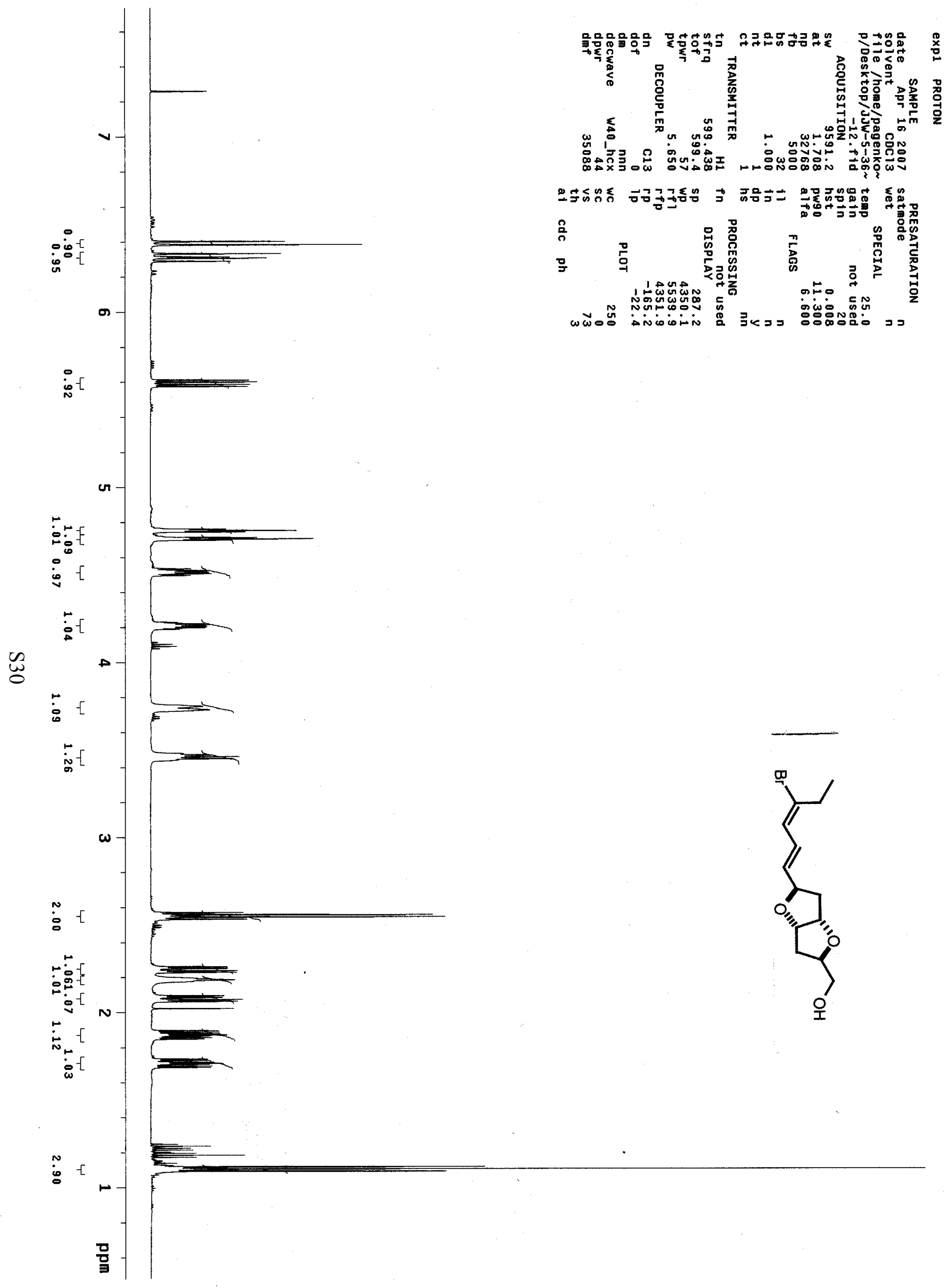




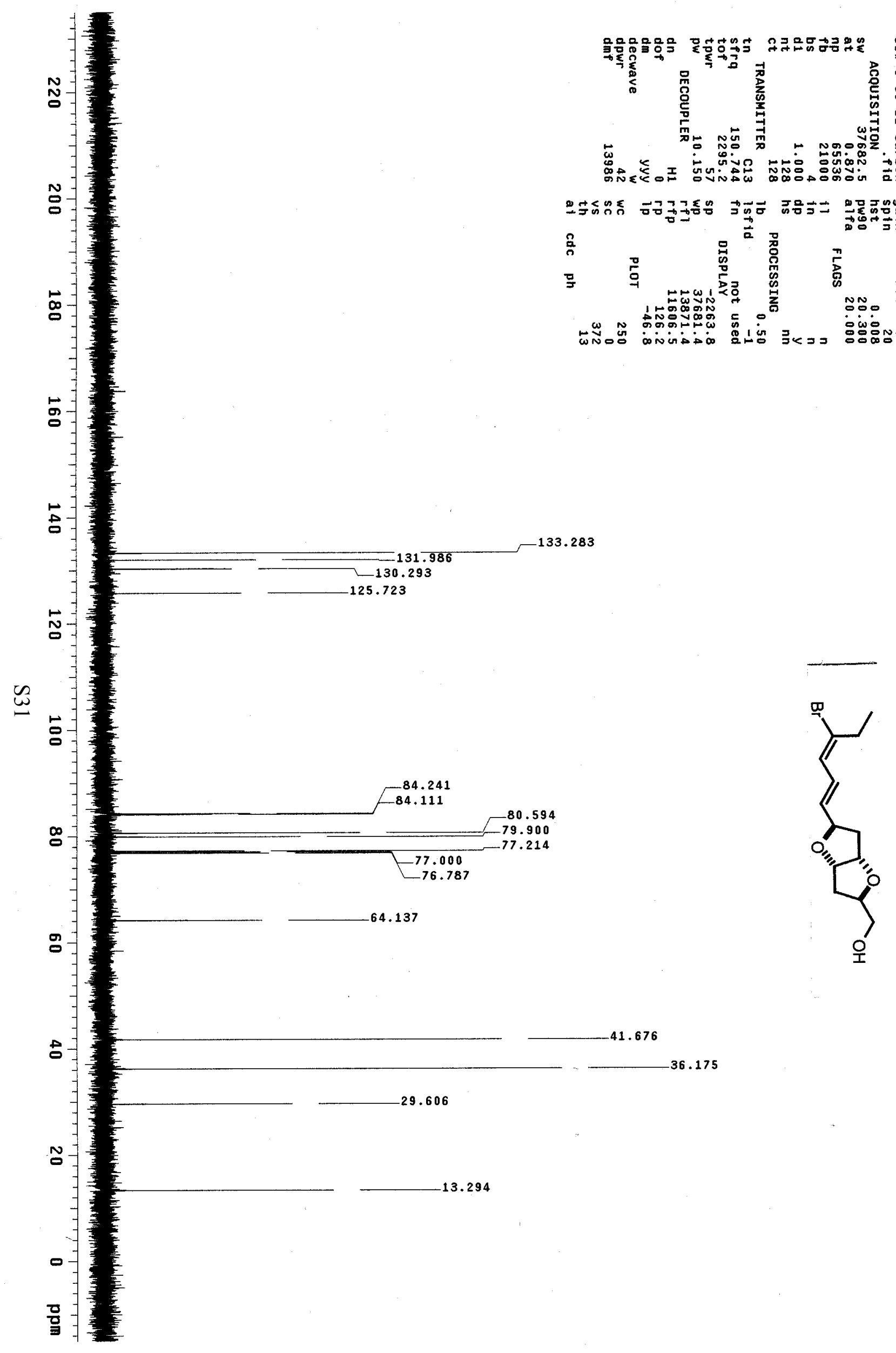




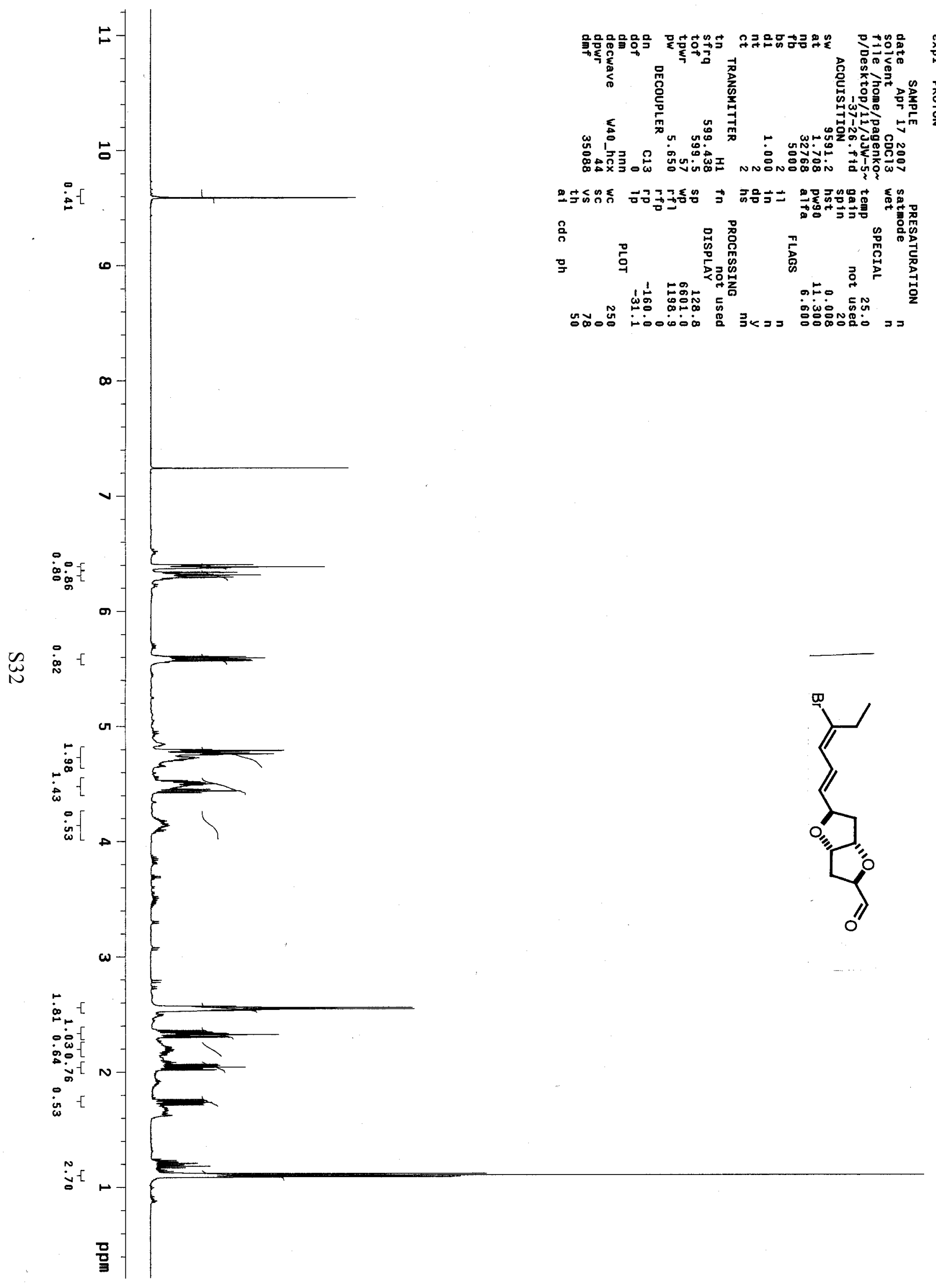




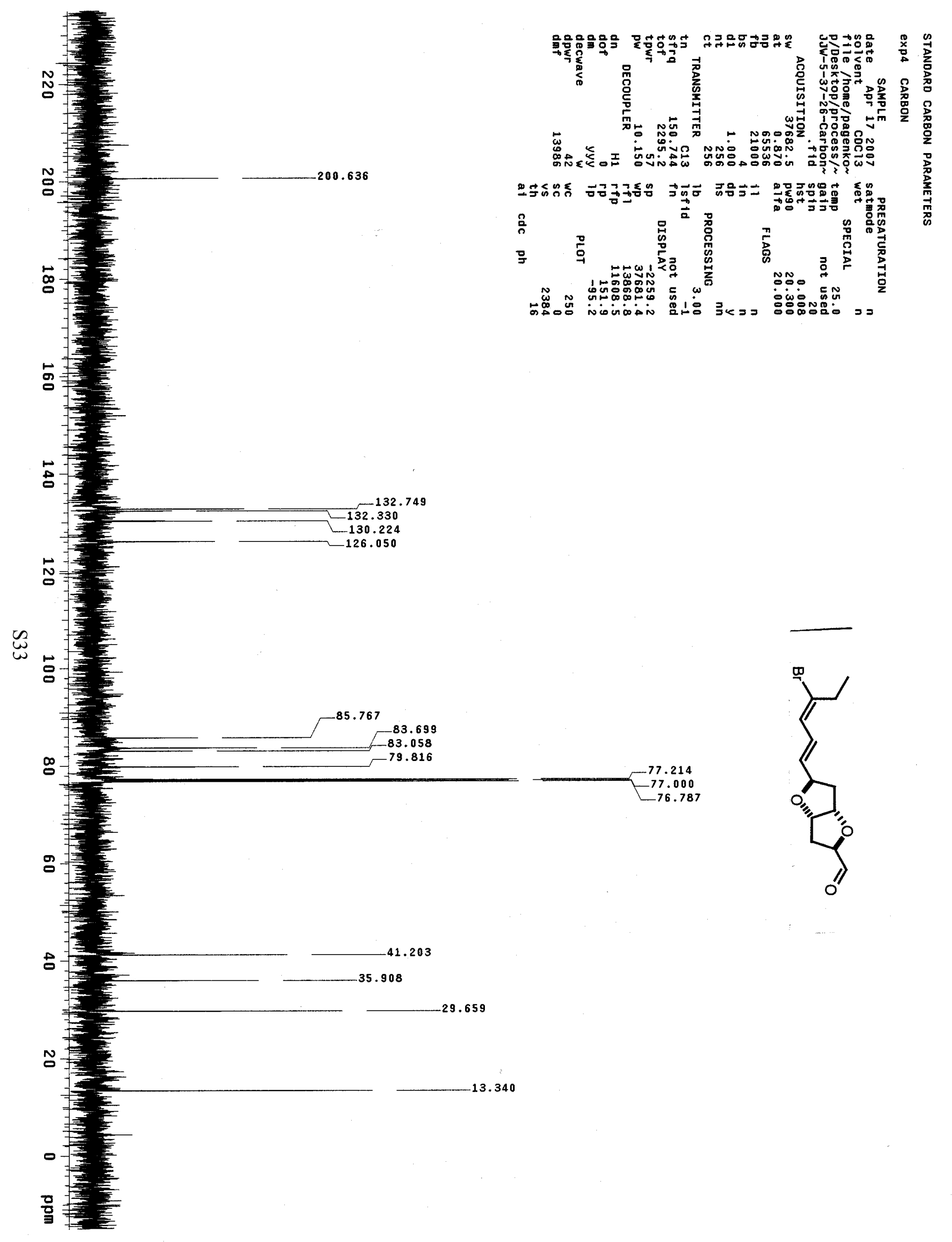




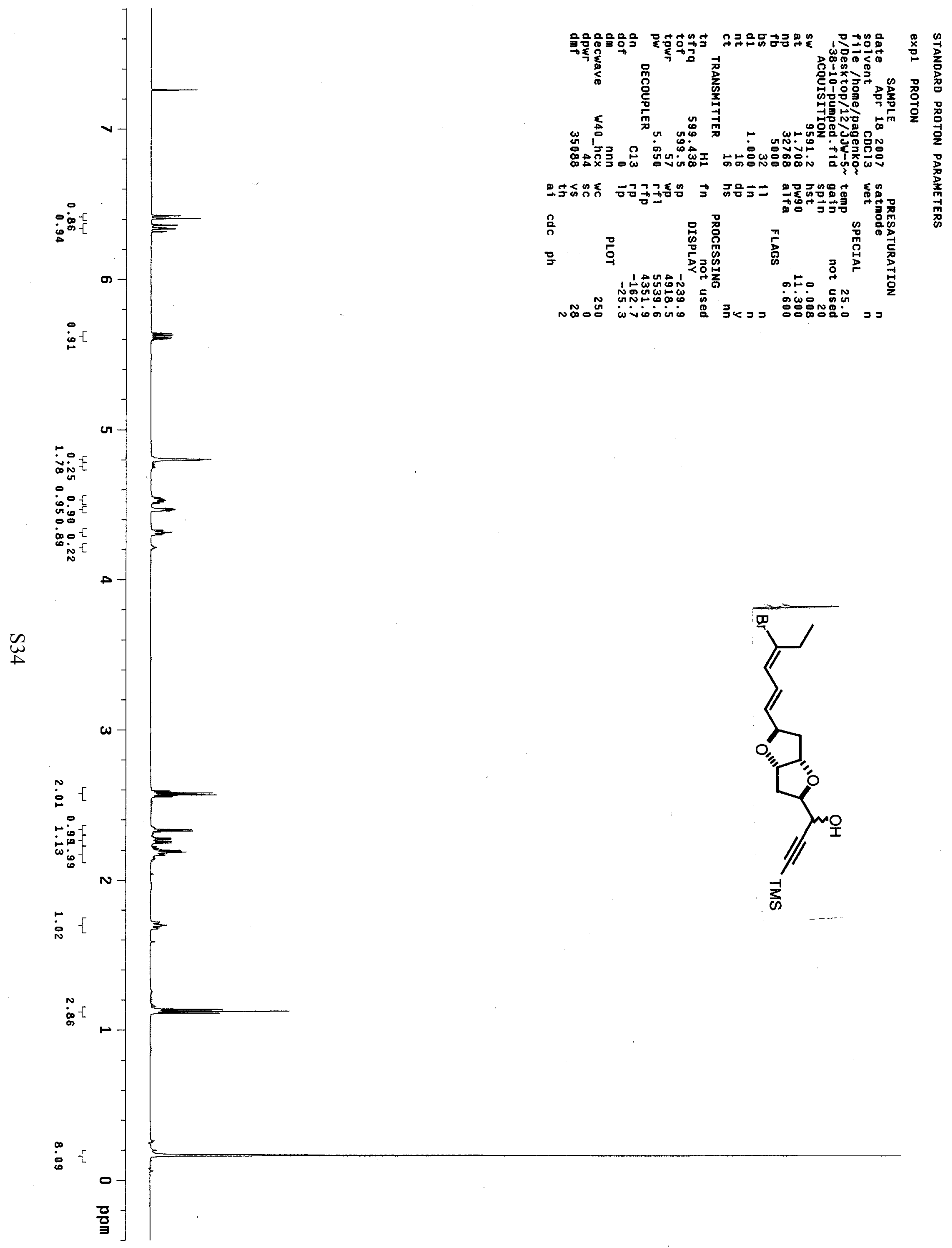




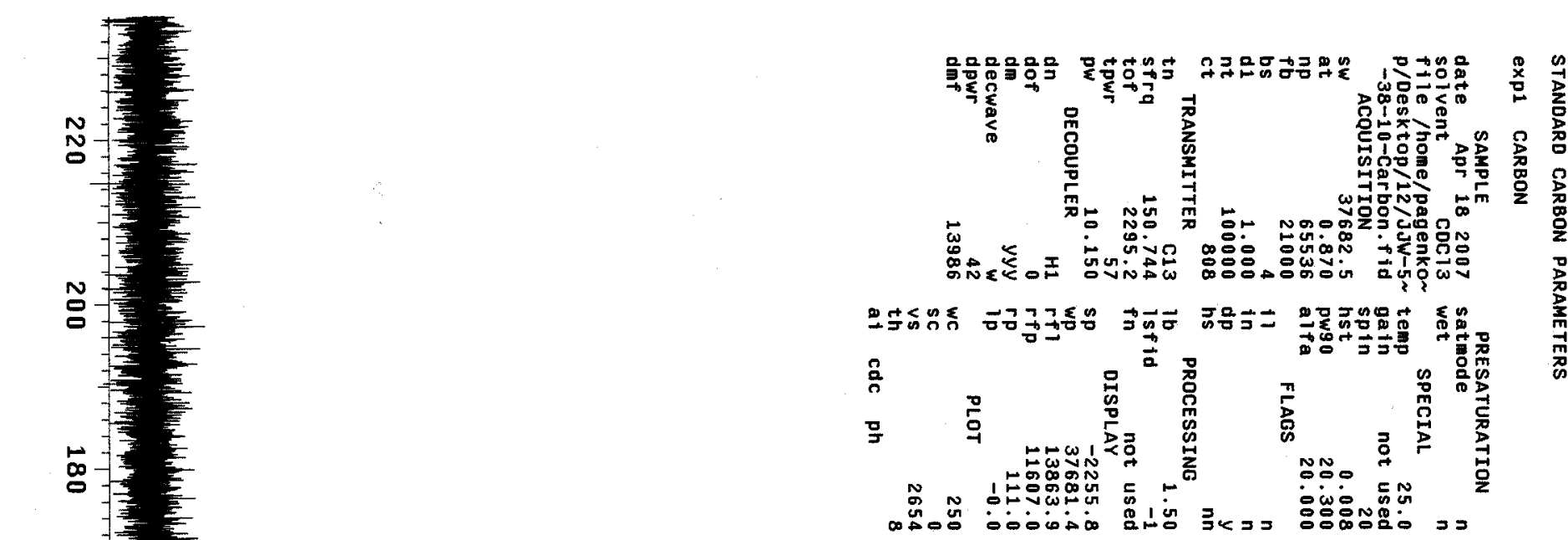

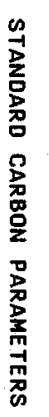

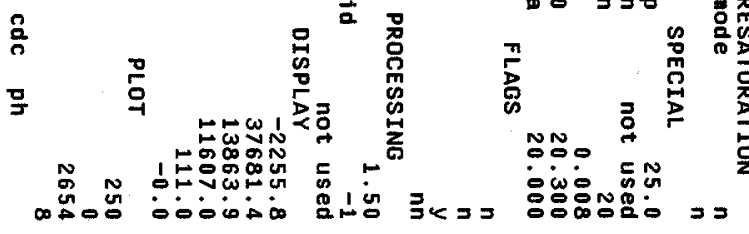

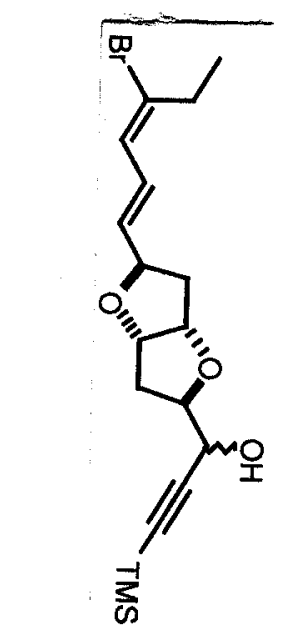

w

。

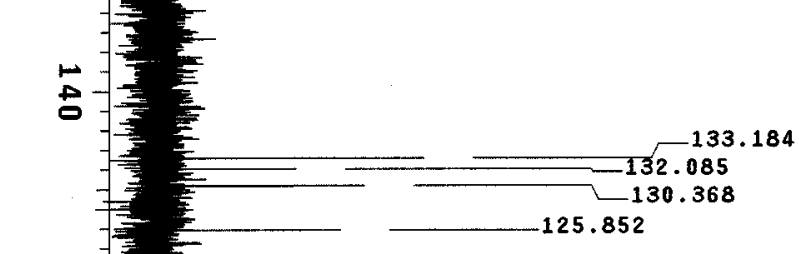

$-102.635$

음

85.438

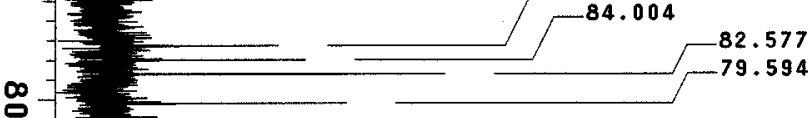

g
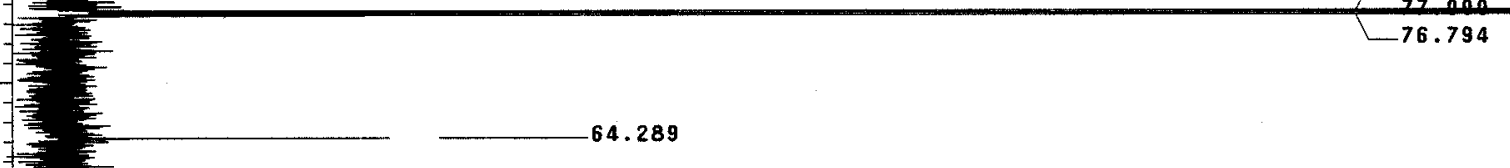

$\overrightarrow{0}$

-

.

N

ㄴ.

$-13.355$

$-41.584$

29.674

0 


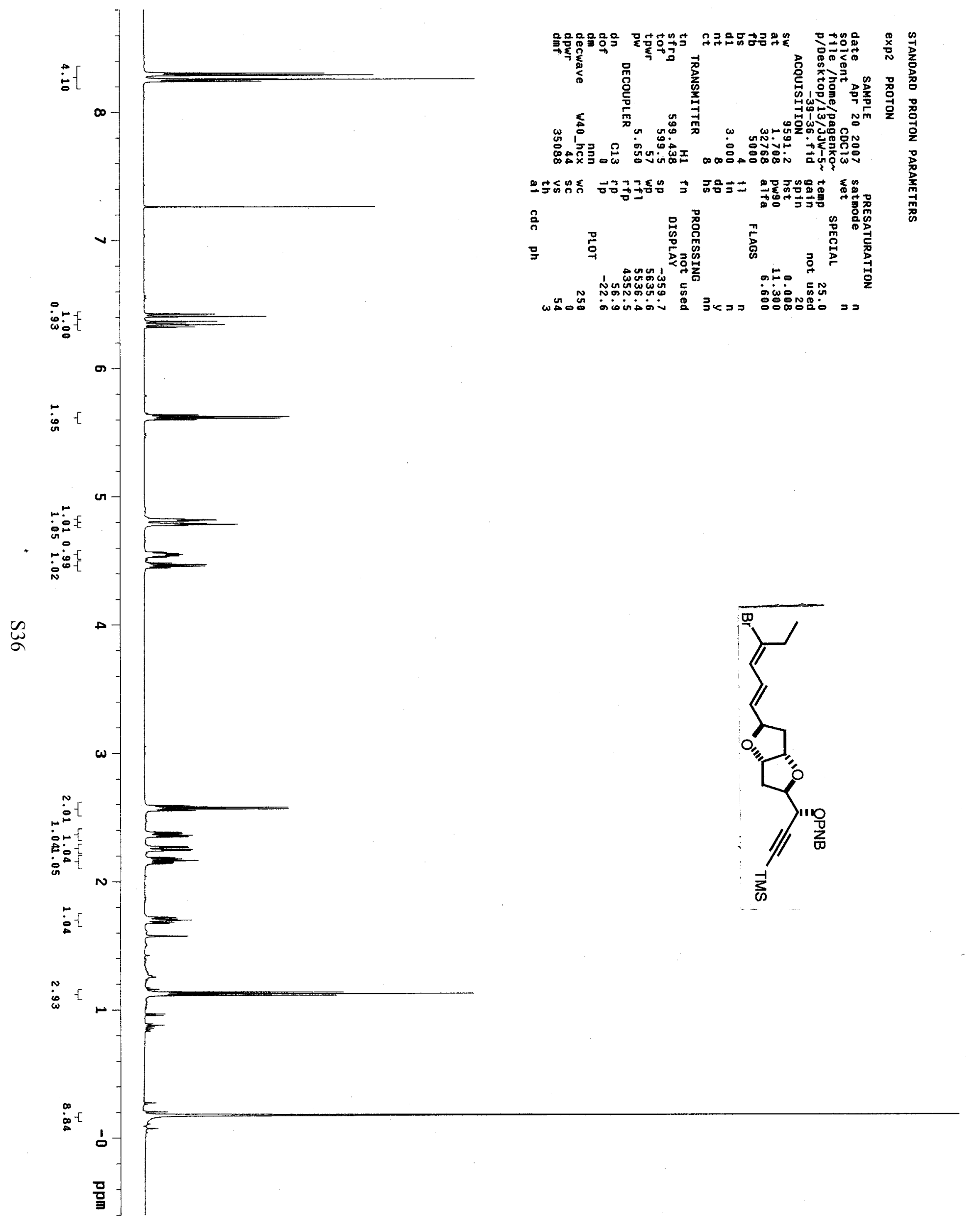




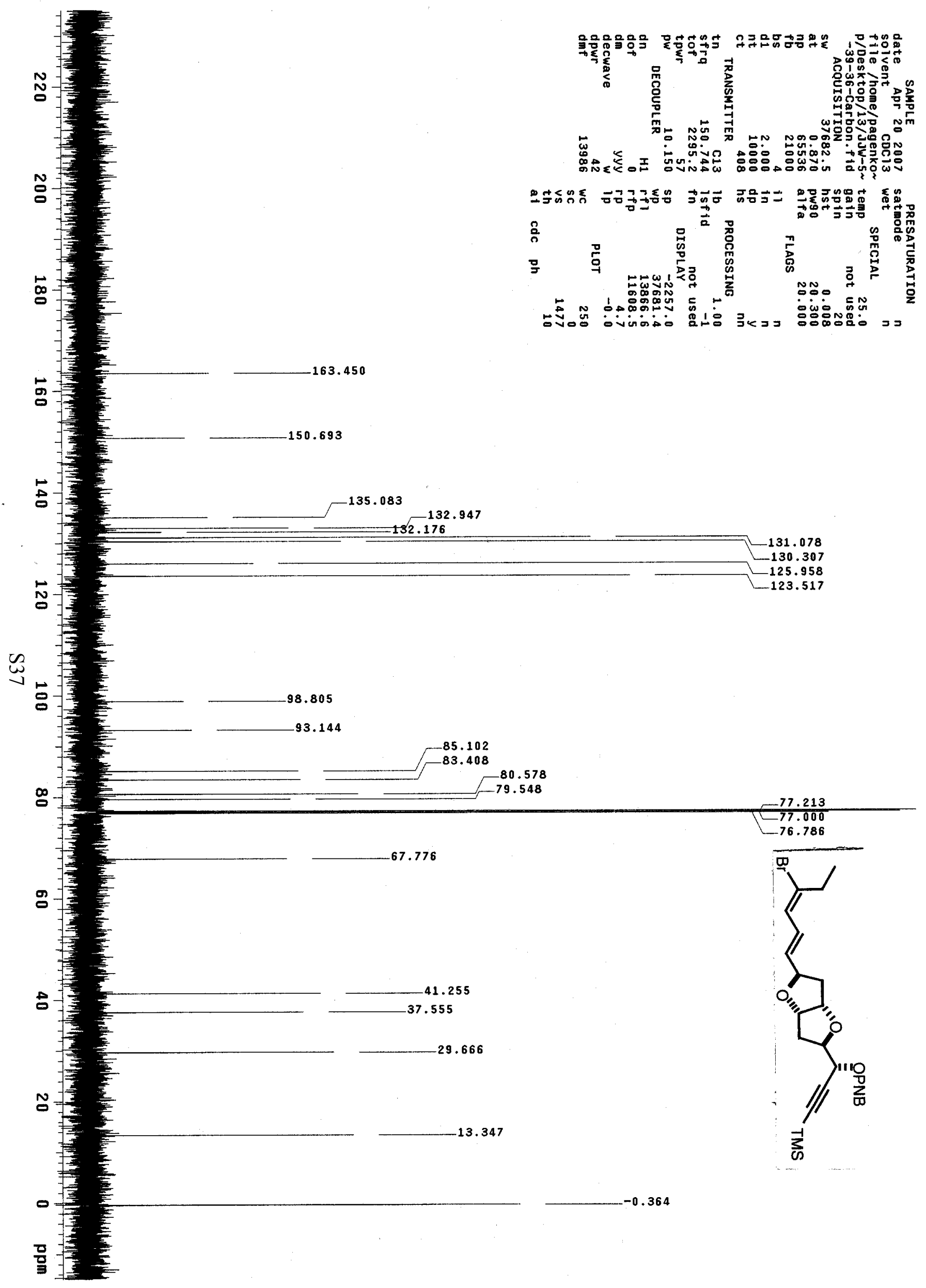




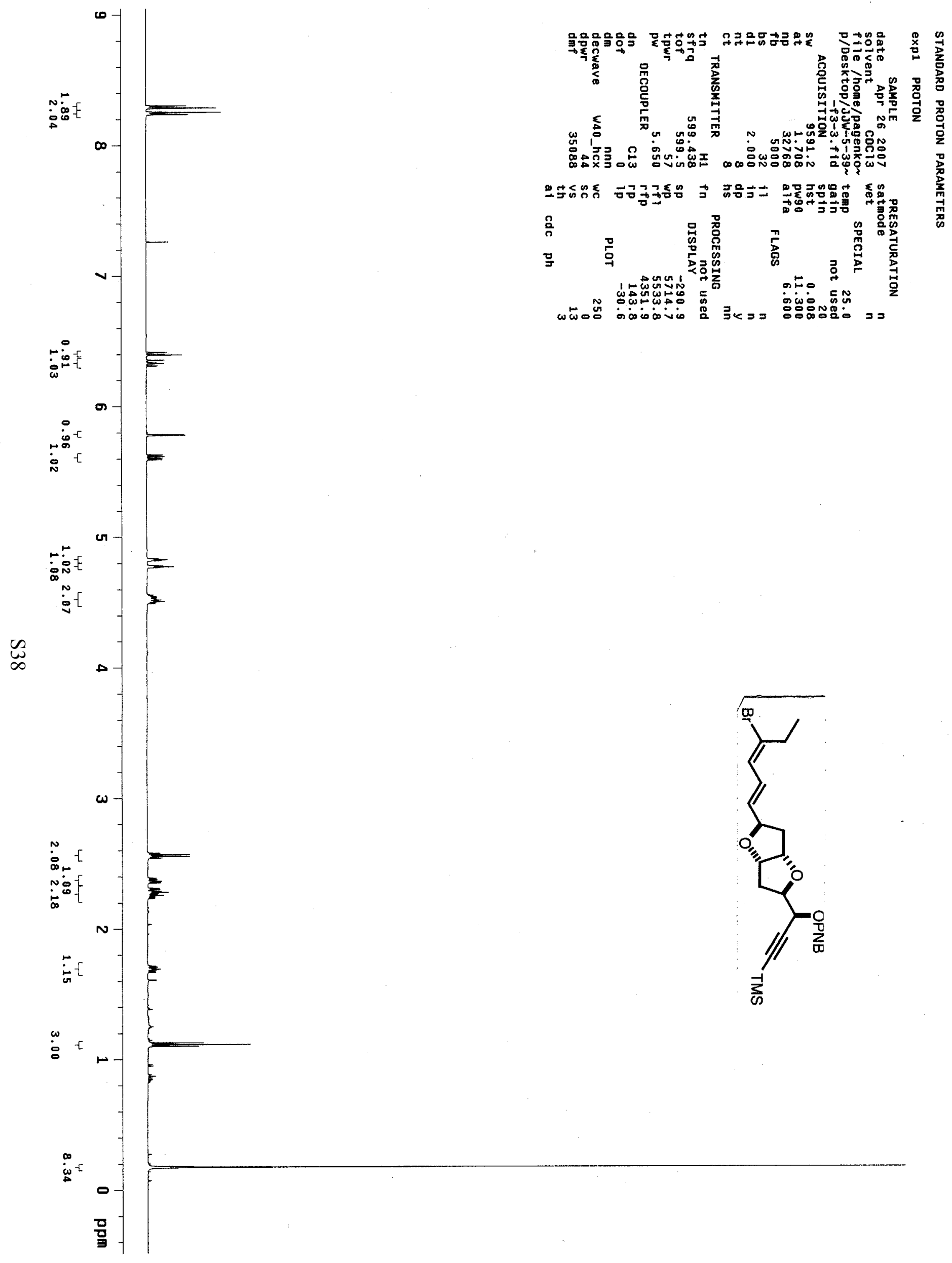




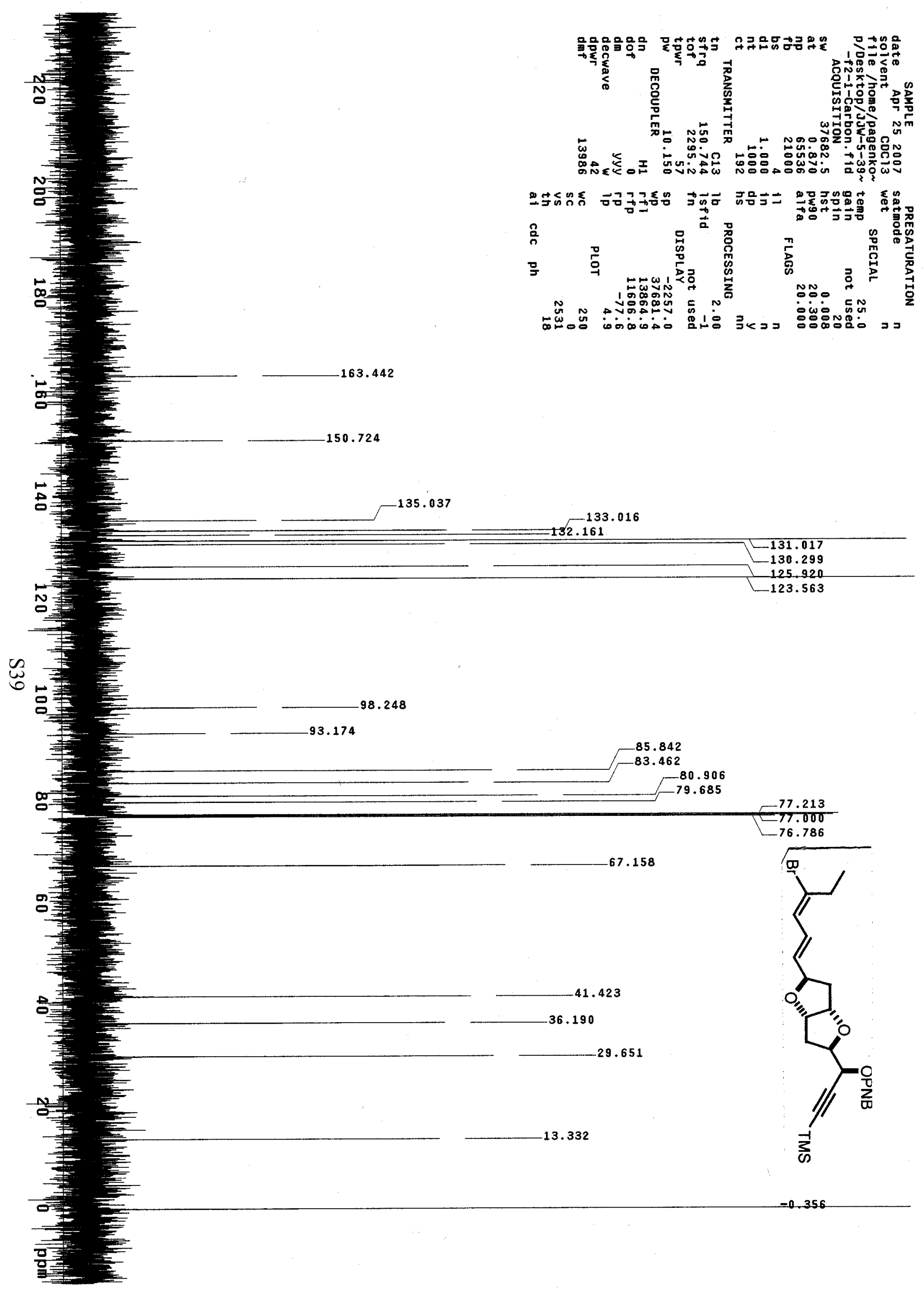




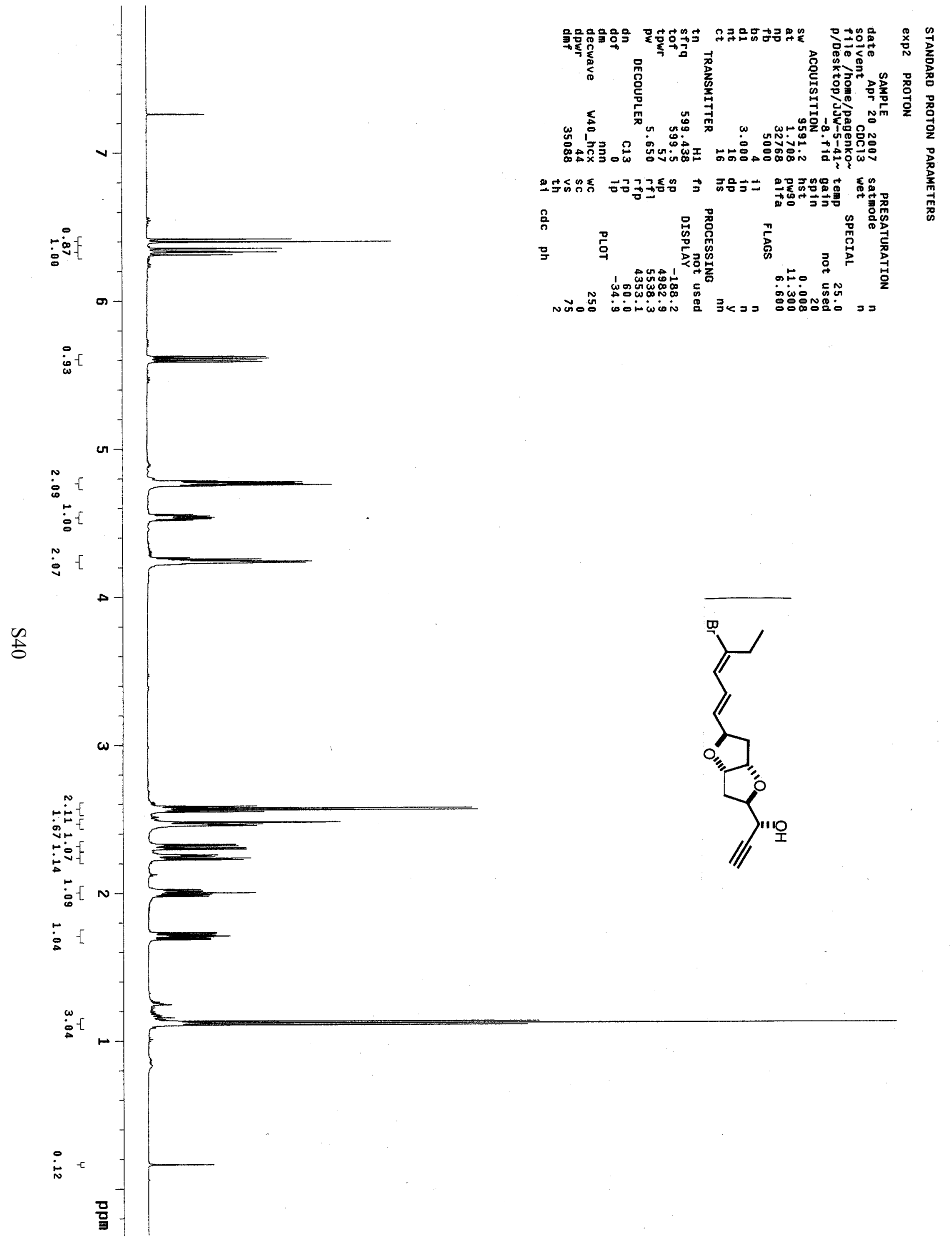




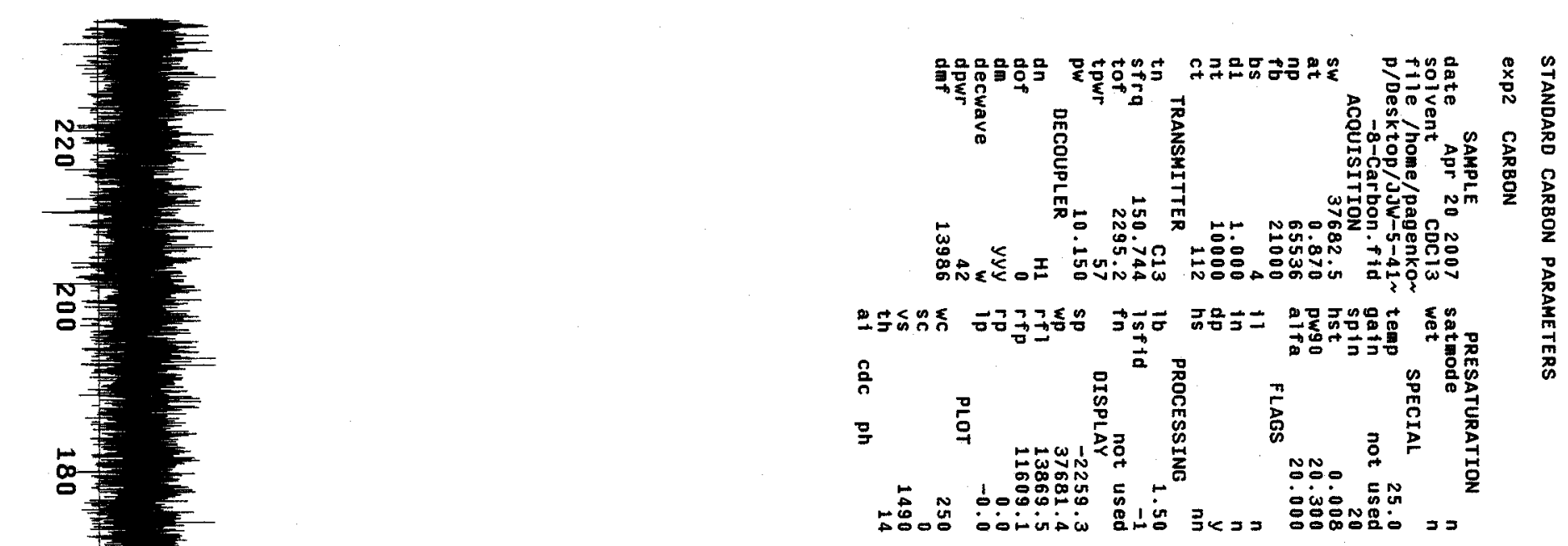

133.061

$-132.123$

$-125.928$

$\stackrel{2}{\Perp}$

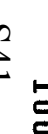

保

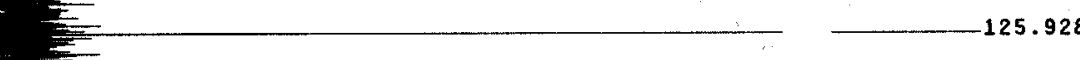

西

变
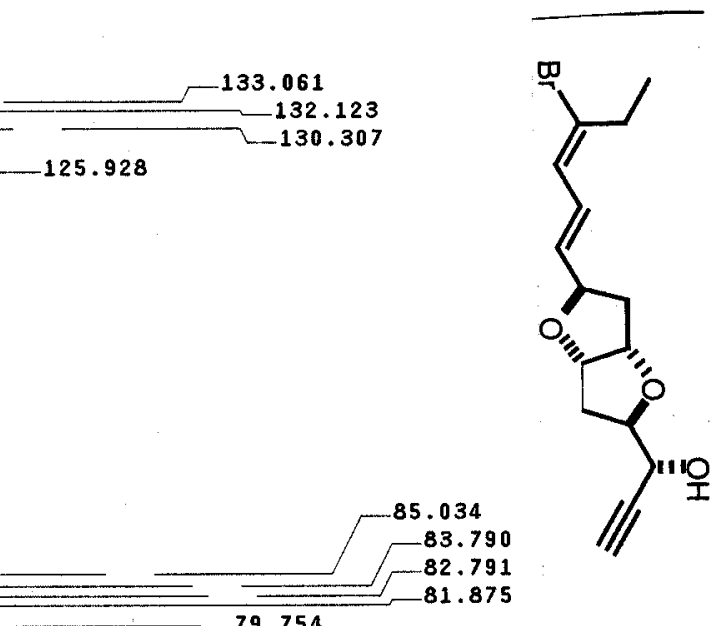

9.754

77.000

$-76.786$

64.556

g

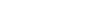

41.469

$-36.838$

$-29.651$ 


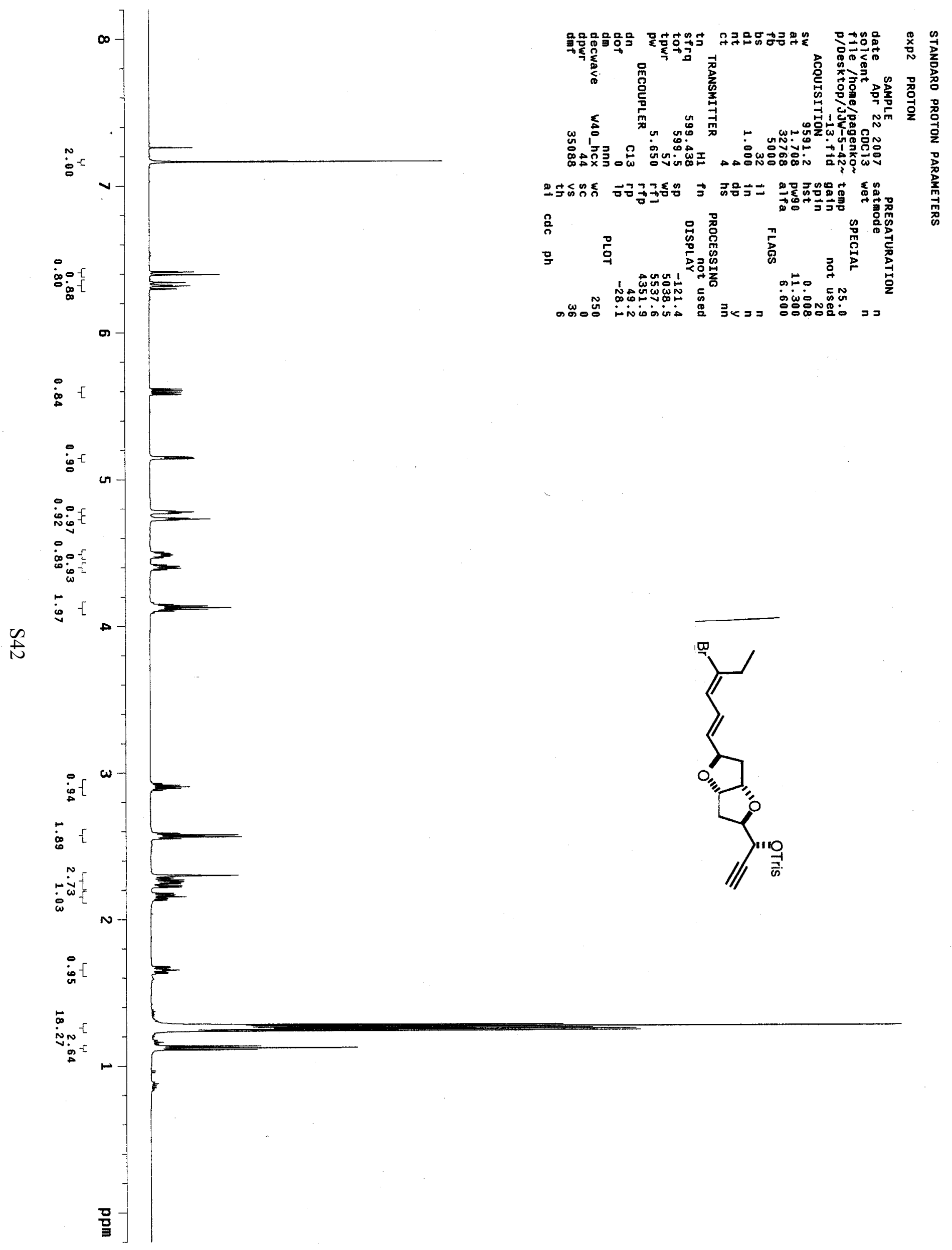




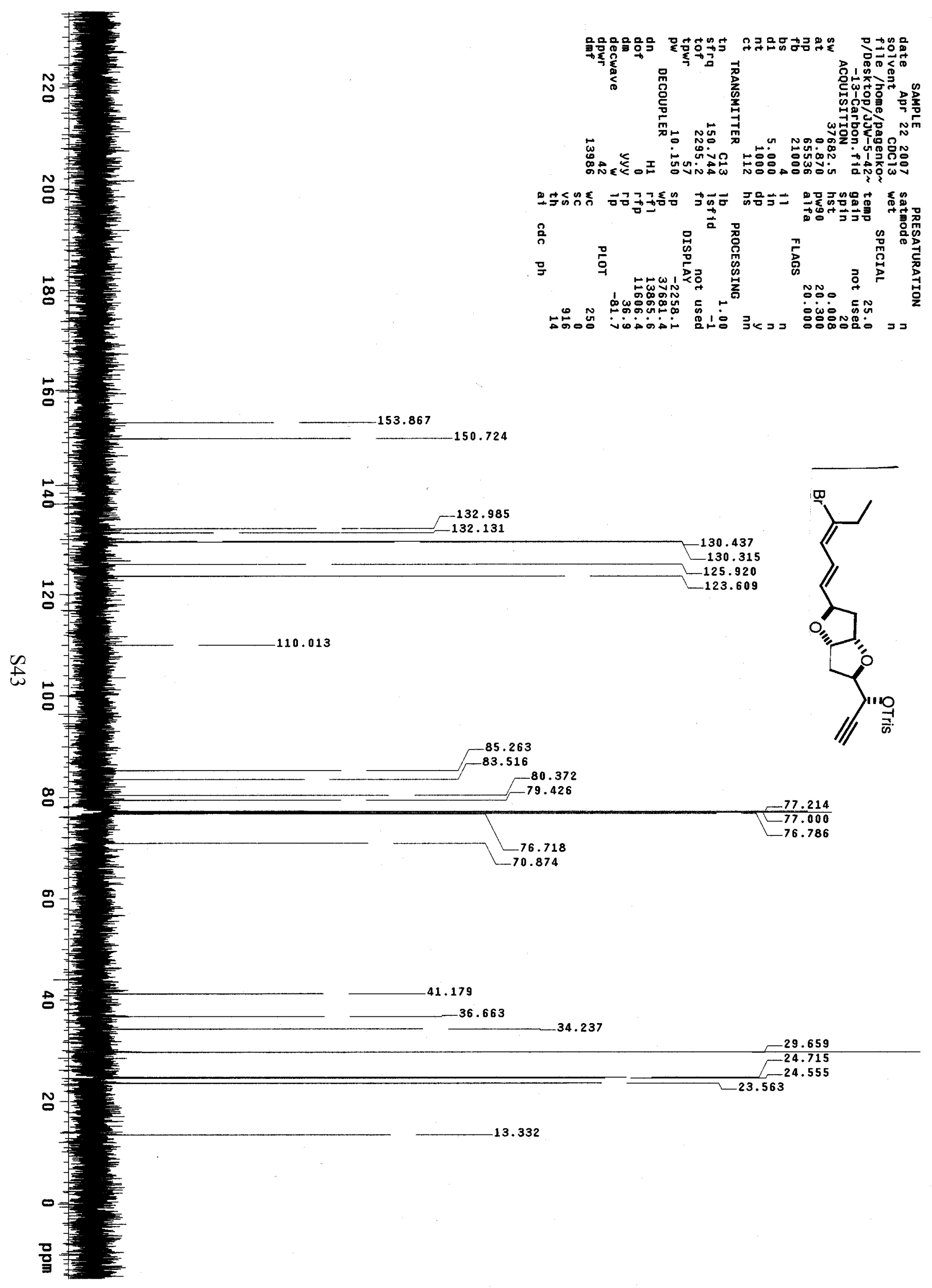




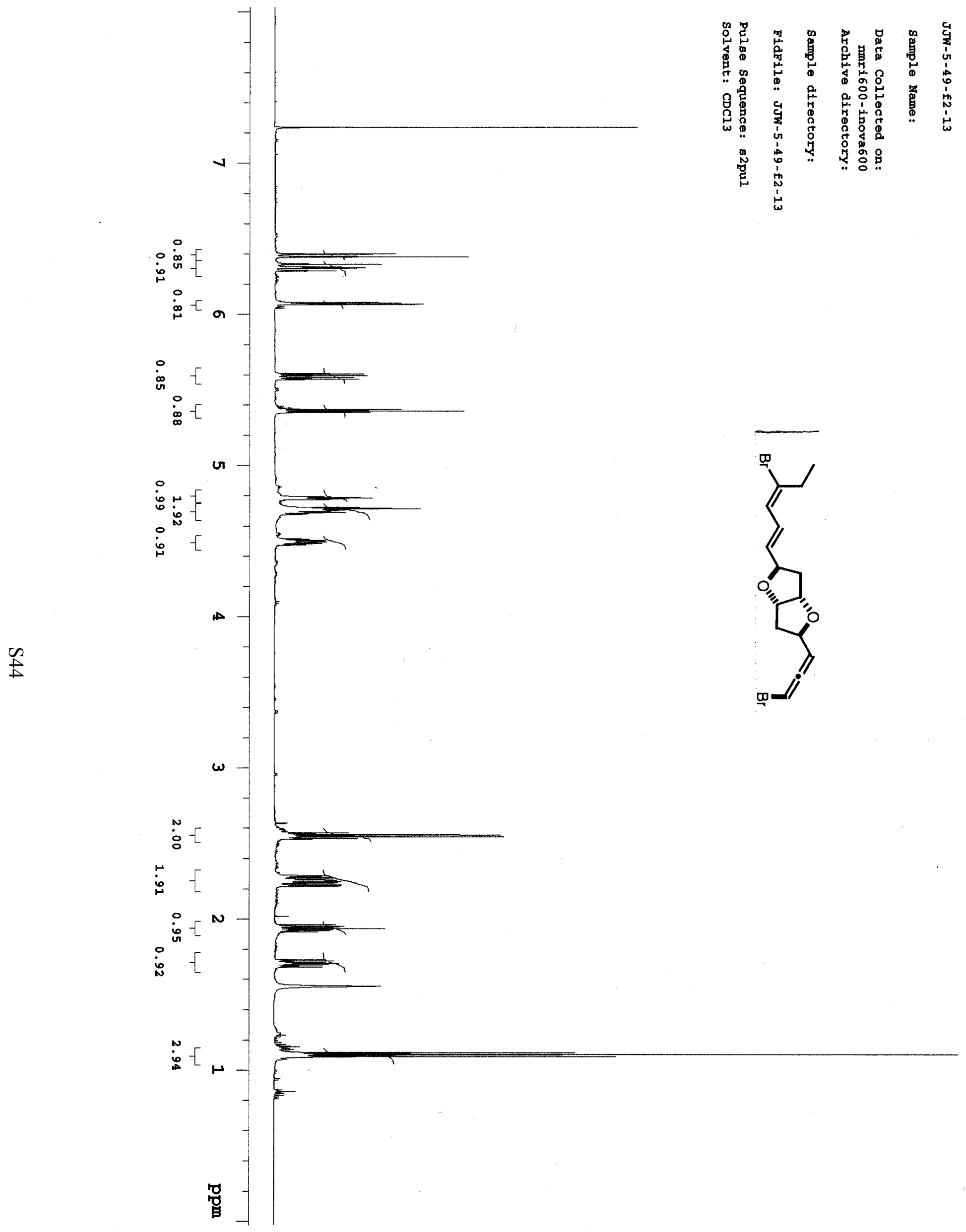




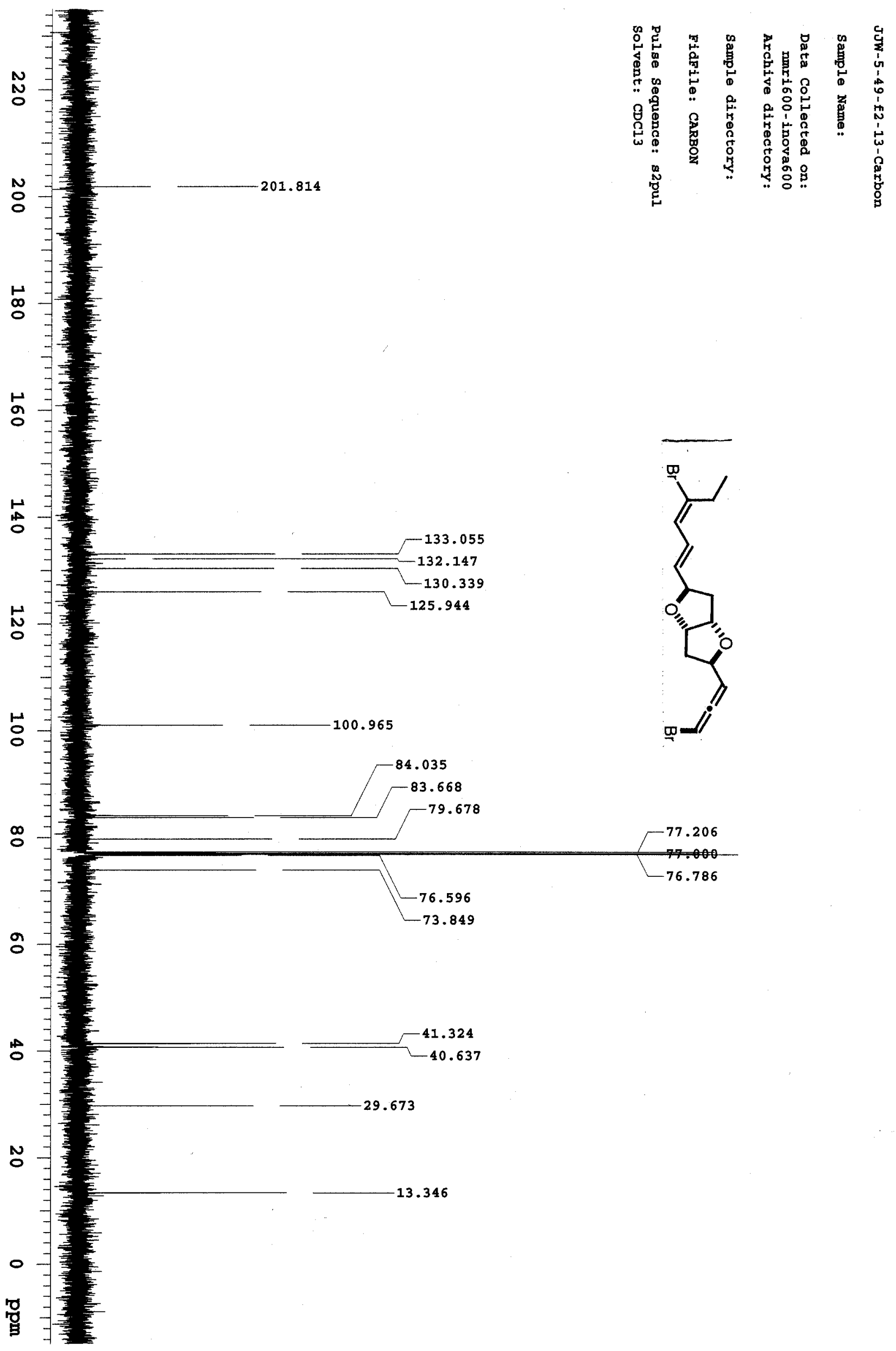




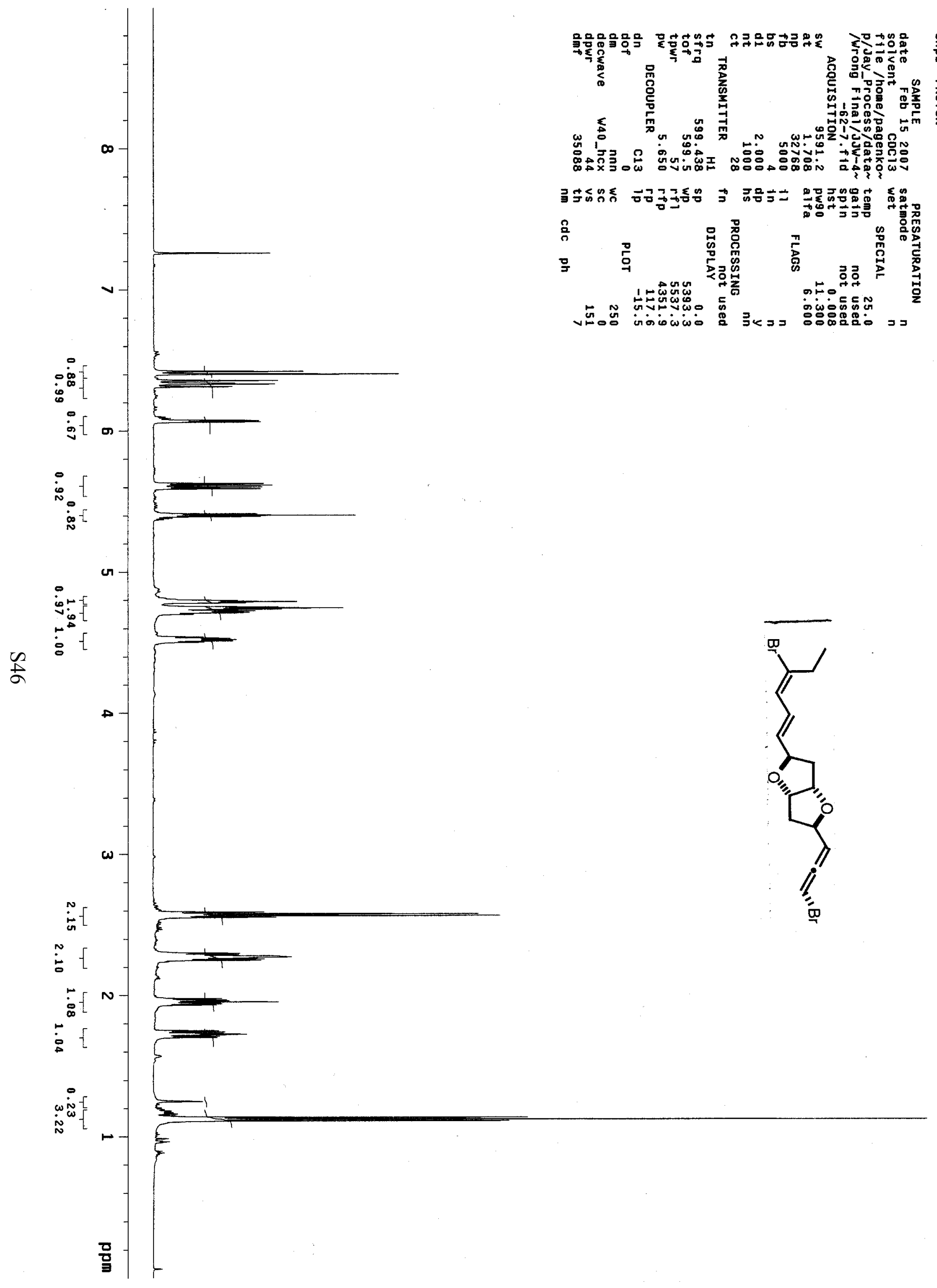




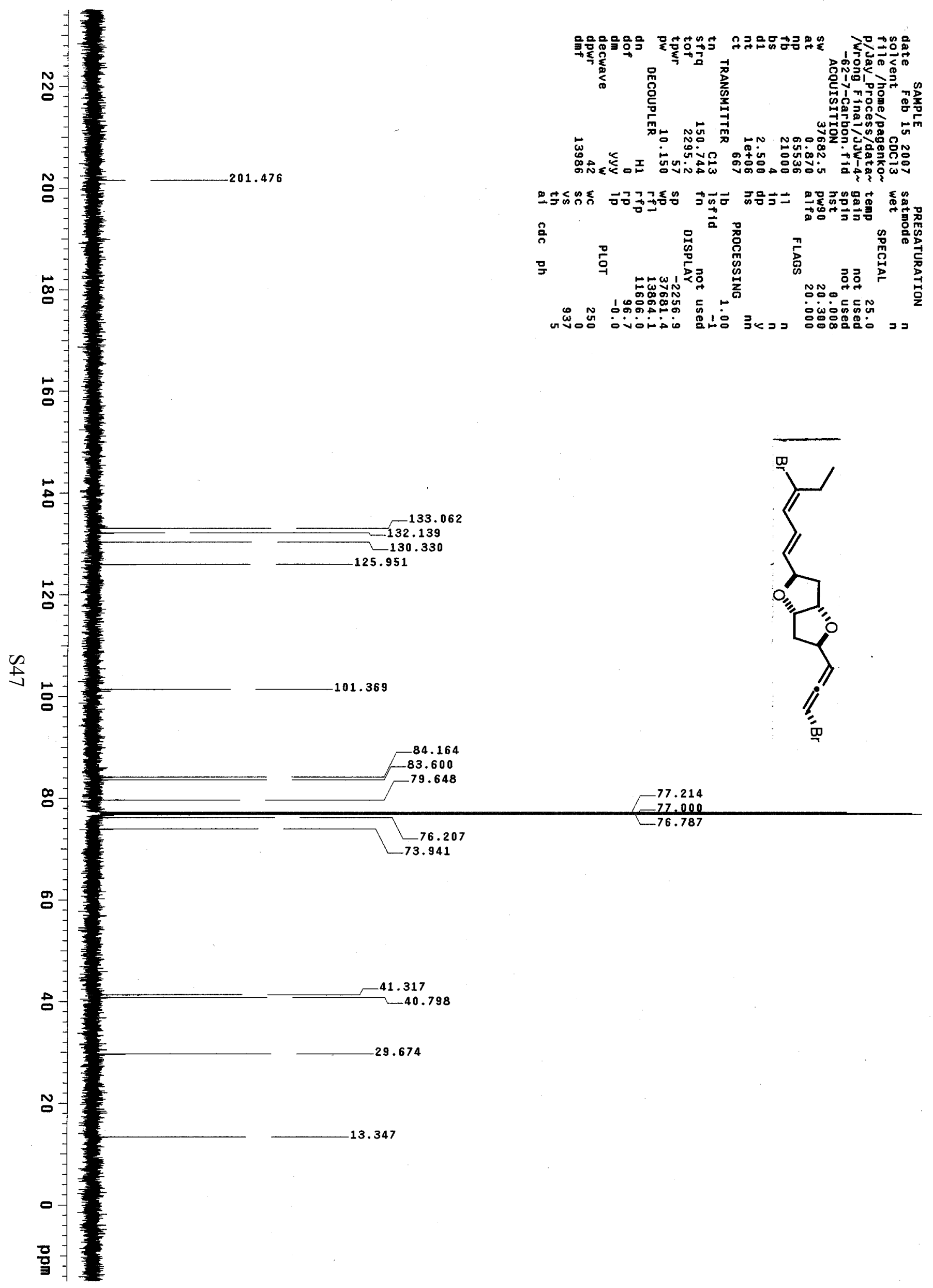

